\title{
Story of an Age-Old Reagent: An Electrophilic Chlorination of Arenes and Heterocycles by 1-Chloro-1,2-benziodoxol-3-one
}

Mengzhou Wang ${ }^{\dagger}$, Yanyan Zhang ${ }^{\dagger}$, Tao Wang $^{\dagger}$, Chao Wang $^{\dagger}$, Dong Xue ${ }^{\dagger}$ and Jianliang Xiao ${ }^{\dagger, \neq}$

† Key Laboratory of Applied Surface and Colloid Chemistry, Ministry of Education, School of Chemistry and Chemical Engineering, Shaanxi Normal University, Xi’an 710062, China

‡ Department of Chemistry, University of Liverpool, Liverpool L69 7ZD, United Kingdom

E-mail: xuedong_welcome@snnu.edu.cn.

\section{Supporting Information}

\section{Table of content}

1. General methods and materials

2. Optimization of reaction solvent 


\section{General methods and materials}

Analytical thin layer chromatography (TLC) was performed on precoated silica gel 60 F254 plates and visualization on TLC was achieved by UV light $(254 \mathrm{~nm})$. Flash column chromatography was undertaken on silica gel (400 mesh) using a proper eluent. ${ }^{1} \mathrm{H}-\mathrm{NMR}$ spectra and ${ }^{13} \mathrm{C}-\mathrm{NMR}$ spectra were recorded at $400 \mathrm{MHz}, 100 \mathrm{MHz}$ on a Bruker Avance 400 spectrometer. All spectra are referenced to $\mathrm{CDCl}_{3}$ residual $\mathrm{CHCl}_{3}$ peak $\left({ }^{1} \mathrm{H}-\mathrm{NMR}=7.26 \mathrm{ppm} ;{ }^{13} \mathrm{C}-\mathrm{NMR}=77.0 \mathrm{ppm}\right)$. All chemical shifts are quoted in parts per million (ppm), measured from the center of the signal except in the case of multiplets of more than one proton, which are quoted as a range. Coupling constants are quoted to the nearest $0.1 \mathrm{~Hz}$. Splitting patterns are abbreviated as follows: singlet (s), doublet (d), triplet (t), quartet (q), dd = doublet of doublet, $\mathrm{td}=$ triplet of doublet, multiplet $(\mathrm{m})$, broad singlet (brs) and combinations thereof.MS data were obtained by using ESI method. Melting points (MP) were determined uncorrected. Dehydrated N, NDimethyformamide was used from a solvent purification system. Other dehydrated solvents were purchased and used as obtained. 1-Chloro-1,2-benziodoxol-3-(1H)-one was prepared by known method. ${ }^{1}$ BODIPY dyes were prepared according to the literature procedure. ${ }^{2}$ All other reagents were used as obtained from commercial source, unless otherwise stated.

\section{Optimization of reaction solvent}

Table S1. Optimization of reaction solvent for chlorination of 1-tosyl- $1 \mathrm{H}$-indole ${ }^{\mathrm{a}}$

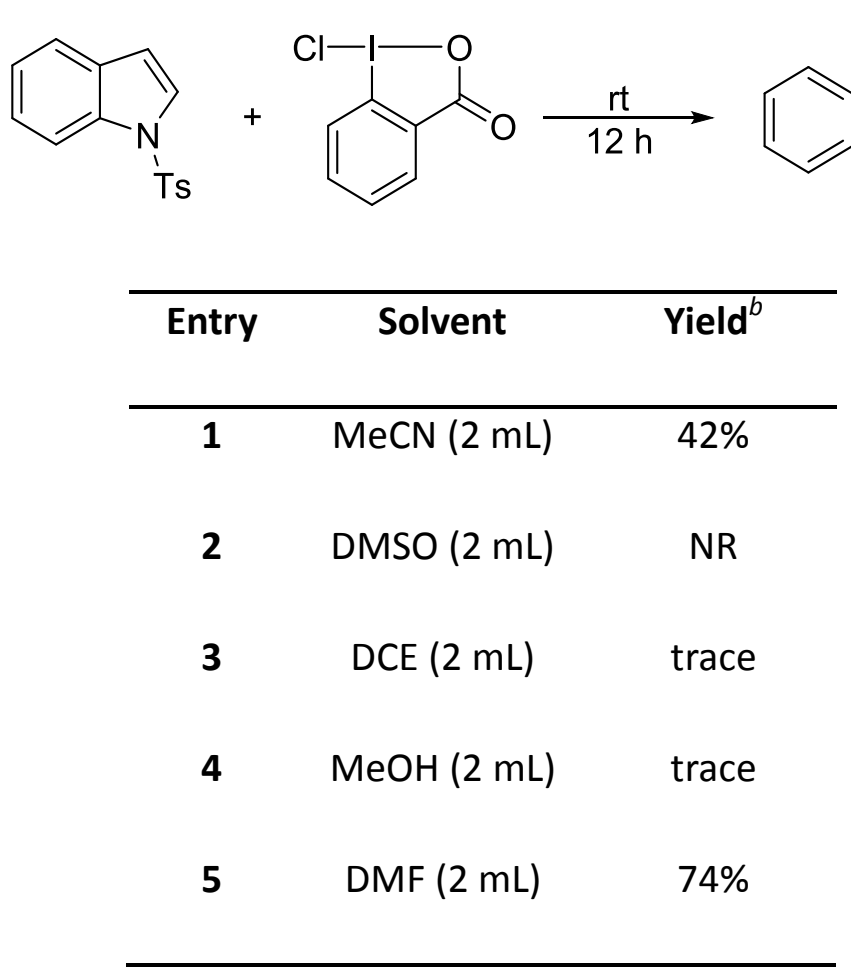


${ }^{a}$ Heterocycle $\left(0.3 \mathrm{mmol}, 1.0\right.$ equiv), chlorination reagent $\left(0.36 \mathrm{mmol}, 1.2\right.$ equiv). ${ }^{b}$ Isolated yields.

\section{Preparation of 1-Chloro-1,2-benziodoxol-3-(1H)-one}<smiles>O=C(O)c1ccccc1I</smiles>

$$
\begin{aligned}
& 0.34 \text { equiv TCICA } \\
& \stackrel{\left(=1.02 \mathrm{Cl}^{+} \text {equiv. }\right)}{\longrightarrow}
\end{aligned}
$$<smiles>O=C1OI(Cl)c2ccccc21</smiles><smiles>O=c1[nH]c(=O)[nH]c(=O)[nH]1</smiles>

Following the reported procedure ${ }^{1}$ a $500 \mathrm{~mL}$ three-necked, round-bottom flask equipped with a magnetic stirring bar, condenser, and dropping funnel was charged under Ar with solid 2-iodobenzoic acid (20 g, $0.0790 \mathrm{~mol}, 1.0$ equiv.), and anhydrous $\mathrm{MeCN}(150 \mathrm{~mL}$ ) was added. The resulting stirred mixture was heated to $75{ }^{\circ} \mathrm{C}$ in an oil bath. The dropping funnel was charged with a solution of trichloroisocyanuric acid $(6.37 \mathrm{~g}, 0.0266 \mathrm{~mol})$ in $30 \mathrm{~mL}$ of anhydrous MeCN. The solution of trichloroisocyanuric acid was dropped into the reaction mixture within $5 \mathrm{~min}$. During the addition of the trichloroisocyanuric acid solution, insoluble isocyanuric acid becomes apparent. The dropping funnel was washed with further anhydrous MeCN (10 mL). After addition was finished, the reaction mixture was refluxed for another $5 \mathrm{~min}$. The reaction mixture was filtered over a funnel with a pad of Celite, and the filter cake was washed with hot MeCN $(20 \mathrm{~mL})$. The combined organic phase was evaporated, and the resulting yellow solid was filtered over a funnel and washed with cold MeCN (10 mL). The mother liquor from filtration was partially concentrated on a rotavapor, and the yellow solid was filtered and washed with cold MeCN, giving the yellow crystals. The combined two crystals were dried $2 \mathrm{~h}$ under high vacuum to give the light yellow crystals $21 \mathrm{~g}$. yield: $95 \%$.

\section{The thermal stability of 1-Chloro-1,2-benziodoxol-3-(1H)-one}

Figure S1 TGA Analysis of 1-Chloro-1,2-benziodoxol-3-one

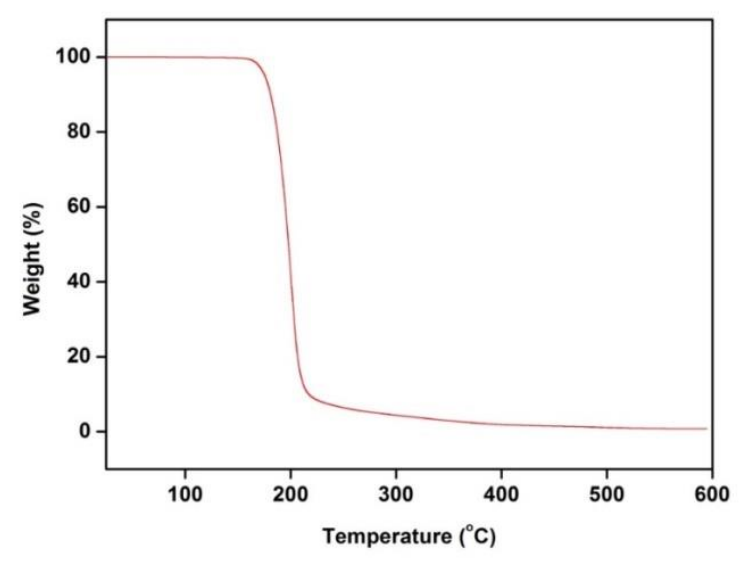




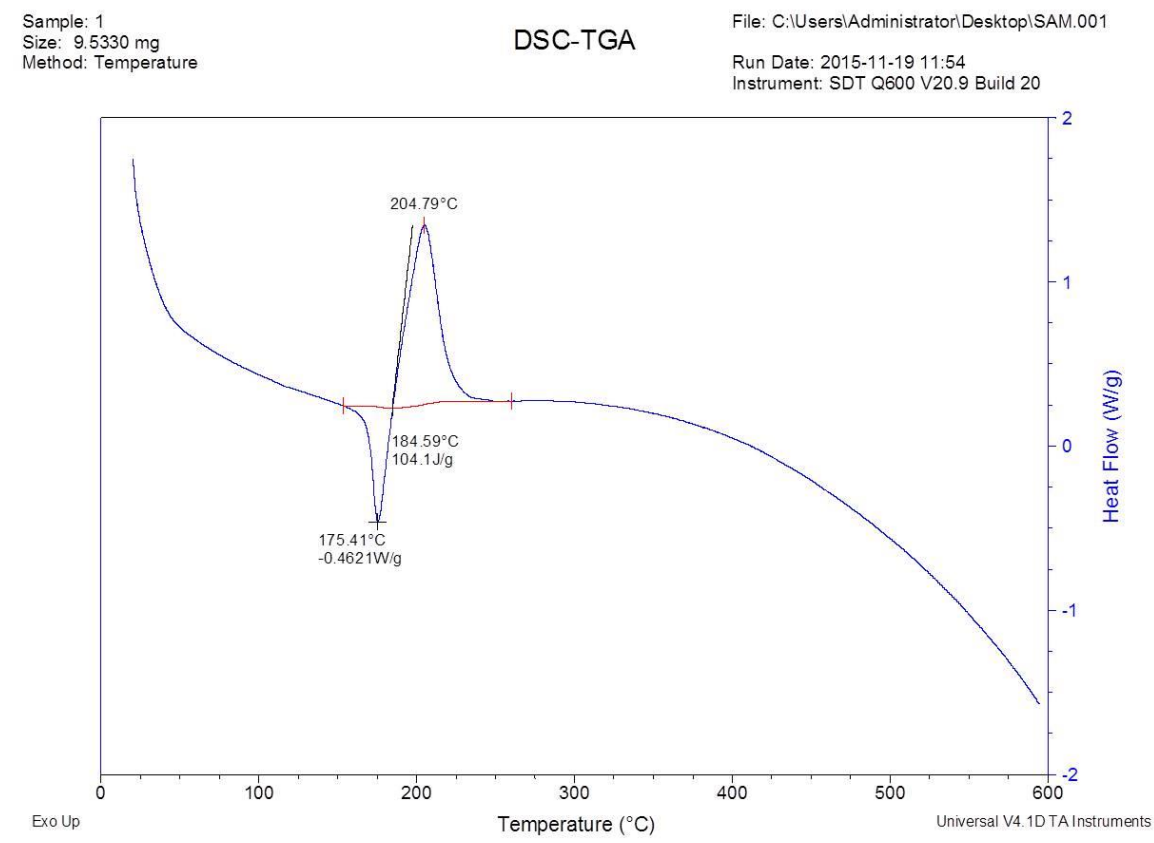

The thermal stability of 1-Chloro-1,2-benziodoxol-3-one by differential scanning calorimetry (DSC) showed no thermal events below $150{ }^{\circ} \mathrm{C}$.

\section{Preparation of BODIPY dyes}

\subsection{Synthesis of 2,8-dichloro-10-ethyl-5,5-difluoro-1,3,7,9-tetramethyl-5H-dipyrrolo[1,2-c:2',1'-}

\section{f][1,3,2]diazaborinin-4-ium-5-uide}<smiles>CCCCCCCC(=O)Cl</smiles>

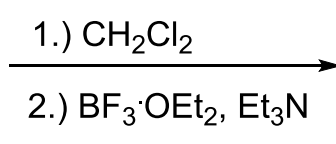<smiles></smiles>

Performed as modification of the established procedure. ${ }^{2}$ To a solution of 2,4-dimethyl-1H-pyrrole (1.35 mL, $13.0 \mathrm{mmol})$ in dry $\mathrm{CH}_{2} \mathrm{Cl}_{2}(10 \mathrm{~mL})$ was added $n$-Octanoyl chloride $(5.18 \mathrm{~mL}, 30.35 \mathrm{mmol})$ dropwise at room temperature over $30 \mathrm{~min}$. The deep red solution was heated to reflux for $1 \mathrm{~h}$. The mixture was poured into $\mathrm{n}$-hexane $(50 \mathrm{~mL})$ after cooling and concentrated to dryness on a rotary evaporator. To a solution of it in dry $\mathrm{CH}_{2} \mathrm{Cl}_{2}(120 \mathrm{~mL})$ was added $\mathrm{NEt}_{3}(5.23 \mathrm{~mL}, 37.5 \mathrm{mmol})$, and the solution was stirred for $10 \mathrm{~min}$ at room temperature. $\mathrm{BF}_{3} \cdot \mathrm{Et}_{2} \mathrm{O}(6.95 \mathrm{~mL}, 56.25 \mathrm{mmol})$ was added dropwise and stirred for $1 \mathrm{~h}$ at room temperature. The deep red solution was washed with saturated aqueous $\mathrm{Na}_{2} \mathrm{CO}_{3}$ solution $\left(4 \times 50 \mathrm{~mL}\right.$ ), dried over $\mathrm{Na}_{2} \mathrm{SO}_{4}$, and concentrated on a rotavapor. The red, oily residue was purified by column chromatography on silica with $n$-pentane $/ \mathrm{CH}_{2} \mathrm{Cl}_{2}=1: 1$. The orange- 
green fluorescing product fraction was dried, and recrystallized from $\mathrm{CH}_{2} \mathrm{Cl}_{2} / \mathrm{MeOH}$ to yield a redorange, crystalline solid.

\subsection{Synthesis of $C^{8}$ BODIPY}
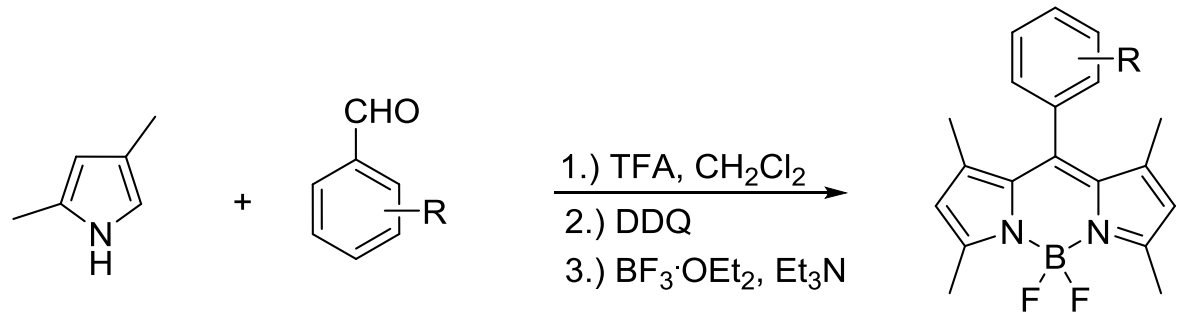

To a solution of benzaldehyde $(2.5 \mathrm{mmol})$ and 2,4-dimethyl- $1 H$-pyrrole $(0.64 \mathrm{~mL}, 6.25 \mathrm{mmol})$ in dry $\mathrm{CH}_{2} \mathrm{Cl}_{2}(125 \mathrm{~mL})$ was added a solution of trifluoroacetic acid $(25 \mu \mathrm{L}, 0.33 \mathrm{mmol})$ in dry $\mathrm{CH}_{2} \mathrm{Cl}_{2}(2.5 \mathrm{~mL})$ slowly at room temperature. 2,3-Dichloro-5,6-Dicyano-1,4-benzoquinone (0.56 g, $2.5 \mathrm{mmol})$ was added after $3 \mathrm{~h}$ stirring under ice bath cooling and stirred for $10 \mathrm{~min}$. The solution was stirred for an additional $1 \mathrm{~h}$ at room temperature. $\mathrm{NEt}_{3}(5 \mathrm{~mL}, 36 \mathrm{mmol})$ was added, followed by slow addition of $\mathrm{BF}_{3} \cdot \mathrm{Et}_{2} \mathrm{O}(5 \mathrm{~mL}, 40.5 \mathrm{mmol})$. After stirring at room temperature for $2 \mathrm{~h}$, the reaction mixture was washed with saturated aqueous $\mathrm{Na}_{2} \mathrm{CO}_{3}$ solution $(3 \times 25 \mathrm{~mL})$, dried over $\mathrm{Na}_{2} \mathrm{SO}_{4}$, and concentrated on a rotary evaporator. The brown, oily residue was purified by column chromatography on silica with npentane/ $\mathrm{CH}_{2} \mathrm{Cl}_{2}=5: 1$, then $2: 1$, then pure $\mathrm{CH}_{2} \mathrm{Cl}_{2}$. The product fraction with greenish fluorescence was concentrated to yield a red-brown solid.

\subsection{Characterization data of BODIPY dyes}

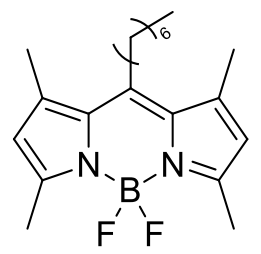

\section{0-ethyl-5,5-difluoro-1,3,7,9-tetramethyl-5H-dipyrrolo[1,2-c:2',1'-f][1,3,2]diazaborinin-4-ium-5-uide}

Red solid, $54 \mathrm{mg}$, yield: 62\%; ${ }^{1} \mathrm{H}$ NMR $\left(400 \mathrm{MHz}, \mathrm{CDCl}_{3}\right) \delta 6.04(\mathrm{~s}, 2 \mathrm{H}), 2.92(\mathrm{t}, J=8.0 \mathrm{~Hz}, 2 \mathrm{H}), 2.51$ (s, $6 \mathrm{H}), 2.40(\mathrm{~s}, 6 \mathrm{H}), 1.61(\mathrm{~m}, 2 \mathrm{H}), 1.48(\mathrm{~m}, 2 \mathrm{H}), 1.33(\mathrm{~m}, 6 \mathrm{H}), 0.89(\mathrm{t}, J=6.8 \mathrm{~Hz}, 3 \mathrm{H}) \mathrm{ppm} ;{ }^{13} \mathrm{C}$ NMR $(100$ $\left.\mathrm{MHz}, \mathrm{CDCl}_{3}\right) \delta 153.6,146.7,140.3,131.4,121.5,31.9,31.7,30.3,29.0,28.5,22.5,16.3,14.4,14.0$ ppm. 


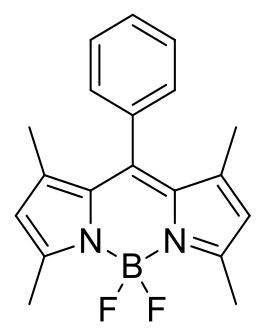

5,5-difluoro-1,3,7,9-tetramethyl-10-phenyl-5H-dipyrrolo[1,2-c:2',1'-f][1,3,2]diazaborinin-4-ium-5uide

Red solid, $56 \mathrm{mg}$, yield: 69\%; ${ }^{1} \mathrm{H}$ NMR $\left(400 \mathrm{MHz}, \mathrm{CDCl}_{3}\right) \delta 7.44(\mathrm{~m}, 3 \mathrm{H}), 7.24(\mathrm{~m}, 2 \mathrm{H}), 5.94(\mathrm{~s}, 2 \mathrm{H}), 2.52$ (s, 6H), $1.34(\mathrm{~s}, 6 \mathrm{H}) \mathrm{ppm} ;{ }^{13} \mathrm{C}$ NMR $\left(100 \mathrm{MHz}, \mathrm{CDCl}_{3}\right) \delta 155.5,143.2,141.8,135.1,131.5,129.2,129.0$, $128.1,121.3,14.7,14.4 \mathrm{ppm}$.

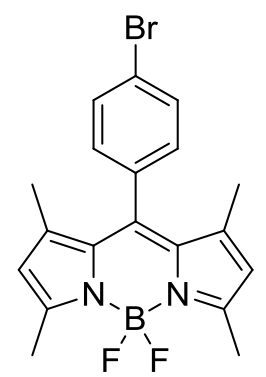

10-(4-bromophenyl)-5,5-difluoro-1,3,7,9-tetramethyl-5H-dipyrrolo[1,2-c:2',1'-f][1,3,2]diazaborinin-4ium-5-uide

Red solid, $72 \mathrm{mg}$, yield: 73\%; ${ }^{1} \mathrm{H}$ NMR $\left(400 \mathrm{MHz}, \mathrm{CDCl}_{3}\right) \delta 7.64(\mathrm{~d}, J=8.3 \mathrm{~Hz}, 2 \mathrm{H}), 7.17(\mathrm{~d}, J=8.3 \mathrm{~Hz}, 2 \mathrm{H})$, $5.98(\mathrm{~s}, 2 \mathrm{H}), 2.55(\mathrm{~s}, 6 \mathrm{H}), 1.41(\mathrm{~s}, 6 \mathrm{H}) \mathrm{ppm} ;{ }^{13} \mathrm{C} \mathrm{NMR}\left(100 \mathrm{MHz}, \mathrm{CDCl}_{3}\right) \delta 155.9,142.9,140.0,133.9$ $132.4,131.1,129.8,123.2,121.4,14.6 \mathrm{ppm}$.<smiles></smiles>

5,5-difluoro-10-(4-methoxyphenyl)-1,3,7,9-tetramethyl-5H-dipyrrolo[1,2-c:2',1'-f][1,3,2]diazaborinin4-ium-5-uide 
Red solid, $67 \mathrm{mg}$, yield: 75\%; ${ }^{1} \mathrm{H}$ NMR $\left(400 \mathrm{MHz}, \mathrm{CDCl}_{3}\right) \delta 7.17$ (d, J=8.5 Hz, 2H), $7.00(\mathrm{~d}, J=8.5 \mathrm{~Hz}, 2 \mathrm{H})$, 5.97 (s, 2H), 3.87 (s, 3H), $2.54(\mathrm{~s}, 6 \mathrm{H}), 1.42(\mathrm{~s}, 6 \mathrm{H}) \mathrm{ppm} ;{ }^{13} \mathrm{C} \mathrm{NMR}\left(100 \mathrm{MHz}, \mathrm{CDCl}_{3}\right) \delta$ 160.1, 155.2, 143.1, $141.8,131.8,129.2,127.0,121.0,114.5,55.3,14.5$ ppm.

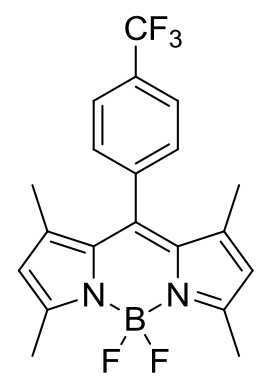

\section{5,5-difluoro-1,3,7,9-tetramethyl-10-(4-(trifluoromethyl)phenyl)-5H-dipyrrolo[1,2-c:2',1'- f][1,3,2]diazaborinin-4-ium-5-uide}

Red solid, $59 \mathrm{mg}$, yield: 60\%; ${ }^{1} \mathrm{H} \mathrm{NMR}\left(400 \mathrm{MHz}, \mathrm{CDCl}_{3}\right) \delta 7.77(\mathrm{~d}, J=8.0 \mathrm{~Hz}, 2 \mathrm{H}), 7.46(\mathrm{~d}, J=8.0 \mathrm{~Hz}, 2 \mathrm{H})$, $6.00(\mathrm{~s}, 2 \mathrm{H}), 2.56(\mathrm{~s}, 6 \mathrm{H}), 1.34(\mathrm{~s}, 6 \mathrm{H}) \mathrm{ppm} ;{ }^{13} \mathrm{C} \mathrm{NMR}\left(100 \mathrm{MHz}, \mathrm{CDCl}_{3}\right) \delta 156.2,142.7,139.5,138.9$, $131.6,131.3,130.9,128.8,126.1$ (q, $J=14.6 \mathrm{~Hz}), 125.1,122.4,121.6,14.4$ ppm.

\section{General procedure of chlorination}

\subsection{Standard procedure of chlorination of heterocycles}

To a stirred solution of heterocycle $(0.3 \mathrm{mmol}, 1.0$ equiv.) in anhydrous DMF $(2 \mathrm{~mL})$ at room temperature was added chlorinating reagent $(0.36 \mathrm{mmol}, 1.2$ equiv. $)$ and the mixture was stirred for $12 \mathrm{~h}$ under air condition. After completion of reaction monitored by TLC, the resulting mixture quenched with saturated aqueous $\mathrm{NaHCO}_{3}$, then extracted with ethyl acetate $(15 \mathrm{~mL} \times 3)$. The combined organic layer was dried over anhydrous $\mathrm{Na}_{2} \mathrm{SO}_{4}$ and filtered. The solution was removed by evaporation under reduced pressure to afford the crude product. The crude product was then purified by column chromatography on silica gel using petroleum ether/ethyl acetate as eluent to give the desired product.

\subsection{Chlorination of BODIPY dyes standard procedure}

To a stirred solution of BODIPY $(0.3 \mathrm{mmol}, 1.0$ equiv) in anhydrous DMF $(2 \mathrm{~mL})$ at room temperature was added chlorinating reagent $(0.66 \mathrm{mmol}, 2.2$ equiv) and the mixture was stirred for $12 \mathrm{~h}$ under air condition. After completion of reaction as indicated by TLC, the resulting mixture quenched with saturated aqueous $\mathrm{NaHCO}_{3}$, then extracted with ethyl acetate $(15 \mathrm{~mL} \times 3)$. The combined organic layer was dried over anhydrous $\mathrm{Na}_{2} \mathrm{SO}_{4}$ and filtered. The solution was removed by evaporation under reduced 
pressure to afford the crude product. The crude product was then purified by column chromatography on silica gel using petroleum ether/ethyl acetate as eluent to give the desired product.

\subsection{Procedure of chlorination of heterocycles at gram-scale}

To a solution of heterocycle $(1 \mathrm{~g}, 1.0$ equiv) in DMF $(0.1 \mathrm{M})$ at room temperature was added the chlorination reagent (1.2 equiv) with vigorously stirring. Reaction was monitored by TLC, the result mixture quenched with saturated aqueous $\mathrm{NaHCO}_{3}$, then extracted with ethyl acetate three times. The combined organic layer was dried over anhydrous $\mathrm{Na}_{2} \mathrm{SO}_{4}$, filtered, and the solution was removed by evaporation under reduced pressure to afford the product.

\subsection{Procedure of chlorination Aripiprazole}

To a solution of Aripiprazole $\left(1 \mathrm{~g}, 1.0\right.$ equiv) in DMF $(10 \mathrm{~mL})$ at $-50{ }^{\circ} \mathrm{C}$ was added the chlorination reagent (1.86 g, 3.0 equiv) with vigorously stirring. TLC monitored the reaction until completion. After $12 \mathrm{~h}$, the result mixture quenched with saturated aqueous $\mathrm{NaHCO}_{3}$, then extracted with ethyl acetate three times. The combined organic layer was dried over anhydrous $\mathrm{Na}_{2} \mathrm{SO}_{4}$, filtered, and then the solution was removed by evaporation under reduced pressure to afford the product.

\section{Radical trapping experiments}

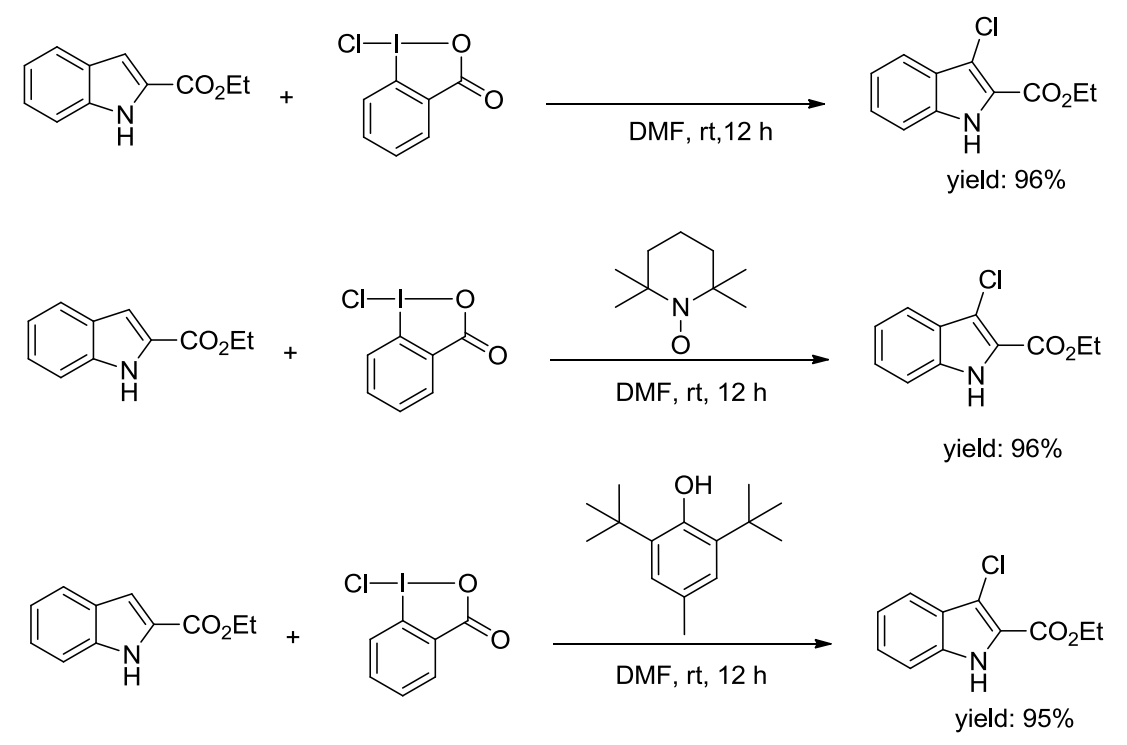

To a stirred solution of ethyl $1 \mathrm{H}$-indole-2-carboxylate $(0.3 \mathrm{mmol}, 1.0$ equiv) in anhydrous DMF (2 mL) at room temperature was added chlorinating reagent $(0.36 \mathrm{mmol}, 1.2$ equiv), radical scavenger (0.36 mmol, 1.2 equiv) and the mixture was stirred for $12 \mathrm{~h}$ under air condition. After completion of reaction monitored by TLC, the resulting mixture quenched with saturated aqueous $\mathrm{NaHCO}_{3}$, then extracted 
with ethyl acetate $(15 \mathrm{~mL} \times 3)$. The combined organic layer was dried over anhydrous $\mathrm{Na}_{2} \mathrm{SO}_{4}$ and filtered. The solution was removed by evaporation under reduced pressure to afford the crude product. The crude product was then purified by column chromatography on silica gel using petroleum ether/ethyl acetate as eluent to give the desired product.

\section{Characterization data}

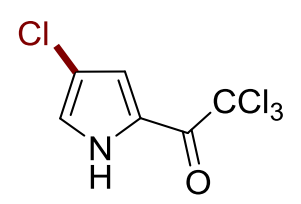

\section{(2) 2,2,2-trichloro-1-(4-chloro-1H-pyrrol-2-yl)ethanone}

Known compound. ${ }^{3}$ White solid, $55.1 \mathrm{mg}$, m.p. 119-121 ${ }^{\circ} \mathrm{C} ;{ }^{1} \mathrm{H}$ NMR $\left(400 \mathrm{MHz}, \mathrm{CDCl}_{3}\right) \delta 9.47$ (brs, $\left.1 \mathrm{H}\right)$, $7.27(\mathrm{dd}, J=2.4,1.2 \mathrm{~Hz}, 1 \mathrm{H}), 7.11(\mathrm{dd}, J=3.2,1.2 \mathrm{~Hz}, 1 \mathrm{H}) \mathrm{ppm} ;{ }^{13} \mathrm{C} \mathrm{NMR}\left(100 \mathrm{MHz}, \mathrm{CDCl}_{3}\right) \delta 172.8$, $124.2,122.0,119.5,115.4,94.3$ ppm.

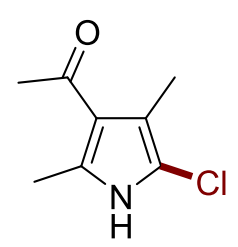

\section{(3) 1-(5-chloro-2,4-dimethyl-1H-pyrrol-3-yl)ethan-1-one}

White solid, 35.9 mg, m.p. $132-134{ }^{\circ} \mathrm{C} ;{ }^{1} \mathrm{H}$ NMR (400 MHz, CDCl $) \delta 11.66$ (brs, 1H), $2.31(\mathrm{~s}, 3 \mathrm{H}$ ), 2.24 (s, 3H), 2.03 (s, 3H) ppm; $\left.{ }^{13} \mathrm{C} \mathrm{NMR} \mathrm{(100} \mathrm{MHz,} \mathrm{CDCl}_{3}\right) \delta 195.1,135.6,122.3,116.3,112.2,32.5,16.1,13.1$ ppm. HRMS (ESI) m/z calcd. for $\mathrm{C}_{8} \mathrm{H}_{10} \mathrm{CINNaO}[\mathrm{M}+\mathrm{Na}]^{+}:$194.0343, found: 194.0345 .

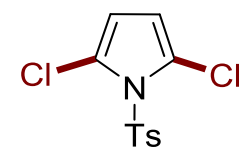

\section{(4) 2,5-dichloro-1-tosyl-1H-pyrrole}

Known compound. ${ }^{3}$ White solid, $57.2 \mathrm{mg}$, m.p. 76-78 ${ }^{\circ} \mathrm{C} ;{ }^{1} \mathrm{H} \mathrm{NMR}(400 \mathrm{MHz}, \mathrm{CDCl} 3) \delta 7.89$ (dd, J = 6.4, $1.6 \mathrm{~Hz}, 2 \mathrm{H}), 7.34(\mathrm{~d}, \mathrm{~J}=8.0 \mathrm{~Hz}, 2 \mathrm{~Hz}), 6.10(\mathrm{~s}, 2 \mathrm{H}), 2.43(\mathrm{~s}, 3 \mathrm{H}) \mathrm{ppm} ;{ }^{13} \mathrm{C} \mathrm{NMR}(100 \mathrm{MHz}, \mathrm{CDCl} 3) \delta 145.8$, $135.4,130.0,127.6,117.6,112.6,21.7$ ppm. 


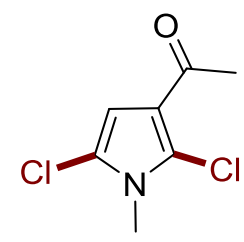

\section{(5) 1-(2, 5-dichloro-1-methyl-1H-pyrrol-3-yl)ethanone}

Known compound. ${ }^{3}$ Yellow oil, $40.1 \mathrm{mg},{ }^{1} \mathrm{H}$ NMR (400 MHz, $\left.\mathrm{CDCl}_{3}\right) \delta 6.54(\mathrm{~s}, 1 \mathrm{H}), 3.58(\mathrm{~s}, 3 \mathrm{H}), 2.45$ (s, 3H) ppm; ${ }^{13} \mathrm{C}$ NMR $\left(100 \mathrm{MHz}, \mathrm{CDCl}_{3}\right) \delta 191.3,120.7,119.5,117.1,107.8,31.3,28.8$ ppm.<smiles>Nc1nc(Cl)cs1</smiles>

\section{(6) 4-chlorothiazol-2-amine}

Yellow solid, $27.4 \mathrm{mg} ;{ }^{1} \mathrm{H}$ NMR $\left(400 \mathrm{MHz}, \mathrm{CDCl}_{3}\right) \delta 6.89(\mathrm{~s}, 1 \mathrm{H}), 4.93(\mathrm{brs}, 2 \mathrm{H}) \mathrm{ppm} ;{ }^{13} \mathrm{C} \mathrm{NMR}(100 \mathrm{MHz}$, DMSO-d $\left.{ }_{6}\right) \delta 168.9,138.5,110.9$ ppm. HRMS (ESI) m/z calcd. for $\mathrm{C}_{3} \mathrm{H}_{4} \mathrm{CIN}_{2} \mathrm{~S}[\mathrm{M}+\mathrm{H}]^{+}:$: 134.9778, found: 134.9781.

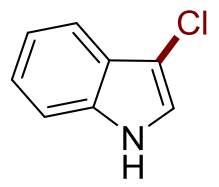

\section{(7) 3-chloro-1H-indolex}

Known compound. ${ }^{4}$ Yellow solid, $29.5 \mathrm{mg}$, m.p. 76-77 ${ }^{\circ} \mathrm{C} ;{ }^{1} \mathrm{H} \mathrm{NMR}\left(400 \mathrm{MHz}, \mathrm{CDCl}_{3}\right) \delta 7.46(\mathrm{~d}, \mathrm{~J}=7.8 \mathrm{~Hz}$, $1 \mathrm{H}), 7,37(\mathrm{~d}, J=8.0 \mathrm{~Hz}, 1 \mathrm{H}), 7.27-7.18(\mathrm{~m}, 3 \mathrm{H}) \mathrm{ppm} ;{ }^{13} \mathrm{C} \mathrm{NMR}\left(100 \mathrm{MHz}, \mathrm{CDCl}_{3}\right) \delta 134.9,125.4,123.1$, 120.7, 120.4, 118.2, 111.4, 106.6 ppm.

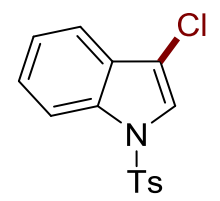

(8) 3-chloro-1-tosyl-1H-indole

Known compound. ${ }^{3}$ White solid, $67.6 \mathrm{mg}$, m.p. $110-111{ }^{\circ} \mathrm{C} ;{ }^{1} \mathrm{H}$ NMR $\left(400 \mathrm{MHz}, \mathrm{CDCl}_{3}\right) \delta 8.01(\mathrm{~d}, J=8.4$ $\mathrm{Hz}, 1 \mathrm{H}), 7.76(\mathrm{~d}, J=8.4 \mathrm{~Hz}, 2 \mathrm{H}), 7.54(\mathrm{~d}, J=8.8 \mathrm{~Hz}, 2 \mathrm{H}), 7.38(\mathrm{t}, J=7.2 \mathrm{~Hz}, 1 \mathrm{H}), 7.30(\mathrm{t}, J=7.2 \mathrm{~Hz}, 1 \mathrm{H})$, $7.23(\mathrm{~d}, J=8.4 \mathrm{~Hz}, 1 \mathrm{H}), 2.34$ (s, 3H) ppm; ${ }^{13} \mathrm{C} \mathrm{NMR}\left(100 \mathrm{MHz}, \mathrm{CDCl}_{3}\right) \delta$ 145.3, 134.8, 134.1, 130.0, 128.4, 126.9, 125.8, 123.8, 122.3, 119.0, 113.8, 113.7, 21.5 ppm. 


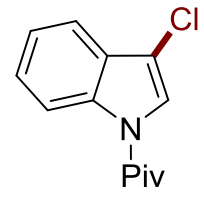

(9) 1-(3-chloro-1H-indol-1-yl)-2,2-dimethylpropan-1-one

Known compound. ${ }^{3}$ White solid, $62.1 \mathrm{mg}$, m.p. $80-82{ }^{\circ} \mathrm{C} ;{ }^{1} \mathrm{H}$ NMR $\left(400 \mathrm{MHz}, \mathrm{CDCl}_{3}\right) \delta 8.52(\mathrm{~d}, J=8.0 \mathrm{~Hz}$, 1H), $7.73(\mathrm{~s}, 1 \mathrm{H}), 7.58(\mathrm{q}, J=7.2 \mathrm{~Hz}, 1 \mathrm{H}), 7.43(\mathrm{~m}, 1 \mathrm{H}), 7.34(\mathrm{~m}, 1 \mathrm{H}), 1.52(\mathrm{~s}, 1 \mathrm{H}) \mathrm{ppm} ;{ }^{13} \mathrm{C}$ NMR $(100$ $\left.\mathrm{MHz}, \mathrm{CDCl}_{3}\right) \delta 176.4,135.9,127.0,126.3,124.0,121.9,118.0,117.4,113.0,41.24,28.62 \mathrm{ppm}$.

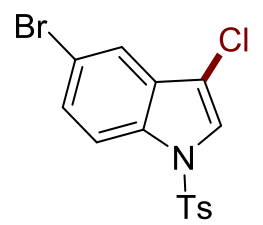

(10) 5-bromo-3-chloro-1-tosyl-1H-indole

White solid, $89.6 \mathrm{mg}$, m.p. $108-110{ }^{\circ} \mathrm{C} ;{ }^{1} \mathrm{H}$ NMR $\left(400 \mathrm{MHz}, \mathrm{CDCl}_{3}\right) \delta 7.86(\mathrm{~d}, J=8.8 \mathrm{~Hz}, 1 \mathrm{H}), 7.73(\mathrm{~d}, J=$ $8.4 \mathrm{~Hz}, 2 \mathrm{H}), 7.65(\mathrm{~d}, J=1.6 \mathrm{~Hz}, 1 \mathrm{H}), 7.56(\mathrm{~d}, J=3.6 \mathrm{~Hz}, 1 \mathrm{H}), 7.39$ (dd, $J=15.6,1.6 \mathrm{~Hz}, 1 \mathrm{H}), 7.22(\mathrm{~d}, J=$ $8.0 \mathrm{~Hz}, 2 \mathrm{H}), 6.58(\mathrm{t}, J=3.2 \mathrm{~Hz}, 1 \mathrm{H}), 2.34(\mathrm{~s}, 3 \mathrm{H}) \mathrm{ppm} ;{ }^{13} \mathrm{C} \mathrm{NMR}\left(100 \mathrm{MHz}, \mathrm{CDCl}_{3}\right) \delta 146.1,133.3,132.1$, 130.4, 129.3, 128.8, 126.8, 124.9, 121.0, 116.9, 115.5, 111.4, 21.0 ppm. HRMS (ESI) $\mathrm{m} / \mathrm{z}$ calcd. for $\mathrm{C}_{15} \mathrm{H}_{11} \mathrm{BrClNNaO} \mathrm{S}_{2}[\mathrm{M}+\mathrm{Na}]^{+}: 405.9274$, found: 405.9278 .

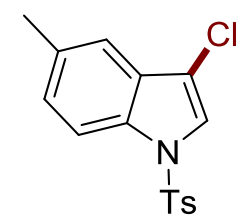

(11) 3-chloro-5-methyl-1-tosyl-1H-indole

Yellow solid, 73.7 mg, m.p. $100-102{ }^{\circ} \mathrm{C} ;{ }^{1} \mathrm{H}$ NMR $\left(400 \mathrm{MHz}, \mathrm{CDCl}_{3}\right) \delta 8.13(\mathrm{~d}, J=8.4 \mathrm{~Hz}, 1 \mathrm{H}), 7.73$ (d, $J=$ $7.2 \mathrm{~Hz}, 2 \mathrm{H}), 7.24(\mathrm{~m}, 5 \mathrm{H}), 2.44(\mathrm{~s}, 3 \mathrm{H}), 2.36(\mathrm{~s}, 3 \mathrm{H}) \mathrm{ppm} ;{ }^{13} \mathrm{C} \mathrm{NMR}\left(100 \mathrm{MHz}, \mathrm{CDCl}_{3}\right) \delta 146.4,134.8$, $133.3,132.3,130.4,128.0,126.6,125.9,120.8,117.1,114.7,112.8,21.0,20.6$ ppm. HRMS (ESI) m/z calcd. for $\mathrm{C}_{16} \mathrm{H}_{15} \mathrm{ClNO}_{2} \mathrm{~S}[\mathrm{M}+\mathrm{H}]^{+}: 320.0506$, found: 320.0509 .

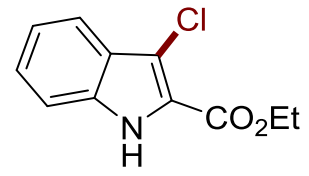

(12) Ethyl 3-chloro-1H-indole-2-carboxylate 
Known compound. ${ }^{3}$ Yellow solid, $64.2 \mathrm{mg}$, m.p. $123-124{ }^{\circ} \mathrm{C} ;{ }^{1} \mathrm{H}$ NMR $\left(400 \mathrm{MHz}, \mathrm{CDCl}_{3}\right) \delta 8.90(\mathrm{brs}, 1 \mathrm{H})$, $7.72(\mathrm{~d}, J=8 \mathrm{~Hz}, 1 \mathrm{H}), 7.38(\mathrm{~m}, 2 \mathrm{H}), 7.22(\mathrm{~m}, 1 \mathrm{H}), 4.46(\mathrm{q}, J=7.2 \mathrm{~Hz}, 2 \mathrm{H}), 1.45(\mathrm{t}, J=7.2 \mathrm{~Hz}, 3 \mathrm{H}) \mathrm{ppm} ;{ }^{13} \mathrm{C}$ NMR $\left(100 \mathrm{MHz}, \mathrm{CDCl}_{3}\right) \delta 160.9,134.6,126.5,126.2,122.4,121.3,120.2,112.4,112.0,61.3,14.3$ ppm.

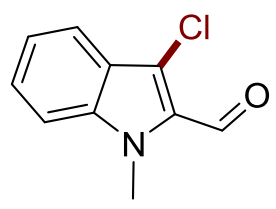

\section{(13) 3-chloro-1-methyl-1H-indole-2-carbaldehyde}

Known compound. ${ }^{3}$ White solid, $57.3 \mathrm{mg}$, m.p. $83-84{ }^{\circ} \mathrm{C} ;{ }^{1} \mathrm{H}$ NMR $\left(400 \mathrm{MHz}, \mathrm{CDCl}_{3}\right) \delta 10.18(\mathrm{~s}, 1 \mathrm{H}), 7.74$ $(\mathrm{d}, J=8.4 \mathrm{~Hz}, 1 \mathrm{H}), 7.46(\mathrm{t}, J=6.8 \mathrm{~Hz}, 1 \mathrm{H}), 7.37(\mathrm{~d}, J=8.4 \mathrm{~Hz}, 1 \mathrm{H}), 7.23(\mathrm{t}, J=8.0 \mathrm{~Hz}, 1 \mathrm{H}), 4.07(\mathrm{~s}, 3 \mathrm{H})$ ppm; ${ }^{13} \mathrm{C}$ NMR $\left(100 \mathrm{MHz}, \mathrm{CDCl}_{3}\right) \delta 181.3,138.6,129.1,128.1,124.2,121.4,120.5,119.2,110.4,31.7$ ppm.

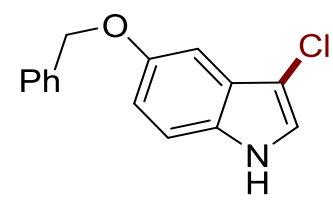

(14) 5-(benzyloxy)-3-chloro-1H-indole

White solid, $64.1 \mathrm{mg}$, m.p. $87-88{ }^{\circ} \mathrm{C} ;{ }^{1} \mathrm{H}$ NMR $\left(400 \mathrm{MHz}, \mathrm{CDCl}_{3}\right) \delta 7.96$ (brs, $\left.1 \mathrm{H}\right), 7.48(\mathrm{~d}, \mathrm{~J}=7.2 \mathrm{~Hz}, 2 \mathrm{H})$, $7.39(\mathrm{t}, J=7.6 \mathrm{~Hz}, 2 \mathrm{H}), 7.32(\mathrm{t}, J=7.2 \mathrm{~Hz}, 1 \mathrm{H}), 7.24(\mathrm{~s}, 1 \mathrm{H}), 7.14(\mathrm{dd}, J=6.8,2.0 \mathrm{~Hz}, 2 \mathrm{H}), 6.97(\mathrm{dd}, J=8.8$, $2.4 \mathrm{~Hz}, 1 \mathrm{H}), 5.12(\mathrm{~s}, 2 \mathrm{H}) \mathrm{ppm} ;{ }^{13} \mathrm{C} \mathrm{NMR}\left(100 \mathrm{MHz}, \mathrm{CDCl}_{3}\right) \delta$ 153.9, 137.3, 130.1, 128.5, 127.9, 127.6, 125.7, 121.4, 114.5, 112.4, 106.1, 100.870 .7 ppm. HRMS (ESI) $\mathrm{m} / \mathrm{z}$ calcd. for $\mathrm{C}_{15} \mathrm{H}_{12} \mathrm{CINNaO}[\mathrm{M}+\mathrm{Na}]^{+}$: 280.0499, found: 280.0503 .

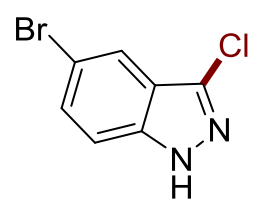

\section{(15) 5-bromo-3-chloro-1H-indazole}

White solid, 59.4 mg, m.p. $168-170{ }^{\circ} \mathrm{C} ;{ }^{1} \mathrm{H}$ NMR $(400 \mathrm{MHz}$, DMSO-d 6 ) $\delta 7.88(\mathrm{~s}, 1 \mathrm{H}), 7.57$ (d, $J=8.0 \mathrm{~Hz}$, 2H) ppm; ${ }^{13} \mathrm{C}$ NMR $\left(100 \mathrm{MHz}\right.$, DMSO-d $\left.\mathrm{d}_{6}\right) \delta 139.8,131.4,130.3,120.9,120.8,113.6,113.1 \mathrm{ppm}$. HRMS $\left(\mathrm{ESI}^{+}\right) \mathrm{m} / \mathrm{z}$ calcd. for $\mathrm{C}_{7} \mathrm{H}_{5} \mathrm{ClBrN}_{2}[\mathrm{M}+\mathrm{H}]^{+}: 230.9319$, found: 230.9330 . 


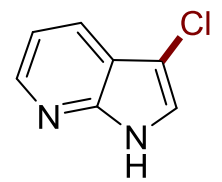

(16) 3-chloro-1H-pyrrolo[2,3-b]pyridine

Yellow solid, $35.1 \mathrm{mg}$, m.p. $105-107^{\circ} \mathrm{C} ;{ }^{1} \mathrm{H}$ NMR (400 MHz, $\left.\mathrm{CDCl}_{3}\right) \delta 11.40$ (brs, $\left.1 \mathrm{H}\right), 8.37(\mathrm{~d}, J=4.0 \mathrm{~Hz}$, $1 \mathrm{H}), 8.00(\mathrm{dd}, J=8.0,1.2 \mathrm{~Hz}, 1 \mathrm{H}), 7.36(\mathrm{~s}, 1 \mathrm{H}), 7.18(\mathrm{dd}, J=8.0,4.8 \mathrm{~Hz}, 1 \mathrm{H}) \mathrm{ppm} ;{ }^{13} \mathrm{C} \mathrm{NMR}(100 \mathrm{MHz}$, $\left.\mathrm{CDCl}_{3}\right) \delta 147.1,143.5,127.1,121.9,118.5,116.3,104.4 \mathrm{ppm}$. HRMS (ESI) $\mathrm{m} / z$ calcd. for $\mathrm{C}_{7} \mathrm{H}_{6} \mathrm{CIN}_{2}$ $[\mathrm{M}+\mathrm{H}]^{+}:$153.0214, found: 153.0215 .<smiles>Clc1ccc2c(Cl)c[nH]c2n1</smiles>

(17) 3,6-dichloro-1H-pyrrolo[2,3-b]pyridine

White solid, $45.2 \mathrm{mg} ;{ }^{1} \mathrm{H}$ NMR (400 MHz, DMSO-d 6 ) $\delta 12.22$ (brs, 1H), 7.99 (d, J = 8.0 Hz, 1H), 7.73 (d, J $=2.8 \mathrm{~Hz}, 1 \mathrm{H}), 7.23(\mathrm{~d}, J=8.4 \mathrm{~Hz}, 1 \mathrm{H}) \mathrm{ppm} ;{ }^{13} \mathrm{C}$ NMR $\left(100 \mathrm{MHz}, \mathrm{DMSO}-\mathrm{d}_{6}\right) \delta 145.3,144.2,128.9,123.9$, 116.1, 102.3 ppm. HRMS $\left(E S I^{+}\right) \mathrm{m} / z$ calcd. for $\mathrm{C}_{7} \mathrm{H}_{5} \mathrm{Cl}_{2} \mathrm{~N}_{2}[\mathrm{M}+\mathrm{H}]^{+}:$186.9824, found: 186.9828 .

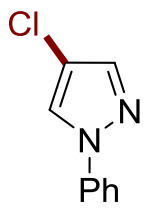

(18) 4-chloro-1-phenyl-1H-pyrazole

Known compound. ${ }^{3}$ White solid, $52.9 \mathrm{mg}$, m.p. 66-68 ${ }^{\circ} \mathrm{C} ;{ }^{1} \mathrm{H}$ NMR $\left(400 \mathrm{MHz}, \mathrm{CDCl}_{3}\right) \delta 7.90(\mathrm{~s}, 1 \mathrm{H}), 7.63$ $(\mathrm{dd}, J=8.8,1.6 \mathrm{~Hz}, 3 \mathrm{H}), 7.46(\mathrm{t}, J=7.2 \mathrm{~Hz}, 2 \mathrm{H}), 7.31(\mathrm{t}, J=7.6 \mathrm{~Hz}, 1 \mathrm{H}) \mathrm{ppm} ;{ }^{13} \mathrm{C} \mathrm{NMR}\left(100 \mathrm{MHz}, \mathrm{CDCl}_{3}\right) \delta$ $139.7,139.4,129.5,127.0,124.8,118.9,112.4$ ppm.

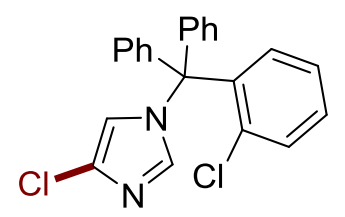

(19) 4-chloro-1-((2-chlorophenyl)diphenylmethyl)-1H-imidazole 
Known compound. ${ }^{3}$ White solid, $68.1 \mathrm{mg}$, m.p.168-170 ${ }^{\circ} \mathrm{C} ;{ }^{1} \mathrm{H}$ NMR $\left(400 \mathrm{MHz}, \mathrm{CDCl}_{3}\right) \delta$ 7.44-7.43 (m, $1 \mathrm{H}), 7.42-7.34(\mathrm{~m}, 7 \mathrm{H}), 7.30-7.27(\mathrm{~m}, 2 \mathrm{H}), 7.18-7.16(\mathrm{~m}, 3 \mathrm{H}), 6.94(\mathrm{dd}, J=8.0,1.5 \mathrm{~Hz}, 1 \mathrm{H}), 6.65(\mathrm{~d}, J=$ $1.6 \mathrm{~Hz}, 1 \mathrm{H}) \mathrm{ppm} ;{ }^{13} \mathrm{C} \mathrm{NMR}\left(100 \mathrm{MHz}, \mathrm{CDCl}_{3}\right) \delta 140.1,139.6,137.5,135.5,132.3,130.3,130.1,129.1$, 128.4, 128.1, 127.1, 117.3, 76.2 ppm. HRMS (ESI ${ }^{+}$) m/z calcd. for $\mathrm{C}_{22} \mathrm{H}_{16} \mathrm{Cl}_{2} \mathrm{~N}_{2} \mathrm{Na}[\mathrm{M}+\mathrm{Na}]^{+}:$401.0582, found: 401.0586 .<smiles>Clc1nc(Cl)c2c(Cl)c[nH]c2n1</smiles>

\section{(20) 2,4,5-trichloro-7H-pyrrolo[2,3-d]pyrimidine}

Known compound. ${ }^{3}$ White solid, 53.7 mg, m.p. 180-182 ${ }^{\circ} \mathrm{C} ;{ }^{1} \mathrm{H}$ NMR (400 MHz, DMSO-d 6 ) $\delta 13.08$ (brs, 1H), $7.96(\mathrm{~s}, 1 \mathrm{H}) \mathrm{ppm} ;{ }^{13} \mathrm{C}$ NMR $\left(100 \mathrm{MHz}, \mathrm{DMSO}-\mathrm{d}_{6}\right) \delta 151.5,150.8,150.5,126.9,111.9,102.1 \mathrm{ppm}$.<smiles>CCOC(OCC)c1nc2ncccn2c1Cl</smiles>

\section{(21) ethyl 3-methylimidazo[1,2-a]pyrimidine-2-carboxylate}

Known compound. ${ }^{3}$ White solid, $48.6 \mathrm{mg}$, m.p. $165-166{ }^{\circ} \mathrm{C} ;{ }^{1} \mathrm{H} \mathrm{NMR}\left(400 \mathrm{MHz}, \mathrm{CDCl}_{3}\right) \delta 8.68(\mathrm{q}, \mathrm{J}=4 \mathrm{~Hz}$, $1 \mathrm{H}), 8.47(\mathrm{q}, J=6.8 \mathrm{~Hz}, 1 \mathrm{H}), 6.97(\mathrm{q}, J=6.8 \mathrm{~Hz}, 1 \mathrm{H}), 4.47(\mathrm{q}, J=7.2 \mathrm{~Hz}, 2 \mathrm{H}), 1.44(\mathrm{t}, J=7.2 \mathrm{~Hz}, 3 \mathrm{H}) \mathrm{ppm}$; ${ }^{13} \mathrm{CNMR}\left(100 \mathrm{MHz}, \mathrm{CDCl}_{3}\right) \delta 161.7,152.4,145.7,132.3,131.1,113.0,110.4,61.6,14.3 \mathrm{ppm}$.<smiles>CN(C)c1c(Cl)cncc1Cl</smiles>

\section{(22) 3,5-dichloro-N,N-dimethylpyridin-4-amine}

Black oil, $31.4 \mathrm{mg} ;{ }^{1} \mathrm{H}$ NMR $\left(400 \mathrm{MHz}, \mathrm{CDCl}_{3}\right) \delta 8.30(\mathrm{~s}, 2 \mathrm{H}), 3.01(\mathrm{~s}, 6 \mathrm{H}) \mathrm{ppm} ;{ }^{13} \mathrm{C} \mathrm{NMR}\left(100 \mathrm{MHz}, \mathrm{CDCl}_{3}\right)$ $\delta 152.5,149.0,128.2,42.6$ ppm. HRMS $\left(\mathrm{ESI}^{+}\right) \mathrm{m} / z$ calcd. for $\mathrm{C}_{7} \mathrm{H}_{9} \mathrm{Cl}_{2} \mathrm{~N}_{2}[\mathrm{M}+\mathrm{H}]^{+}:$191.0137, found: 191.0141.<smiles>Nc1ccc(Cl)nc1Cl</smiles> 
Yellow solid, $24.3 \mathrm{mg}$, m.p. 100-102 ${ }^{\circ} \mathrm{C} ;{ }^{1} \mathrm{H}$ NMR (400 MHz, CDCl $) \delta 7.06(\mathrm{~d}, J=8 \mathrm{~Hz}, 1 \mathrm{H}), 7.01(\mathrm{~d}, J=8$ $\mathrm{Hz}, 1 \mathrm{H}), 4.09$ (brs, 1H) ppm; ${ }^{13} \mathrm{C}$ NMR $\left(100 \mathrm{MHz}, \mathrm{CDCl}_{3}\right) \delta 140.1,139.0,136.3,126.4,125.0$ ppm. HRMS $\left(\mathrm{ESI}^{+}\right) \mathrm{m} / z$ calcd. for $\mathrm{C}_{5} \mathrm{H}_{4} \mathrm{Cl}_{2} \mathrm{~N}_{2} \mathrm{Na}[\mathrm{M}+\mathrm{Na}]^{+}: 184.9643$, found: 186.9643 .<smiles>Nc1ncc(Cl)nc1Cl</smiles>

\section{(24) 3,5-dichloropyrazin-2-amine}

White solid, $38.2 \mathrm{mg} ;{ }^{1} \mathrm{H}$ NMR $\left(400 \mathrm{MHz}, \mathrm{CDCl}_{3}\right) \delta 7.96(\mathrm{~s}, 1 \mathrm{H}), 5.02$ (brs, 2H) ppm; ${ }^{13} \mathrm{C} \mathrm{NMR}(100 \mathrm{MHz}$, $\left.\mathrm{CDCl}_{3}\right) \delta 150.3,140.0,134.6,131.4$ ppm. HRMS $\left(\mathrm{ESI}^{+}\right) \mathrm{m} / z$ calcd. for $\mathrm{C}_{4} \mathrm{H}_{4} \mathrm{Cl}_{2} \mathrm{~N}_{3}[\mathrm{M}+\mathrm{H}]^{+}: 163.9776$, found: 163.9775 .<smiles>ClNc1ccc2ccccc2n1</smiles>

\section{(25) N-chloroquinolin-2-amine}

Yellow solid, $49.7 \mathrm{mg}$, m.p. 95-97 ${ }^{\circ} \mathrm{C} ;{ }^{1} \mathrm{H}$ NMR $(400 \mathrm{MHz}, \mathrm{CDCl} 3) \delta 7.41(\mathrm{~m}, 2 \mathrm{H}), 7.13(\mathrm{~m}, 2 \mathrm{H}), 6.64(\mathrm{~d}, \mathrm{~J}=$ $2.0 \mathrm{~Hz}, 1 \mathrm{H}) \mathrm{ppm} ;{ }^{13} \mathrm{C} \mathrm{NMR}\left(100 \mathrm{MHz}, \mathrm{CDCl}_{3}\right) \delta 157.1,136.8,133.8,130.3,127.8,122.9,120.6,119.6$, 114.5 ppm. HRMS $\left(\mathrm{ESI}^{+}\right) \mathrm{m} / z$ calcd. for $\mathrm{C}_{9} \mathrm{H}_{7} \mathrm{CIN}_{2} \mathrm{Na}[\mathrm{M}+\mathrm{Na}]^{+}:$201.0189, found: 201.0193.<smiles>Nc1cnc2ccccc2c1Cl</smiles>

\section{(26) 4-chloroquinolin-3-amine}

Black solid, $33.1 \mathrm{mg}$, m.p. $137-139{ }^{\circ} \mathrm{C} ;{ }^{1} \mathrm{H}$ NMR (400 MHz, CDCl $) \delta 8.54(\mathrm{~s}, 1 \mathrm{H}), 8.01(\mathrm{~m}, 2 \mathrm{H}), 7.53(\mathrm{~m}$, 2H), 4.32 (brs, 1H) ppm; ${ }^{13} \mathrm{C}$ NMR $\left(100 \mathrm{MHz}, \mathrm{CDCl}_{3}\right) \delta 142.9,142.0,136.5,129.5,127.9,126.7,126.0$, 122.1, 120.0 ppm. HRMS (ESI) $\mathrm{m} / z$ calcd. for $\mathrm{C}_{9} \mathrm{H}_{7} \mathrm{ClN}_{2} \mathrm{Na}[\mathrm{M}+\mathrm{Na}]^{+}:$201.0189, found: 201.0191 .<smiles>Nc1ncc2ccccc2c1Cl</smiles> 
Yellow solid, $35.3 \mathrm{mg}$, m.p. $62-63{ }^{\circ} \mathrm{C} ;{ }^{1} \mathrm{H}$ NMR $\left(400 \mathrm{MHz}, \mathrm{CDCl}_{3}\right) \delta 8.78(\mathrm{~s}, 1 \mathrm{H}), 7.94(\mathrm{~d}, J=0.8 \mathrm{~Hz}, 1 \mathrm{H})$, $7.92(\mathrm{~d}, J=0.8 \mathrm{~Hz}, 1 \mathrm{H}), 7.64(\mathrm{t}, J=8.0 \mathrm{~Hz}, 1 \mathrm{H}), 7.32(\mathrm{t}, J=8.0 \mathrm{~Hz}, 1 \mathrm{H}), 4.90$ (brs, 2H) ppm; ${ }^{13} \mathrm{C}$ NMR $(100$ $\mathrm{MHz}_{\mathrm{CDCl}}$ ) $\delta 150.6,135.4,131.5,127.9,124.7,123.4,121.2,105.8 \mathrm{ppm}$. HRMS (ESI) $\mathrm{m} / \mathrm{z}$ calcd. for $\mathrm{C}_{9} \mathrm{H}_{8} \mathrm{ClN}_{2}[\mathrm{M}+\mathrm{H}]^{+}:$179.0370, found: 179.0373 .<smiles>CNc1ncc(Cl)cn1</smiles>

\section{(28) 5-chloro-N-methylpyrimidin-2-amine}

White solid, $32.6 \mathrm{mg}$, m.p.69-70 ${ }^{\circ} \mathrm{C} ;{ }^{1} \mathrm{H}$ NMR (400 MHz, CDCl$) \delta 8.21(\mathrm{~s}, 2 \mathrm{H}), 5.19$ (brs, $1 \mathrm{H}$ ), 2.97 (s, 3H) ppm; $\left.{ }^{13} \mathrm{C} \mathrm{NMR} \mathrm{(100} \mathrm{MHz,} \mathrm{CDCl}_{3}\right) \delta 161.1,156.1,118.7,28.6$ ppm. HRMS (ESI) $\mathrm{m} / z$ calcd. for $\mathrm{C}_{5} \mathrm{H}_{7} \mathrm{CIN}_{3}$ $[\mathrm{M}+\mathrm{H}]^{+}:$144.0322, found: 144.0327 .<smiles>Nc1ncc(Cl)c(-c2cccnc2)n1</smiles>

\section{(29) 5-chloro-4-(pyridin-3-yl)pyrimidin-2-amine}

White solid, $58.1 \mathrm{mg} ;{ }^{1} \mathrm{H}$ NMR $\left(400 \mathrm{MHz}, \mathrm{DMSO}-\mathrm{d}_{6}\right) \delta 8.86(\mathrm{~d}, J=7.0 \mathrm{~Hz}, 1 \mathrm{H}), 8.67(\mathrm{dd}, J=4.8,1.6 \mathrm{~Hz}$, $1 \mathrm{H}), 8.40(\mathrm{~s}, 1 \mathrm{H}), 8.10(\mathrm{dt}, J=8.0,1.6 \mathrm{~Hz}, 1 \mathrm{H}), 7.53(\mathrm{~m}, 1 \mathrm{H}), 7.07(\mathrm{~s}, 1 \mathrm{H}) \mathrm{ppm} ;{ }^{13} \mathrm{C} \mathrm{NMR}\left(100 \mathrm{MHz}, \mathrm{CDCl}_{3}\right)$ $\delta$ 162.6, 160.3, 158.9, 150.9, 149.7, 136.8, 132.5, 123.6, 115.5 ppm. HRMS (ESI) $\mathrm{m} / z$ calcd. for $\mathrm{C}_{9} \mathrm{H}_{8} \mathrm{CIN}_{4}$ $[\mathrm{M}+\mathrm{H}]^{+}:$207.0431, found: 207.0429 .

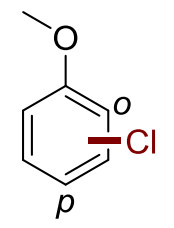

(30 p) 1-chloro-4-methoxybenzene. (30 o) 1-chloro-2-methoxybenzene

The spectroscopic date for these compounds were identical to those reported in the literature: Gary A. Molander and Livia N. Cavacanti J. Org. Chem. 2011, 76, 7195-7203.; Hong Wu and John Hynes, Jr. Org. Lett, 2010, Vol. 12, No. 6, 1192-1195. 
<smiles>COc1ccc(NC(C)=O)c(Cl)c1</smiles>

(31) N-(2-chloro-4-methoxyphenyl)acetamide

Known compound. ${ }^{5}$ White solid, 30.5 mg, m.p. $101-103{ }^{\circ} \mathrm{C} ;{ }^{1} \mathrm{H}$ NMR $\left(400 \mathrm{MHz}, \mathrm{CDCl}_{3}\right) \delta 8.14(\mathrm{~d}, \mathrm{~J}=8.9$ $\mathrm{Hz}, 1 \mathrm{H}), 7.39$ (brs, 1H), $6.91(\mathrm{~s}, 1 \mathrm{H}), 6.81(\mathrm{~d}, \mathrm{~J}=8.4 \mathrm{~Hz}, 1 \mathrm{H}), 3.77(\mathrm{~s}, 3 \mathrm{H}), 2.20(\mathrm{~s}, 3 \mathrm{H}) \mathrm{ppm} ;{ }^{13} \mathrm{C}$ NMR $(100$ $\left.\mathrm{MHz}_{2} \mathrm{CDCl}_{3}\right) \delta 169.5,157.7,129.3,125.5,124.8,115.9,114.6,57.0,25.9$ ppm. HRMS (ESI) $\mathrm{m} / \mathrm{z}$ calcd. for $\mathrm{C}_{9} \mathrm{H}_{11} \mathrm{ClNO}_{2}[\mathrm{M}+\mathrm{H}]^{+}:$200.0472, found: 200.0473 .<smiles>CS(=O)(=O)c1cc(Cl)c(N)c(Cl)c1</smiles>

\section{(32) 2,6-dichloro-4-(methylsulfonyl)aniline}

White solid, $62.4 \mathrm{mg}$, m.p. $147-149{ }^{\circ} \mathrm{C} ;{ }^{1} \mathrm{H}$ NMR (400 MHz, CDCl $) \delta 7.75$ (s, 2H), 5.03 (brs, $2 \mathrm{H}$ ), 3.03 (s, $2 \mathrm{H}) ;{ }^{13} \mathrm{C}$ NMR $\left(100 \mathrm{MHz}, \mathrm{CDCl}_{3}\right) \delta 146.1,130.4,128.7,120.3,46.4 \mathrm{ppm}$. HRMS (ESI) $\mathrm{m} / z$ calcd. for $\mathrm{C}_{7} \mathrm{H}_{7} \mathrm{Cl}_{2} \mathrm{NNaO}_{2} \mathrm{~S}[\mathrm{M}+\mathrm{Na}]^{+}:$261.9467, found: 261.9471 .<smiles>Nc1c(Cl)cc(Cl)cc1Cl</smiles>

\section{(33) 2,4,6-trichloroaniline}

Known compound. ${ }^{6}$ Yellow solid, $44.5 \mathrm{mg}$, m.p. $62-63{ }^{\circ} \mathrm{C} ;{ }^{1} \mathrm{H}$ NMR $\left(400 \mathrm{MHz}, \mathrm{CDCl}_{3}\right) \delta 7.19(\mathrm{~s}, 2 \mathrm{H}), 4.43$ (brs, $2 \mathrm{H}) ;{ }^{13} \mathrm{C} \mathrm{NMR}\left(100 \mathrm{MHz}, \mathrm{CDCl}_{3}\right) \delta 140.4,129.0,123.2,121.1 \mathrm{ppm}$.<smiles>Nc1c(Cl)cc(Cl)c(Cl)c1Cl</smiles>

(34) 2,3,4,6-tetrachloroaniline

Yellow solid, $62.5 \mathrm{mg}$, m.p. $78-79{ }^{\circ} \mathrm{C} ;{ }^{1} \mathrm{H}$ NMR $\left(400 \mathrm{MHz}, \mathrm{CDCl}_{3}\right) \delta 7.32(\mathrm{~s}, 1 \mathrm{H}), 4.58$ (brs, $\left.2 \mathrm{H}\right) ;{ }^{13} \mathrm{C}$ NMR $\left(100 \mathrm{MHz}, \mathrm{CDCl}_{3}\right) \delta$ 141.6, 131.7, 129.4, 122.5, 120.1, 118.8 ppm. HRMS (ESI) $\mathrm{m} / z$ calcd. for $\mathrm{C}_{6} \mathrm{H}_{4} \mathrm{Cl}_{4} \mathrm{~N}$ $[\mathrm{M}+\mathrm{H}]^{+}:$229.9092, found: 229.9091. 
<smiles>Nc1ccc(C(F)(F)F)cc1Cl</smiles>

(35) 1-chloro-4-(trifluoromethyl)aniline

Known compound. ${ }^{7}$ Yellow oil, $45.6 \mathrm{mg} ;{ }^{1} \mathrm{H}$ NMR $\left(400 \mathrm{MHz}, \mathrm{CDCl}_{3}\right) \delta 7.51(\mathrm{~s}, 1 \mathrm{H}), 7.30(\mathrm{~d}, J=8.4 \mathrm{~Hz}, 1 \mathrm{H})$, $6.78(\mathrm{~d}, J=8.4 \mathrm{~Hz}, 1 \mathrm{H}), 4.26$ (brs, $1 \mathrm{H}) ;{ }^{13} \mathrm{C} \mathrm{NMR}\left(100 \mathrm{MHz}, \mathrm{CDCl}_{3}\right) \delta 145.8126 .7$ (q, J = 3.9 Hz), 124.8 (q, $J=3.7 \mathrm{~Hz}), 120.7(q, J=33.3 \mathrm{~Hz}), 118.4,114.9 \mathrm{ppm}$.<smiles>CCOC(=O)C(Cl)(Cl)C(=O)c1ccccc1</smiles>

\section{(36) ethyl 2,2-dichloro-3-oxo-3-phenylpropanoate}

Yellow oil, $64.0 \mathrm{mg} ;{ }^{1} \mathrm{H}$ NMR $\left(400 \mathrm{MHz}, \mathrm{CDCl}_{3}\right) \delta 8.03(\mathrm{~d}, \mathrm{~J}=7.7 \mathrm{~Hz}, 2 \mathrm{H}), 7.60(\mathrm{t}, \mathrm{J}=7.3 \mathrm{~Hz}, 1 \mathrm{H}), 7.47(\mathrm{t}, \mathrm{J}$ $=7.7 \mathrm{~Hz}, 2 \mathrm{H}), 4.31(\mathrm{q}, \mathrm{J}=7.0 \mathrm{~Hz}, 2 \mathrm{H}), 1.17(\mathrm{t}, \mathrm{J}=6.9 \mathrm{~Hz}, 3 \mathrm{H}) \mathrm{ppm} ;{ }^{13} \mathrm{C} \mathrm{NMR}\left(100 \mathrm{MHz}, \mathrm{CDCl}_{3}\right) \delta 184.6$, 165.4, 135.6, 132.3, 131.5, 130.0, 83.3, 66.0, 14.9 ppm. HRMS (ESI) m/z calcd. for $\mathrm{C}_{11} \mathrm{H}_{10} \mathrm{Cl}_{2} \mathrm{NaO}_{3}$ $[\mathrm{M}+\mathrm{Na}]^{+}: 282.9899$, found: 282.9907.<smiles></smiles>

(37) 2,8-dichloro-10-ethyl-5,5-difluoro-1,3,7,9-tetramethyl-5H-dipyrrolo[1,2-c:2',1'f][1,3,2]diazaborinin-4-ium-5-uide

Red solid, $90.7 \mathrm{mg} ;{ }^{1} \mathrm{H} \mathrm{NMR}\left(400 \mathrm{MHz}, \mathrm{CDCl}_{3}\right) \delta 2.96(\mathrm{t}, \mathrm{J}=8.0 \mathrm{~Hz}, 2 \mathrm{H}), 2.54(\mathrm{~s}, 6 \mathrm{H}), 2.41(\mathrm{~s}, 6 \mathrm{H}), 1.58(\mathrm{~m}$, 2H), $1.46(\mathrm{~m}, 2 \mathrm{H}), 1.33(\mathrm{~m}, 6 \mathrm{H}), 0.89(\mathrm{t}, J=6.8 \mathrm{~Hz}, 3 \mathrm{H}) \mathrm{ppm} ;{ }^{13} \mathrm{C} \mathrm{NMR}\left(100 \mathrm{MHz}, \mathrm{CDCl}_{3}\right) \delta$ 150.8, 147.5, 135.1, 129.1, 122.5, 31.8, 31.7, 30.2, 29.0, 28.8, 22.5, 14.0, 13.6, 12.3 ppm. HRMS (ESI) $\mathrm{m} / z$ calcd. for $\mathrm{C}_{20} \mathrm{H}_{27} \mathrm{BCl}_{2} \mathrm{NaF}_{2} \mathrm{~N}_{2}[\mathrm{M}+\mathrm{Na}]^{+}:$437.1504, found: 437.1509. 
<smiles></smiles>

(38) 2,8-dichloro-5,5-difluoro-1,3,7,9-tetramethyl-10-phenyl-5H-dipyrrolo[1,2-c:2',1'-

\section{f][1,3,2]diazaborinin-4-ium-5-uide}

Red solid, $95.3 \mathrm{mg}$, m.p. $158-160{ }^{\circ} \mathrm{C} ;{ }^{1} \mathrm{H}$ NMR (400 MHz, $\left.\mathrm{CDCl}_{3}\right) \delta 7.52(\mathrm{t}, J=2.8 \mathrm{~Hz}, 3 \mathrm{H}), 7.25(\mathrm{t}, \mathrm{J}=3.6$ $\mathrm{Hz}, 2 \mathrm{H}), 2.58$ (s, 6H), 1.35 (s, 6H) ppm; $\left.{ }^{13} \mathrm{C} \mathrm{NMR} \mathrm{(100} \mathrm{MHz,} \mathrm{CDCl}\right) \delta 152.5,142.4,138.0,134.2,129.6$, 129.5, 129.4, 127.8, 122.6, 12.4, $11.8 \mathrm{ppm}$. HRMS (ESI) $\mathrm{m} / \mathrm{z}$ calcd. for $\mathrm{C}_{19} \mathrm{H}_{17} \mathrm{BCl}_{2} \mathrm{NaF}_{2} \mathrm{~N}_{2}[\mathrm{M}+\mathrm{Na}]^{+}$: 415.0721, found: 415.0729 .

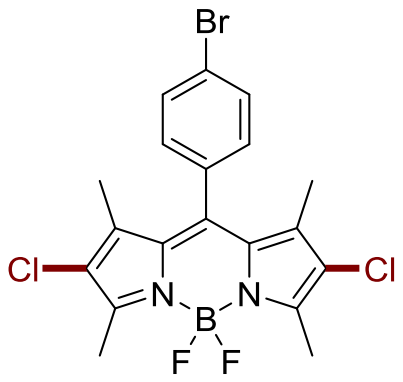

(39) 10-(4-bromophenyl)-2,8-dichloro-5,5-difluoro-1,3,7,9-tetramethyl-5H-dipyrrolo[1,2-c:2',1'f][1,3,2]diazaborinin-4-ium-5-uide

Red solid, $101.5 \mathrm{mg} ;{ }^{1} \mathrm{H}$ NMR $\left(400 \mathrm{MHz}, \mathrm{CDCl}_{3}\right) \delta 7.68(\mathrm{~d}, J=8.4 \mathrm{~Hz}, 2 \mathrm{H}), 7.15(\mathrm{~d}, J=8.4 \mathrm{~Hz}, 2 \mathrm{H}), 2.58$ (s, 2H), 1.40 (s, 2H) ppm; ${ }^{13} \mathrm{C}$ NMR (100 MHz, CDCl 3 ) $\delta$ 153.0, 140.6, 137.7, 133.1, 132.7, 129.6, 129.4, 123.9, 122.9, 12.4, 12.2 ppm. HRMS (ESI) $\mathrm{m} / z$ calcd. for $\mathrm{C}_{19} \mathrm{H}_{16} \mathrm{BBrCl}_{2} \mathrm{NaF}_{2} \mathrm{~N}_{2}[\mathrm{M}+\mathrm{Na}]^{+}: 492.9826$, found: 492.9830 .<smiles></smiles>

(40) 2,8-dichloro-5,5-difluoro-10-(4-methoxyphenyl)-1,3,7,9-tetramethyl-5H-dipyrrolo[1,2-c:2',1'- 
Red solid, 79.8 mg, m.p. $195-196{ }^{\circ} \mathrm{C} ;{ }^{1} \mathrm{H}$ NMR (400 MHz, $\left.\mathrm{CDCl}_{3}\right) \delta 7.14$ (d, J=8.8 Hz, 2H), 7.03 (d, J=8.8 $\mathrm{Hz}, 2 \mathrm{H}), 3.89(\mathrm{~s}, 3 \mathrm{H}), 2.57(\mathrm{~s}, 6 \mathrm{H}), 1.41(\mathrm{~s}, 6 \mathrm{H}) \mathrm{ppm} ;{ }^{13} \mathrm{C} \mathrm{NMR}\left(100 \mathrm{MHz}, \mathrm{CDCl}_{3}\right) \delta$ 153.9, 152.3, 142.6, 138.0, 130.0, 129.1, 126.1, 122.5, 114.8, 55.38, 12.4, $12.1 \mathrm{ppm}$. HRMS (ESI) $\mathrm{m} / \mathrm{z}$ calcd. for $\mathrm{C}_{20} \mathrm{H}_{19} \mathrm{BCl}_{2} \mathrm{NaF}_{2} \mathrm{~N}_{2} \mathrm{O}[\mathrm{M}+\mathrm{Na}]^{+}:$445.0827, found: 445.0836 .

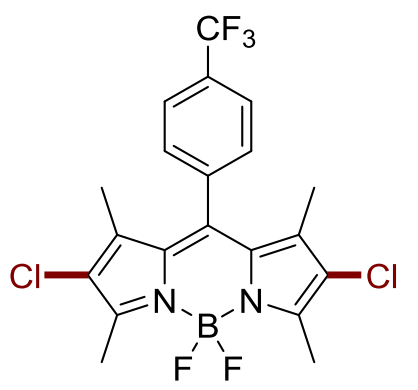

(41) 2,8-dichloro-5,5-difluoro-1,3,7,9-tetramethyl-10-(4-(trifluoromethyl)phenyl)-5H-dipyrrolo[1,2$\left.c: 2^{\prime}, 1^{\prime}-f\right][1,3,2]$ diazaborinin-4-ium-5-uide

Red solid, $107.6 \mathrm{mg} ;{ }^{1} \mathrm{H}$ NMR $\left(400 \mathrm{MHz}, \mathrm{CDCl}_{3}\right) \delta 7.81$ (d, J=8 Hz, 2H), $7.44(\mathrm{~d}, J=8 \mathrm{~Hz}, 2 \mathrm{H}), 2.59(\mathrm{~s}, 6 \mathrm{H})$, $1.33(\mathrm{~s}, 6 \mathrm{H}) \mathrm{ppm} ;{ }^{13} \mathrm{C} \mathrm{NMR}\left(100 \mathrm{MHz}, \mathrm{CDCl}_{3}\right) \delta 153.3,140.1,138.0,137.6,132.1,131.8,129.2,128.6$, 126.3, 123.1, 12.4, 12.0 ppm. HRMS (ESI) $\mathrm{m} / z$ calcd. for $\mathrm{C}_{20} \mathrm{H}_{16} \mathrm{BCl}_{2} \mathrm{NaF}_{5} \mathrm{~N}_{2}[\mathrm{M}+\mathrm{Na}]^{+}:$483.0595, found: 483.0601.

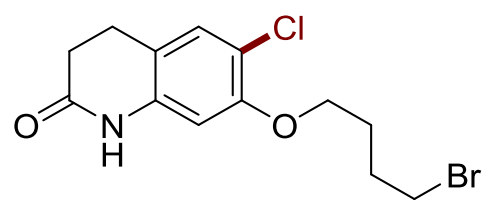

(43) 7-(4-bromobutoxy)-6-chloro-3,4-dihydroquinolin-2(1H)-one

White solid, $82.4 \mathrm{mg}$, m.p. $101-103{ }^{\circ} \mathrm{C} ;{ }^{1} \mathrm{H}$ NMR $\left(400 \mathrm{MHz}, \mathrm{CDCl}_{3}\right) \delta 8.99(\mathrm{~s}, 1 \mathrm{H}), 7.11(\mathrm{~s}, 1 \mathrm{H}), 6.40(\mathrm{~s}$, $1 \mathrm{H}), 4.01(\mathrm{t}, \mathrm{J}=5.2 \mathrm{~Hz}, 2 \mathrm{H}), 3.50(\mathrm{t}, \mathrm{J}=5.8 \mathrm{~Hz}, 2 \mathrm{H}), 2.86(\mathrm{t}, \mathrm{J}=6.8 \mathrm{~Hz}, 2 \mathrm{H}), 2.58(\mathrm{t}, \mathrm{J}=7.4 \mathrm{~Hz}, 2 \mathrm{H}), 2.09$ (m, 2H), 1.97 (m,2H) ppm; ${ }^{13} \mathrm{CNMR}\left(100 \mathrm{MHz} \mathrm{CDCl}_{3}\right) \delta 173.1,155.0,138.2,130.5,117.8,102.9,69.8$, 34.8, 32.1, 30.8, 29.0, 25.8 ppm. HRMS (ESI) $\mathrm{m} / z$ calcd. for $\mathrm{C}_{13} \mathrm{H}_{15} \mathrm{ClBrNaNO}{ }_{2}[\mathrm{M}+\mathrm{Na}]^{+}: 353.9867$, found: 353.9874 .<smiles>O=C1CCc2cc(Cl)c(OCCCCN3CCN(c4cccc(Cl)c4Cl)CC3)cc2N1</smiles>

(45) 6-chloro-7-(4-(4-(2,3-dichlorophenyl)piperazin-1-yl)butoxy)-3,4-dihydroquinolin-2(1H)-one 
White solid, $131.3 \mathrm{mg}$, m.p. $82-84{ }^{\circ} \mathrm{C} ;{ }^{1} \mathrm{H}$ NMR (400 MHz, $\mathrm{CDCl}_{3}$ ) $\delta 8.99(\mathrm{~s}, 1 \mathrm{H}), 7.13(\mathrm{~m}, 3 \mathrm{H}), 6.94$ (dd, J = 6.7, $2.7 \mathrm{~Hz}, 1 \mathrm{H}), 6.41(\mathrm{~s}, 1 \mathrm{H}), 4.02(\mathrm{t}, \mathrm{J}=5.9 \mathrm{~Hz}, 2 \mathrm{H}), 3.09$ (brs, $4 \mathrm{H}), 2.94(\mathrm{~s}, 3 \mathrm{H}), 2.87(\mathrm{t}, \mathrm{J}=3.4 \mathrm{~Hz}, 2 \mathrm{H})$, $2.84(\mathrm{~s}, 1 \mathrm{H}), 2.71(\mathrm{brs}, 4 \mathrm{H}), 2.57(\mathrm{~m}, 4 \mathrm{H}), 1.87(\mathrm{~m}, 2 \mathrm{H}), 1.79(\mathrm{~m} .2 \mathrm{H}) \mathrm{ppm} ;{ }^{13} \mathrm{C} \mathrm{NMR}\left(100 \mathrm{MHz}, \mathrm{CDCl}_{3}\right) \delta$ 173.2, 155.2, 152.5, 138.1, 135.4, 130.5, 128.9, 128.8, 126.0, 120.0, 117.9, 117.6, 102.8, 70.6, 59.4, 54.6, 52.4, 37.9, 32.1, 28.4, 25.8, 24.5 ppm. HRMS (ESI) $m / z$ calcd. for $\mathrm{C}_{23} \mathrm{H}_{27} \mathrm{Cl}_{3} \mathrm{~N}_{3} \mathrm{O}_{2}[\mathrm{M}+\mathrm{H}]^{+}$: 482.1163, found: 482.1164 .

\section{References}

1. Matoušek, V.; Pietrasiak, E.; Schwenk, R.; Togni, A. J. Org. Chem. 2013, 78, 6763.

2. Nepomnyashchii, A. B.; Bröring, M.; Ahrens, J.; Bard, A. J. J. Am. Chem. Soc. 2011. 133. 8633.

3. Rodriguez, R. A.; Pan, C. M.; Yabe, Y.; Kawamata, Y.; Eastgate, M. D.; Baran, P. S. J. Am. Chem. Soc. 2014, 136, 6908.

4. Guney, T.; Lee, J. J.; Kraus, G. A. Org. Lett. 2014, 16, 1124.

5. Wan, X.; Ma, Z.; Li, B.; Zhang, K.; Cao, S.; Zhang, S.; Shi, Z. J. Am. Chem. Soc. 2006, 128, 7416.

6. Jagadeesh, R. V.; Natte, K.; Junge, H.; Beller, M. ACS Catal. 2015, 5, 1526.

7. Kino, T.; Magase, Y.; Ohtsuka, Y.; Yamamoto, K.; Uraguchi, D.; Tokuhisa, K.; Yamakawa, T. J. Fluorine Chem. 2010, 131, 98. 
10. ${ }^{1} \mathrm{H}$ and ${ }^{13} \mathrm{C}$ NMR spectral

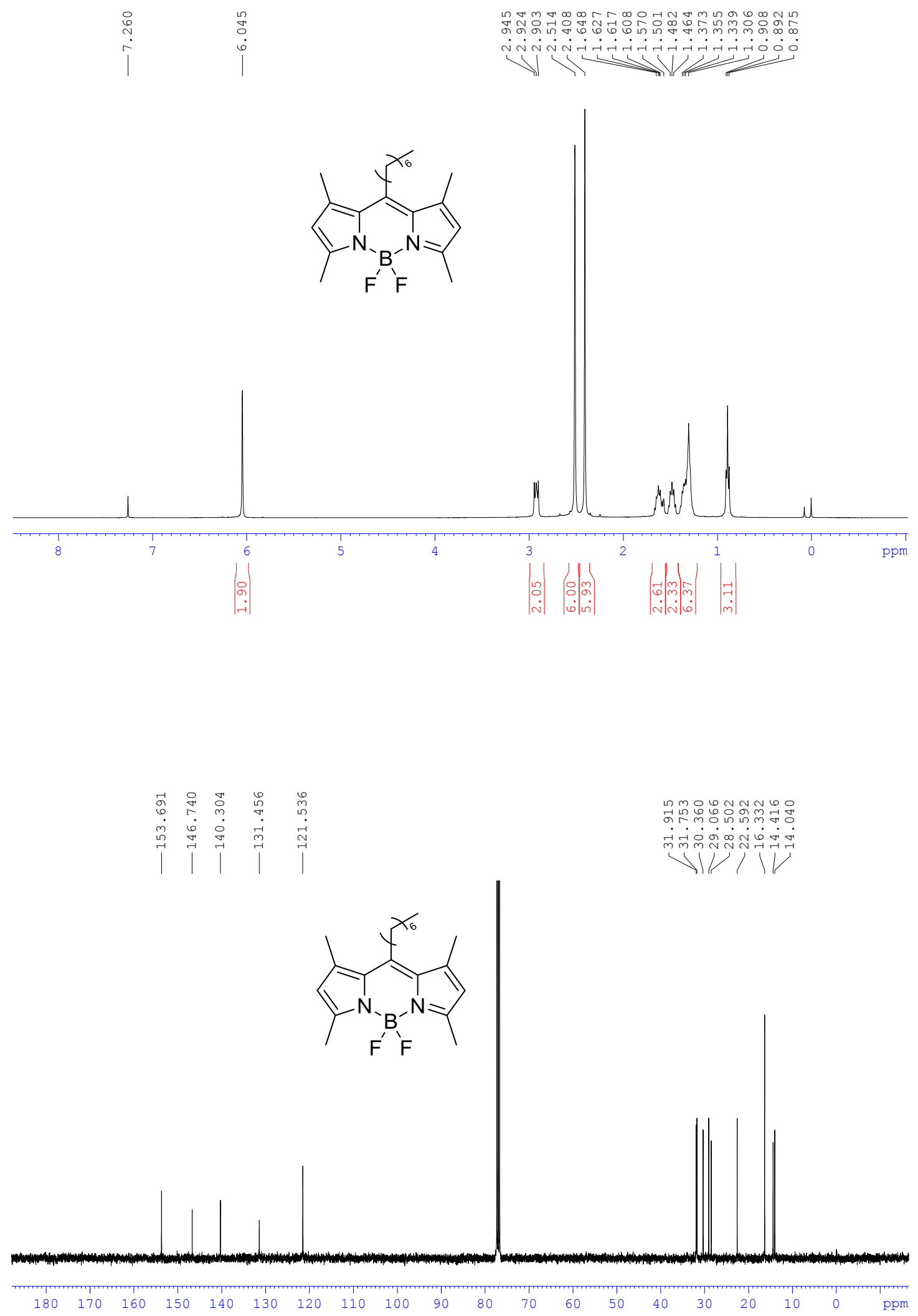



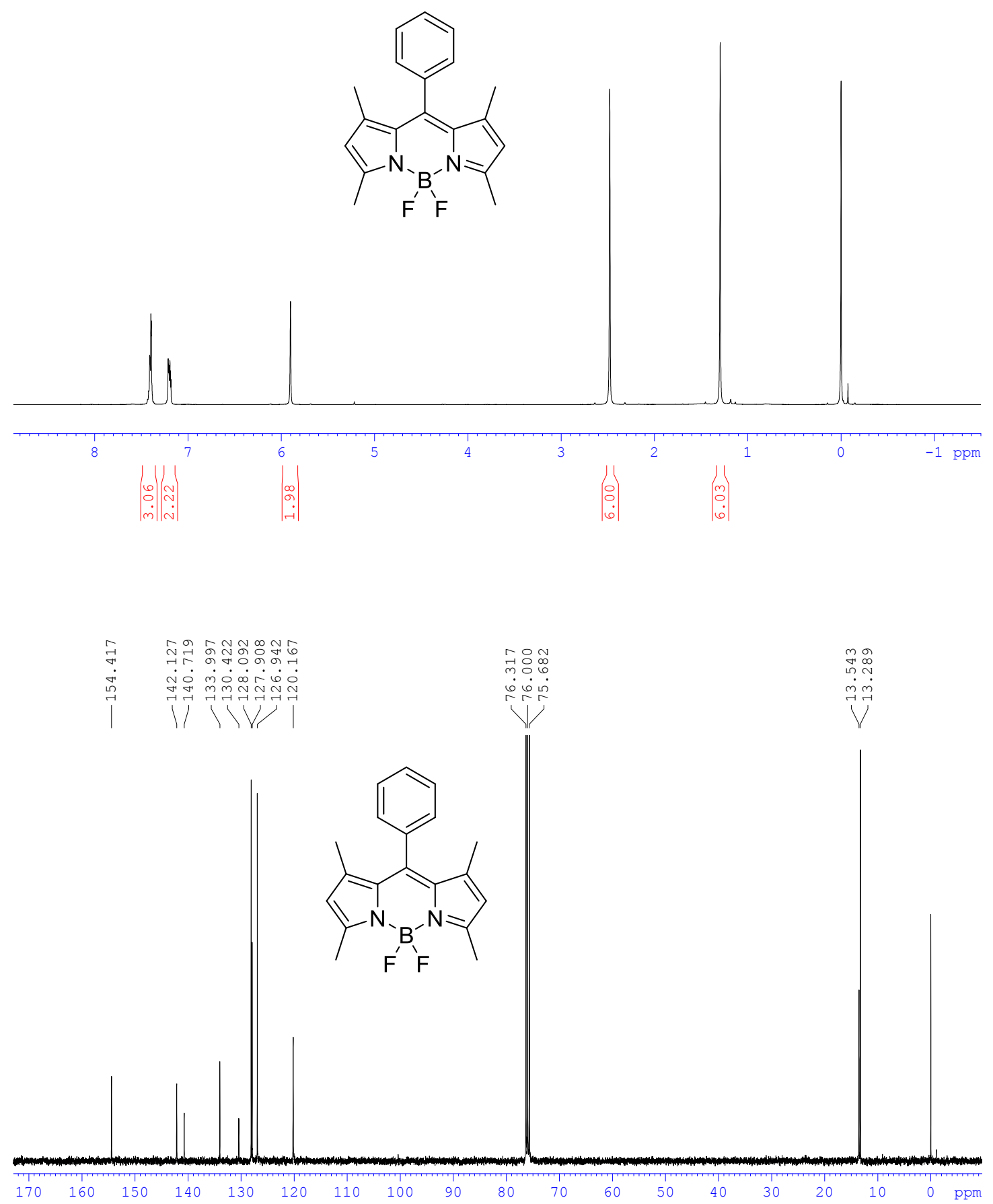


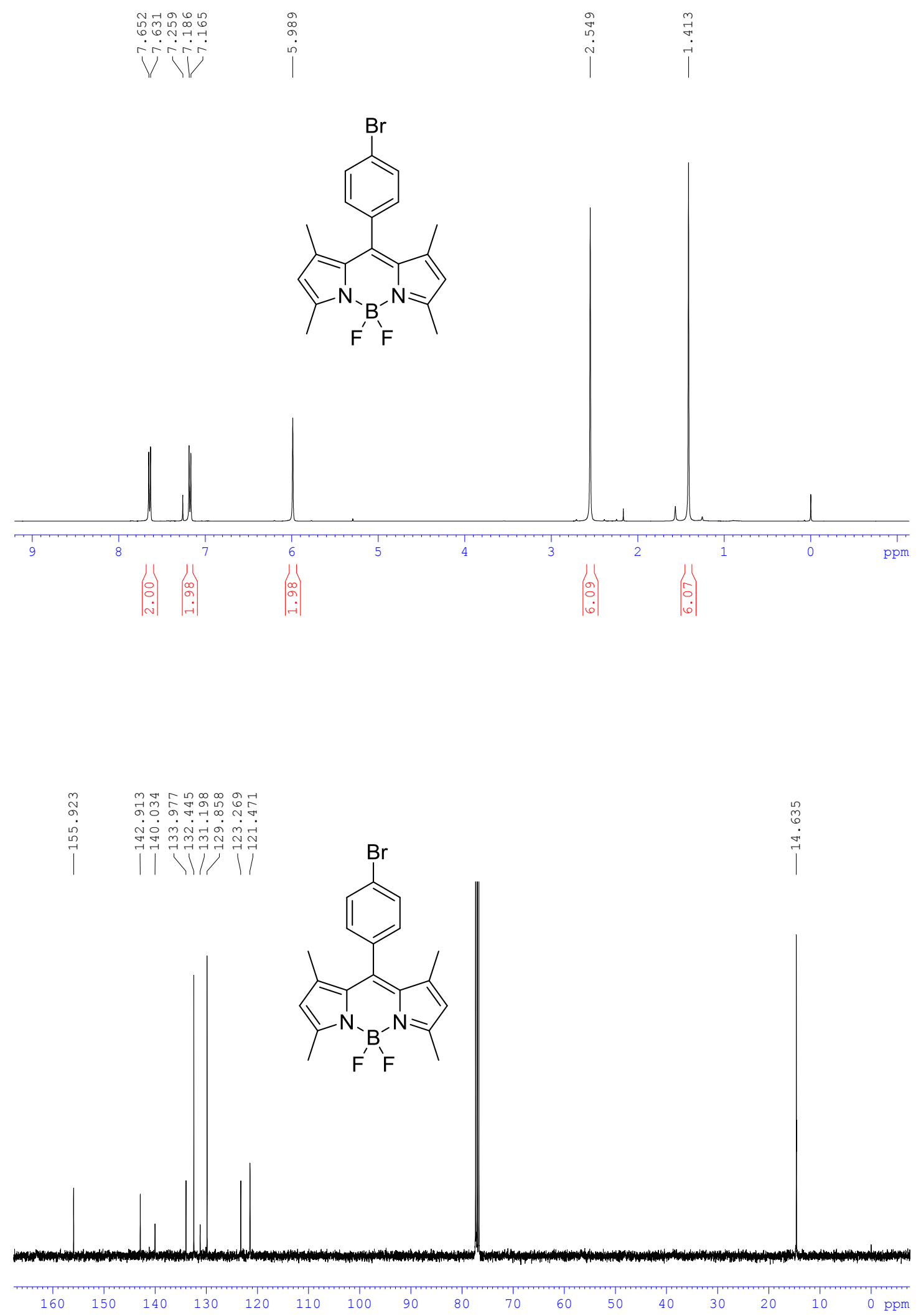




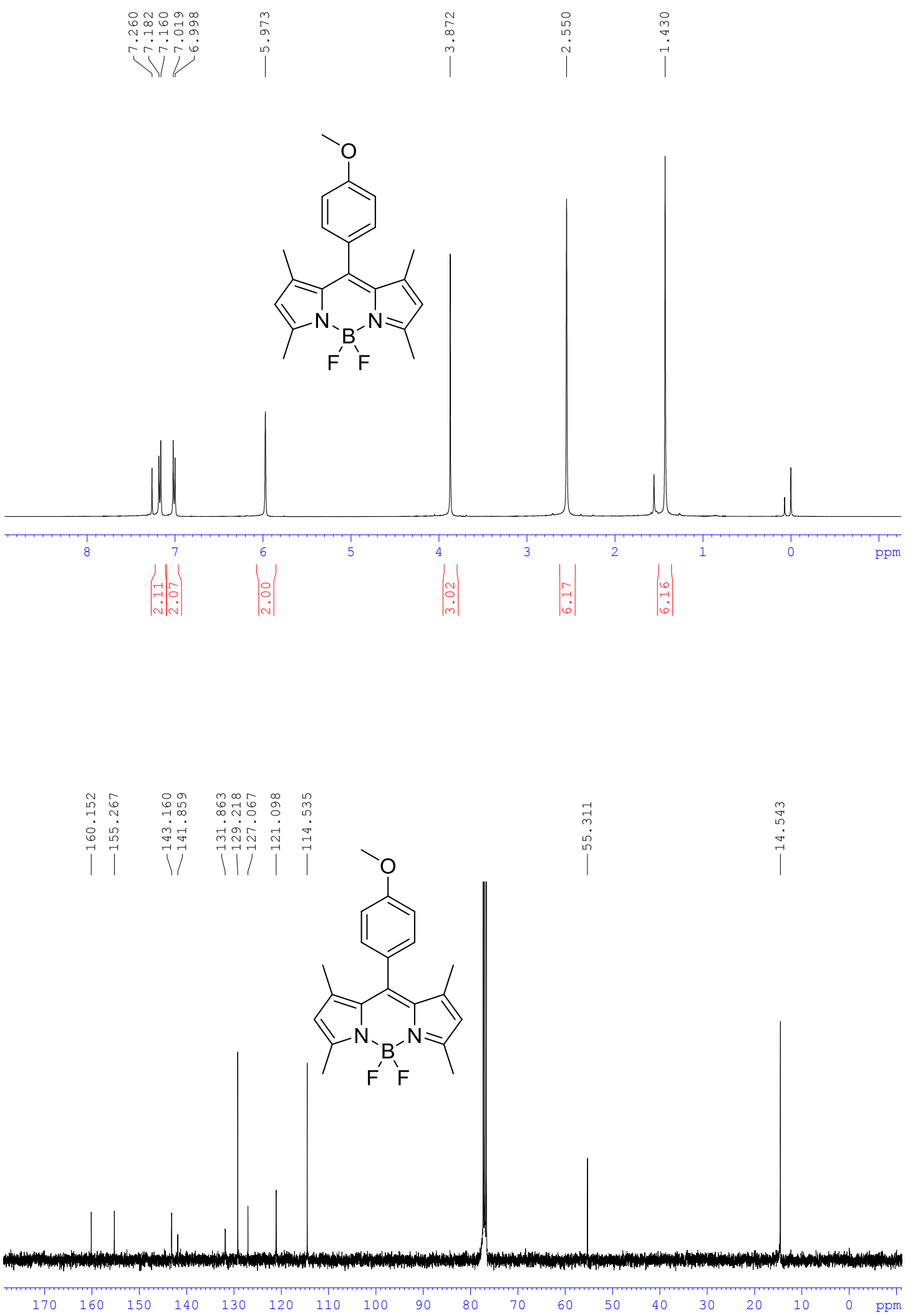




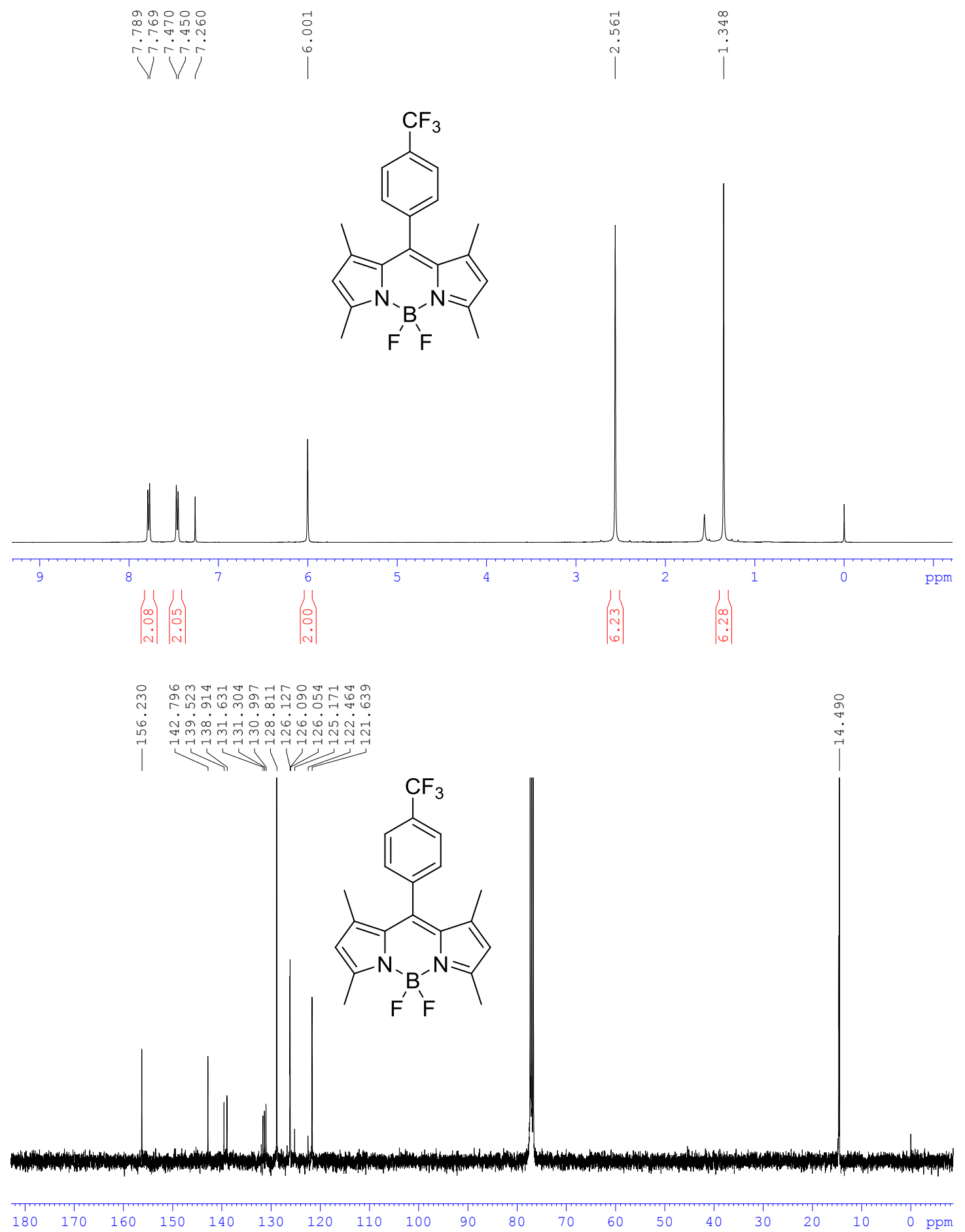



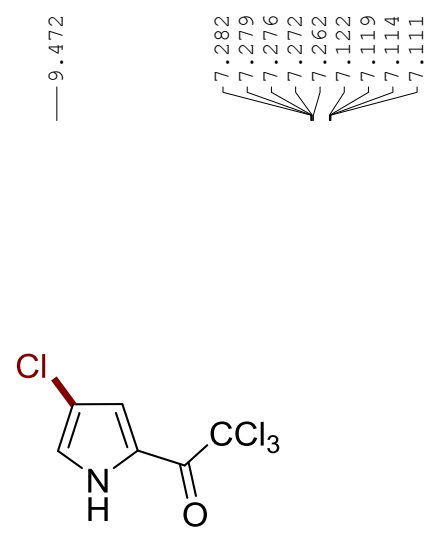

(2)
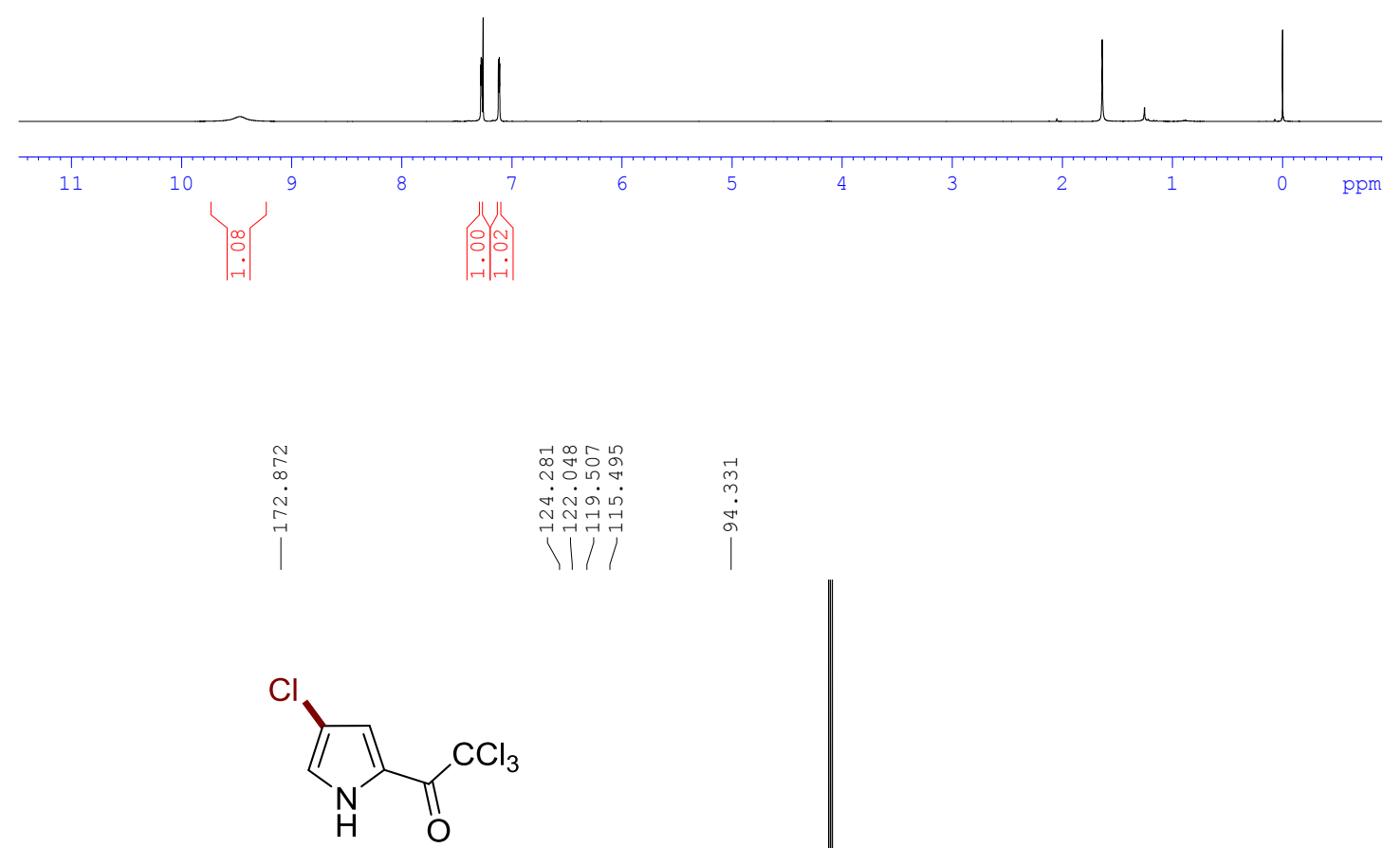

(2)

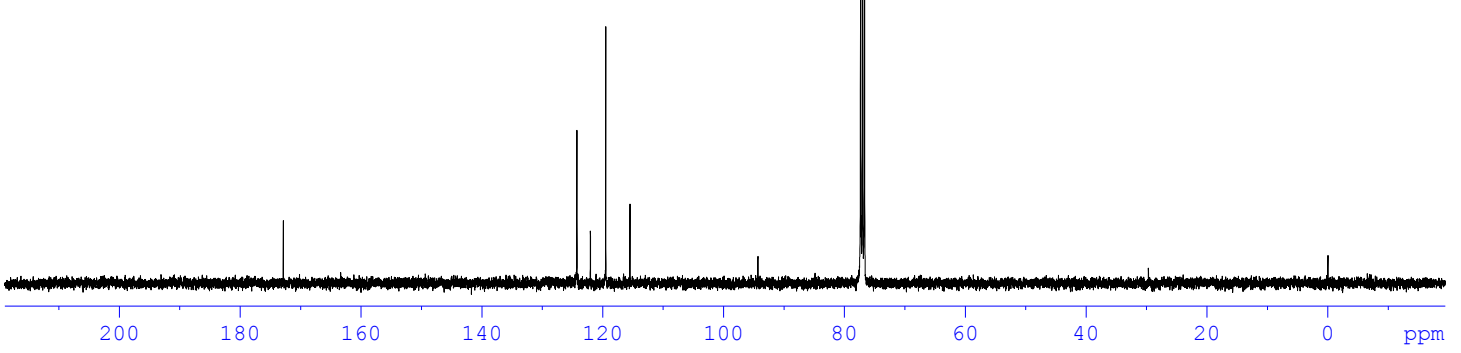



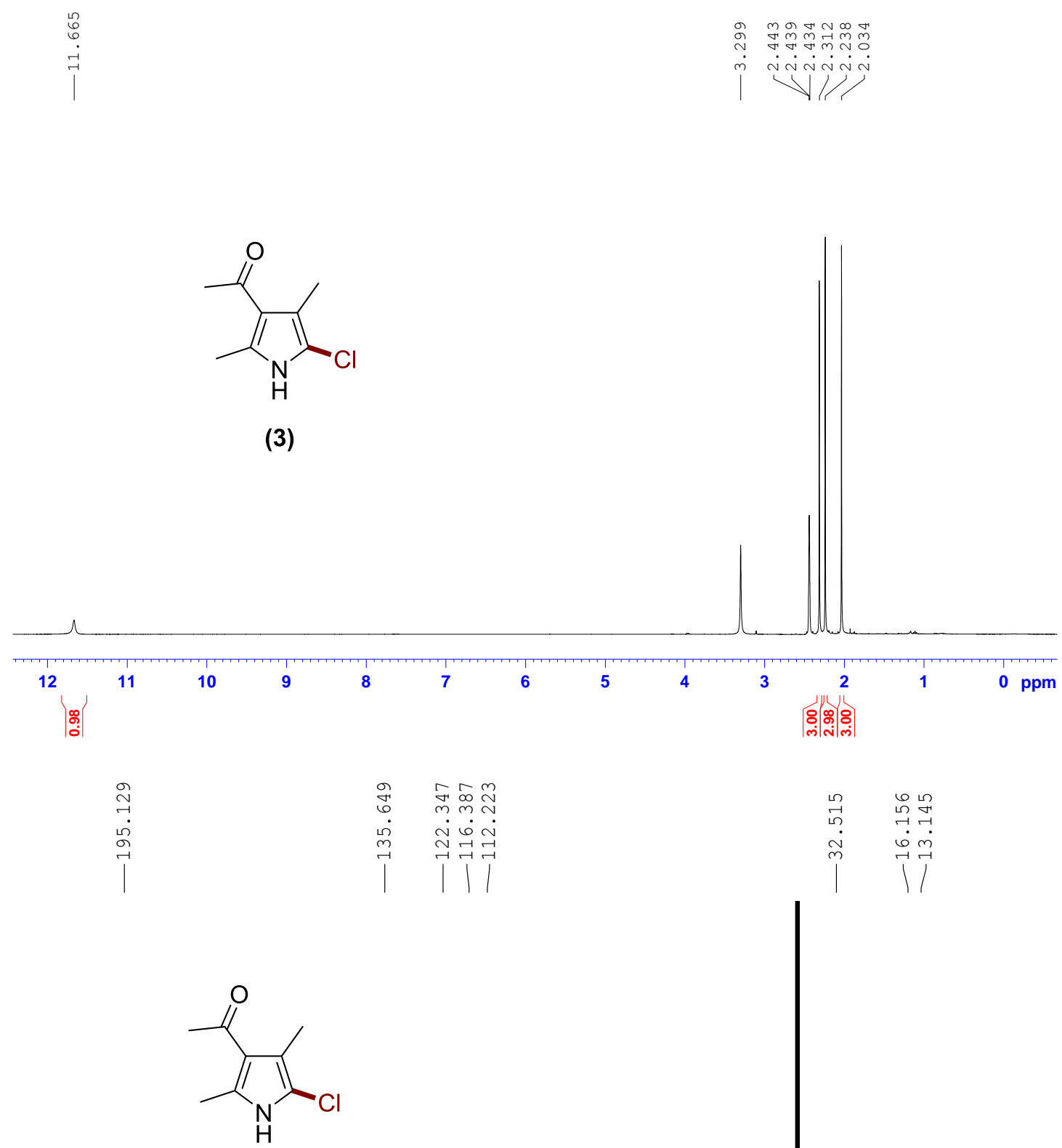

(3)

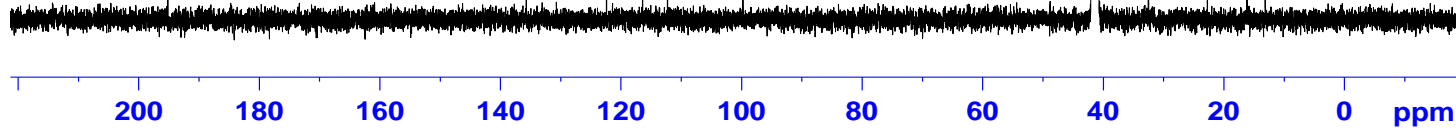




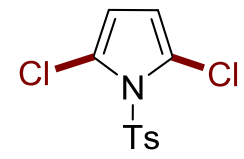

(4)

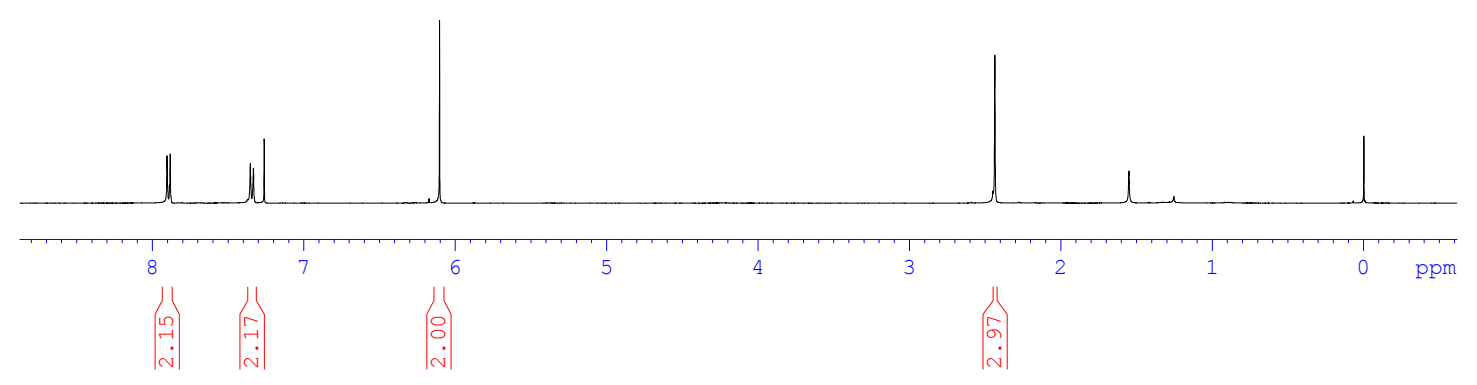

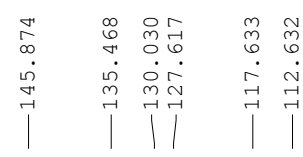

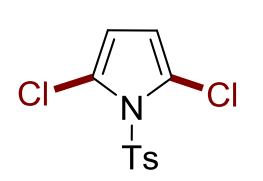

(4)

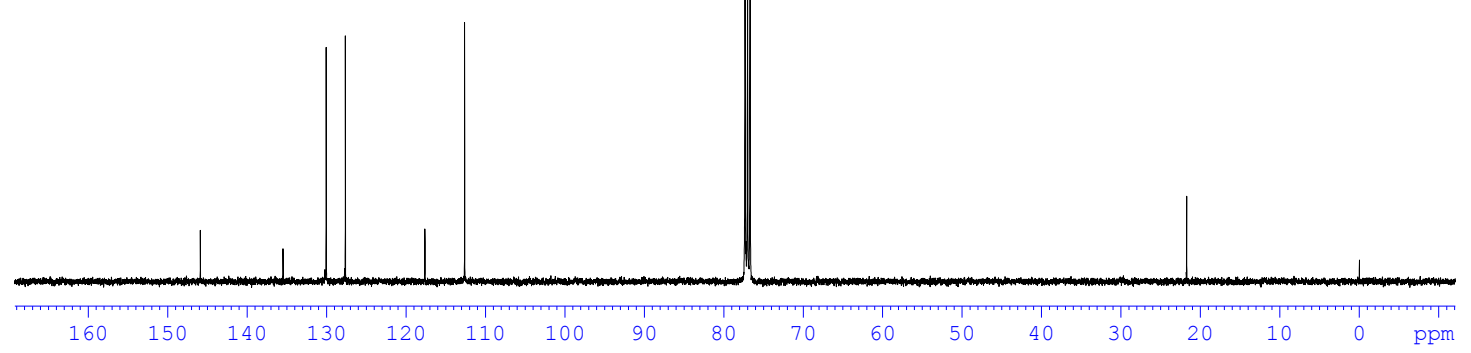




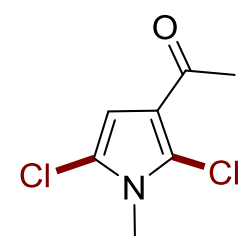

(5)
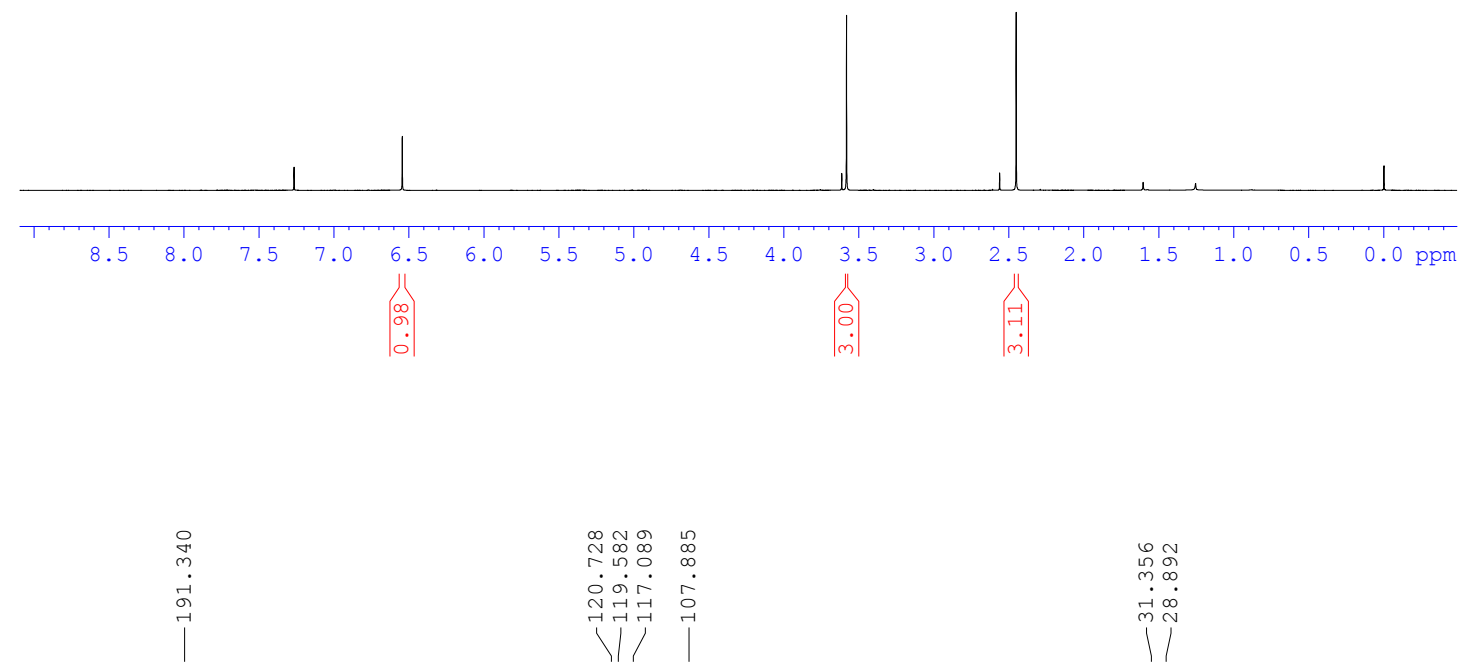

(5)

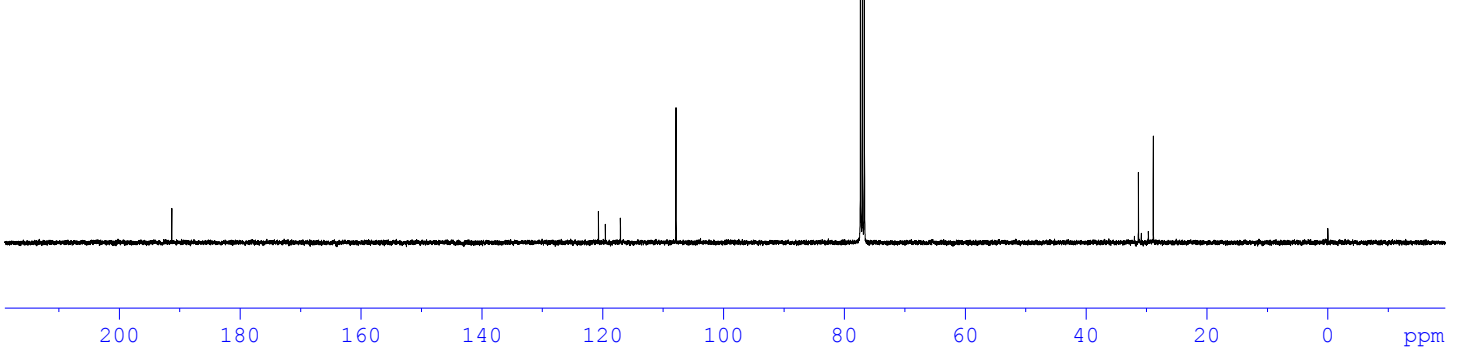




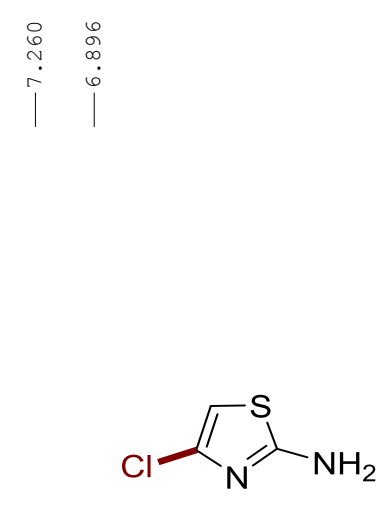

(6)
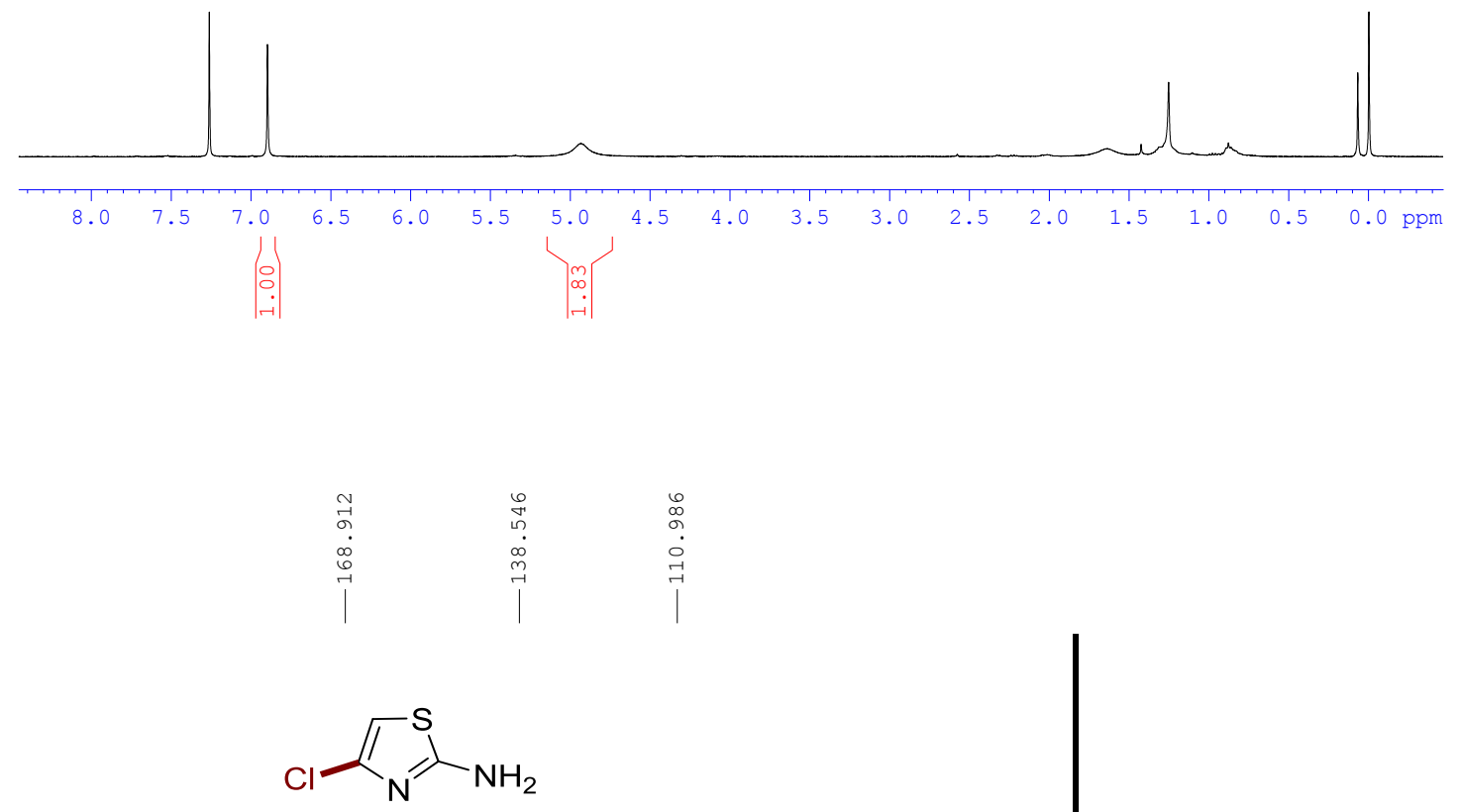

(6)

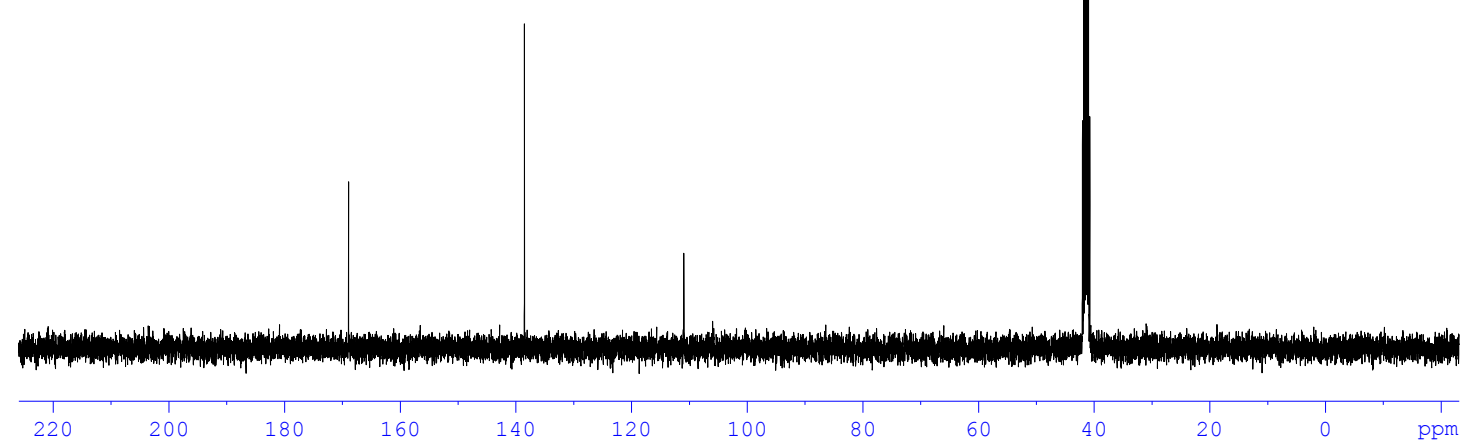




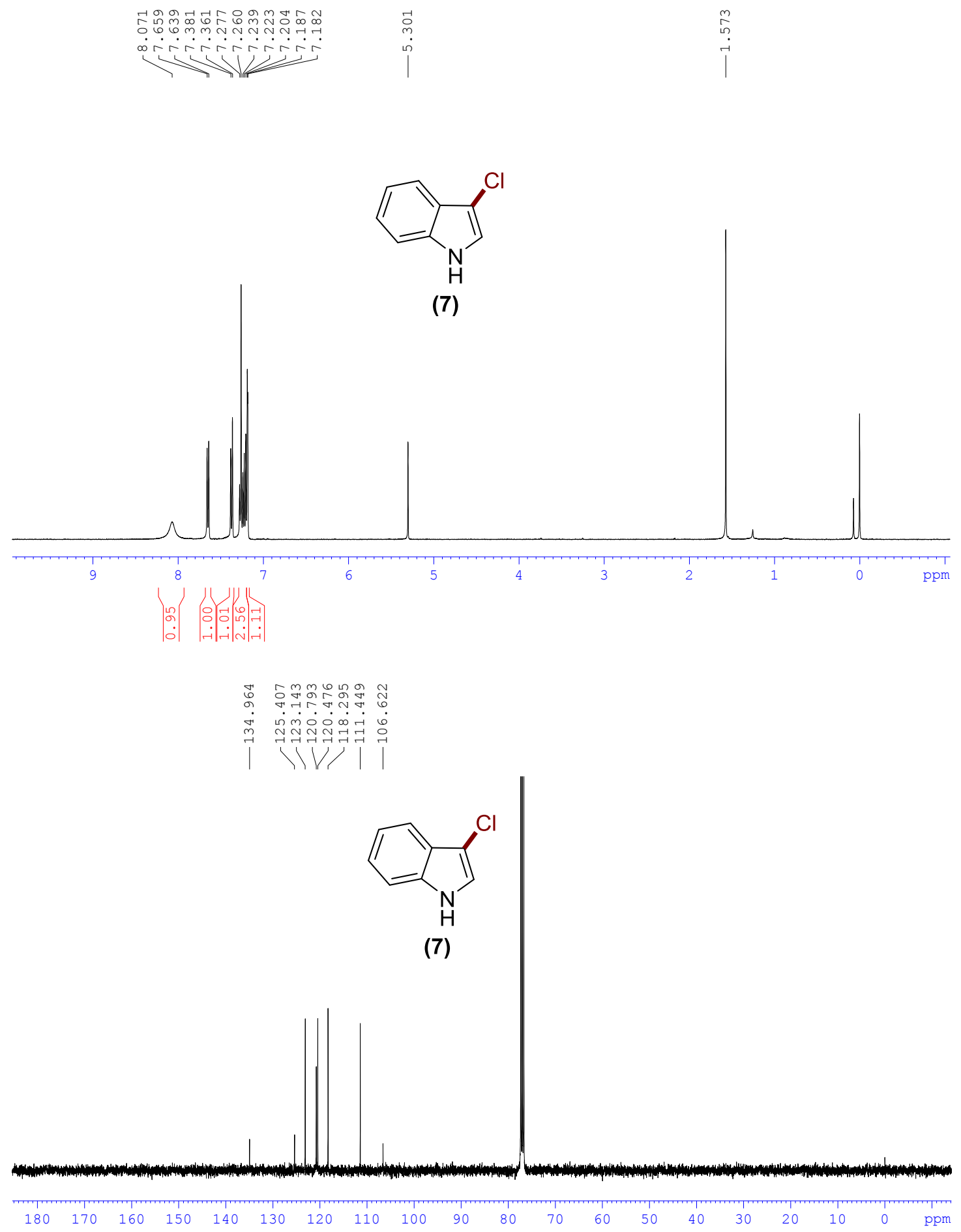




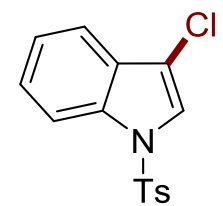

(8)
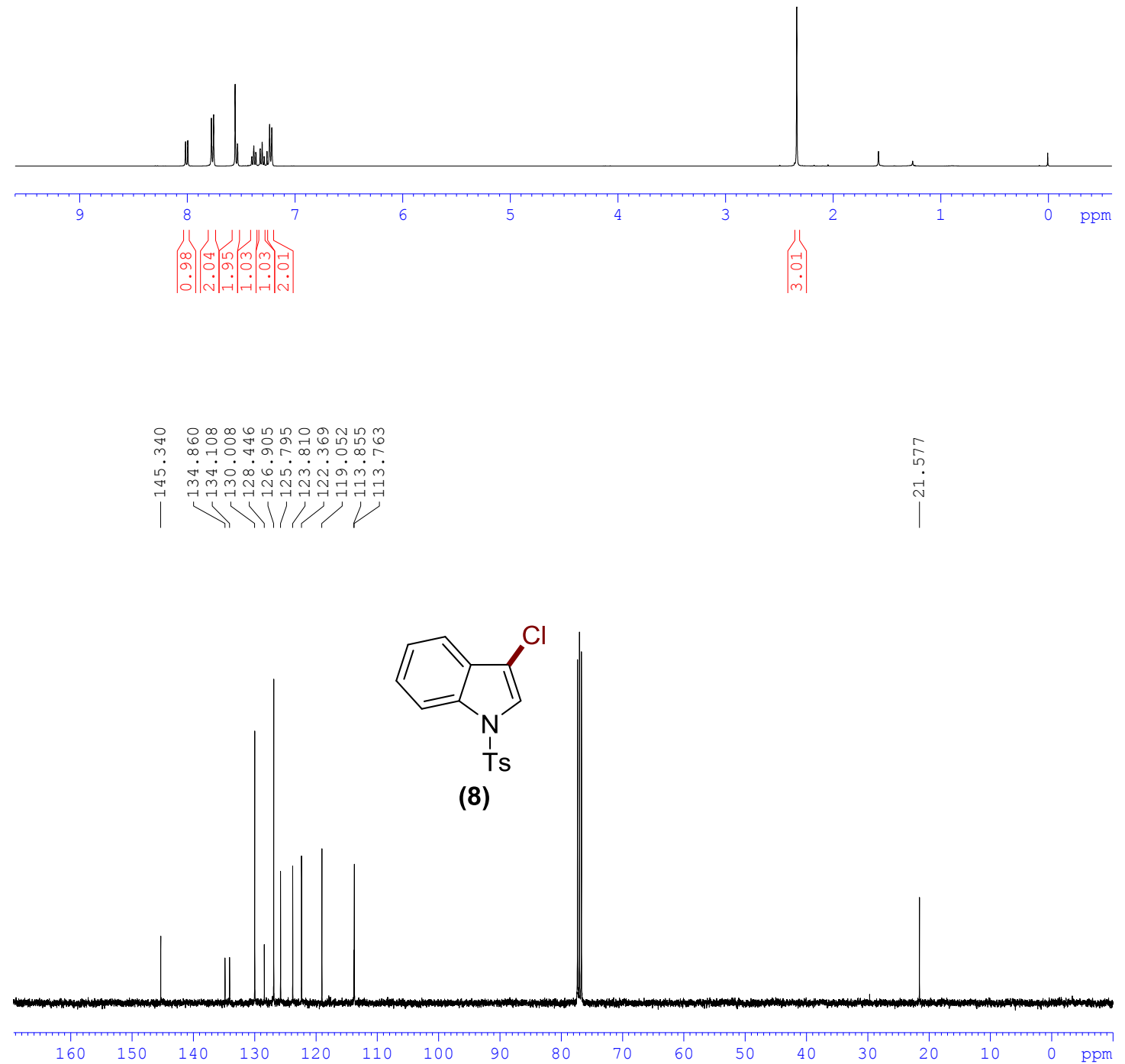


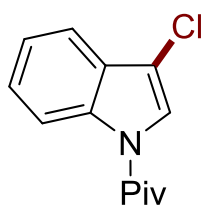

(9)
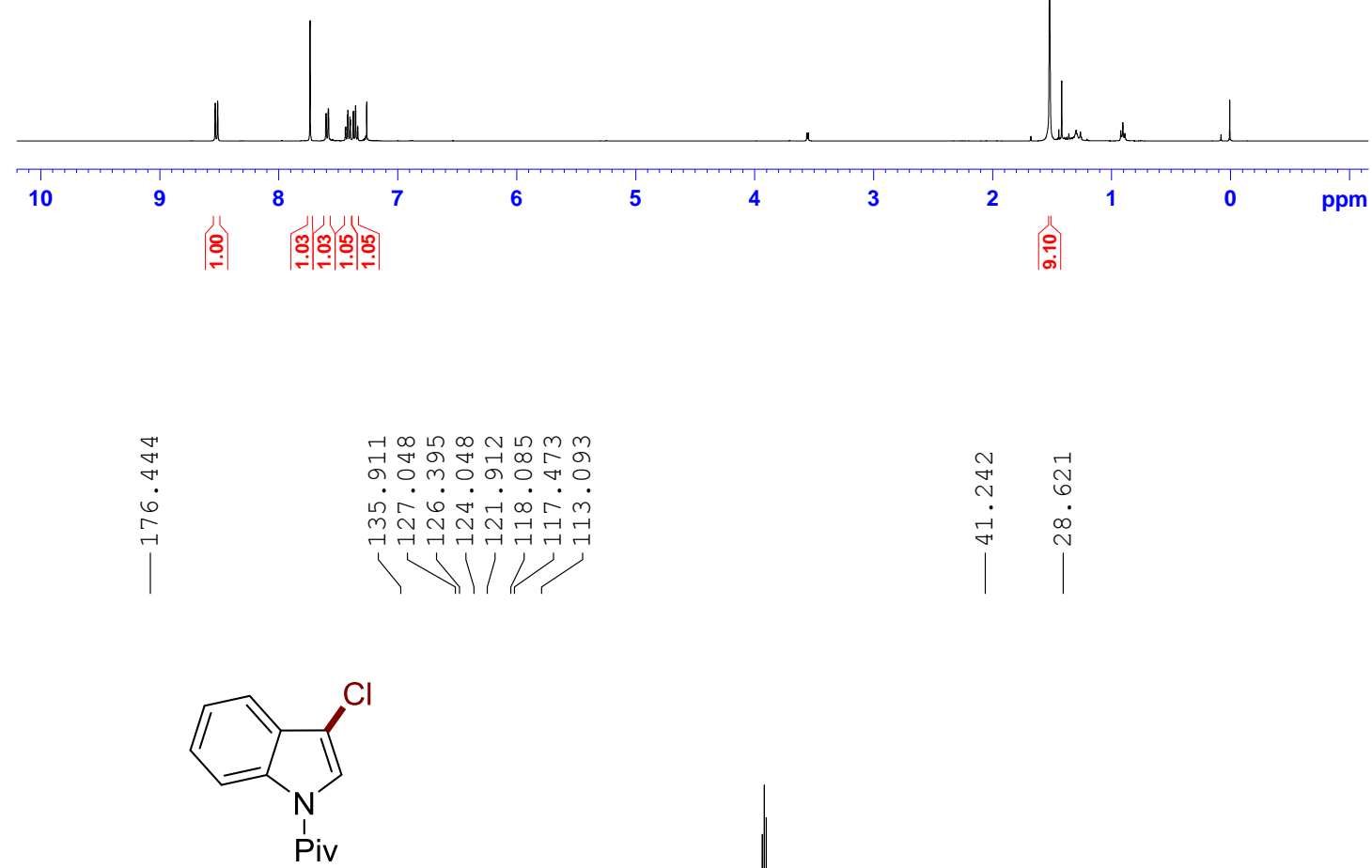

(9)

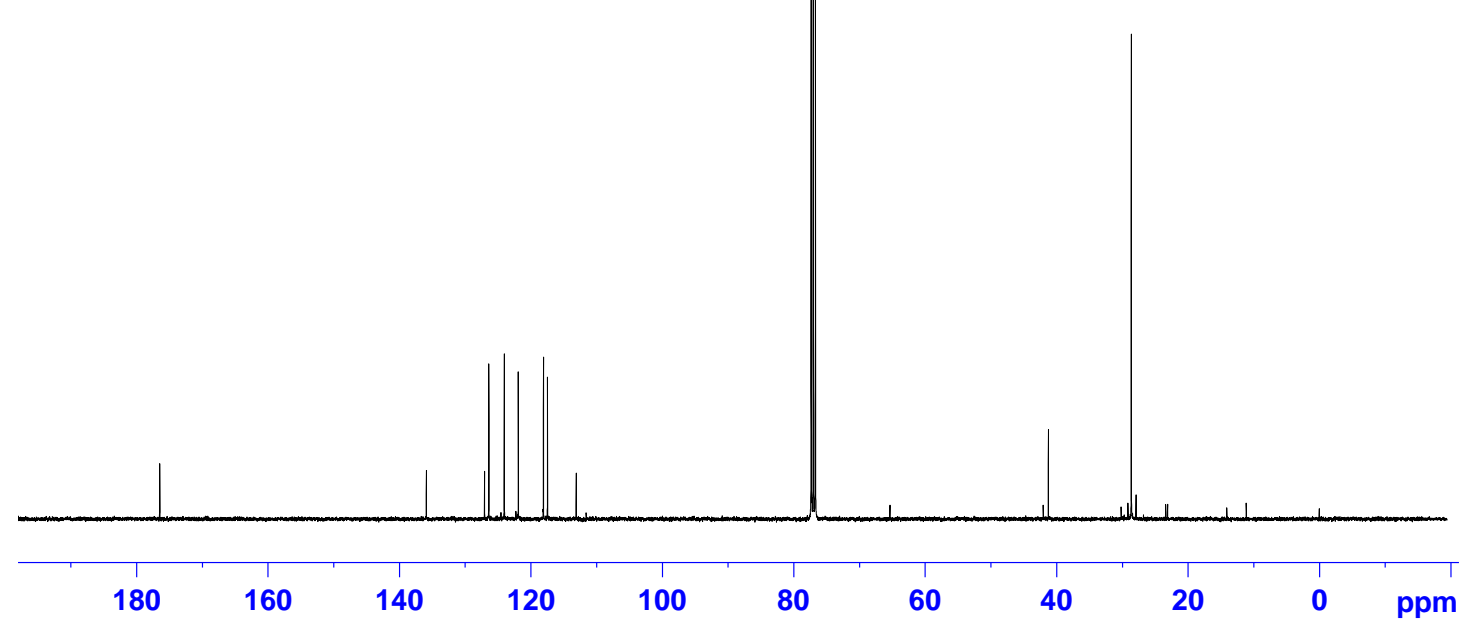




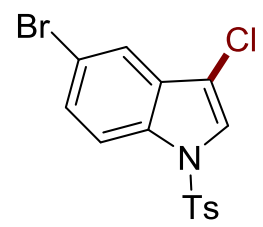

(10)
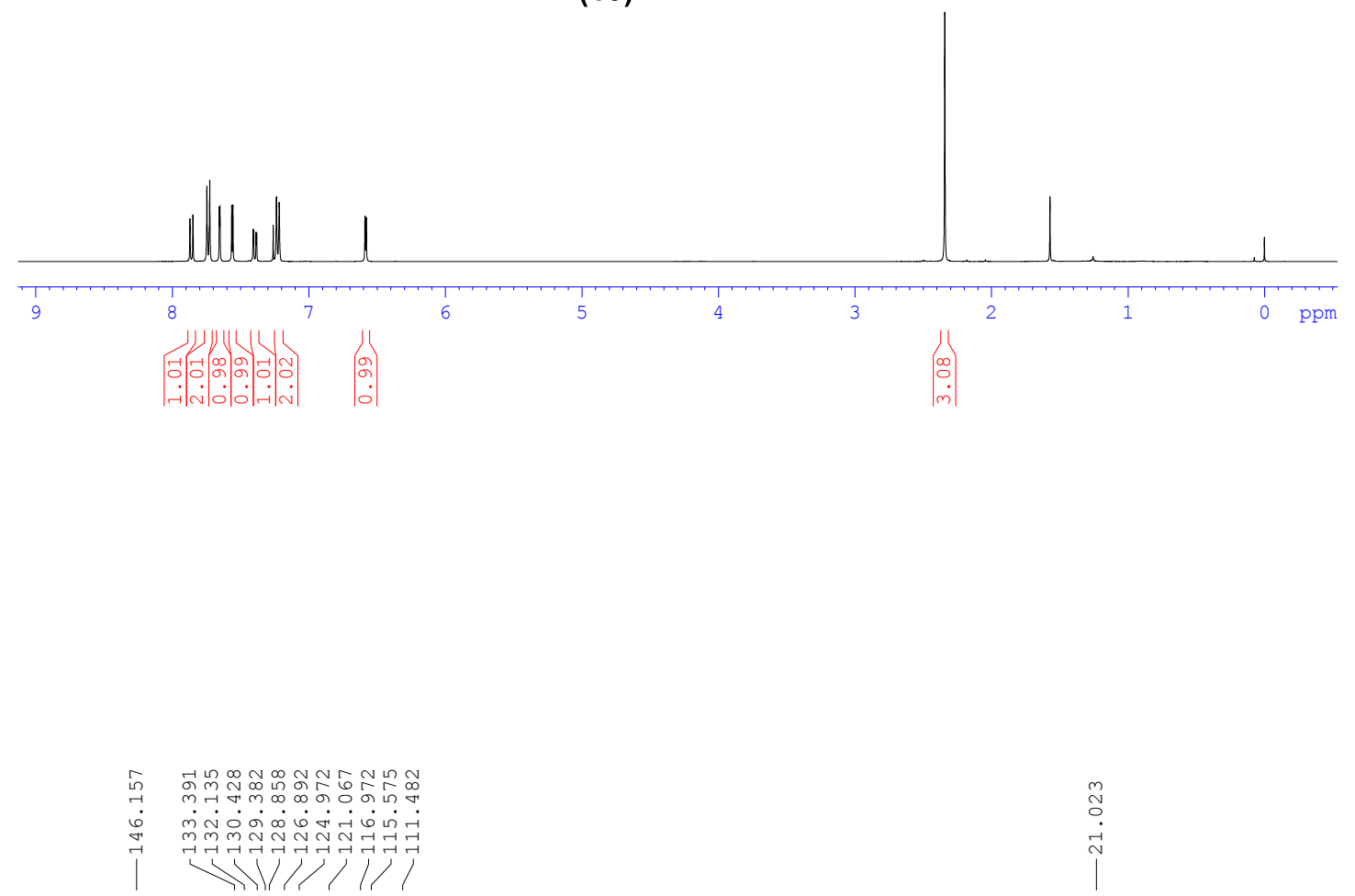

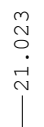

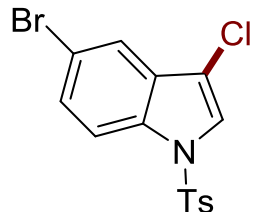

(10)

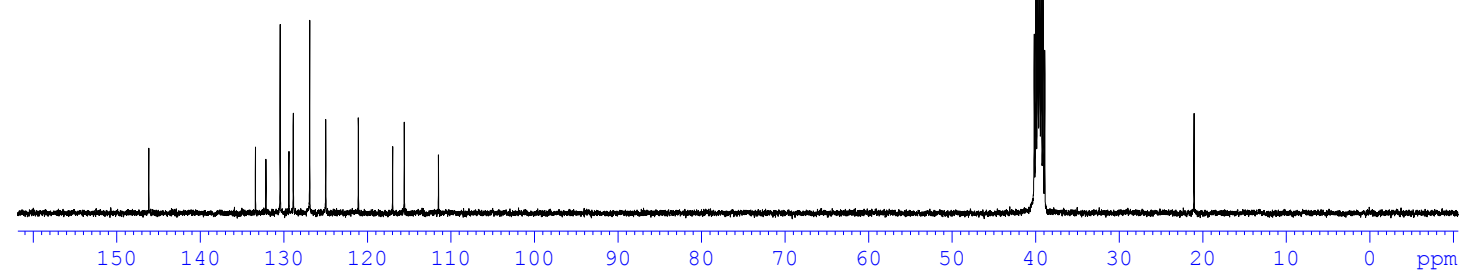




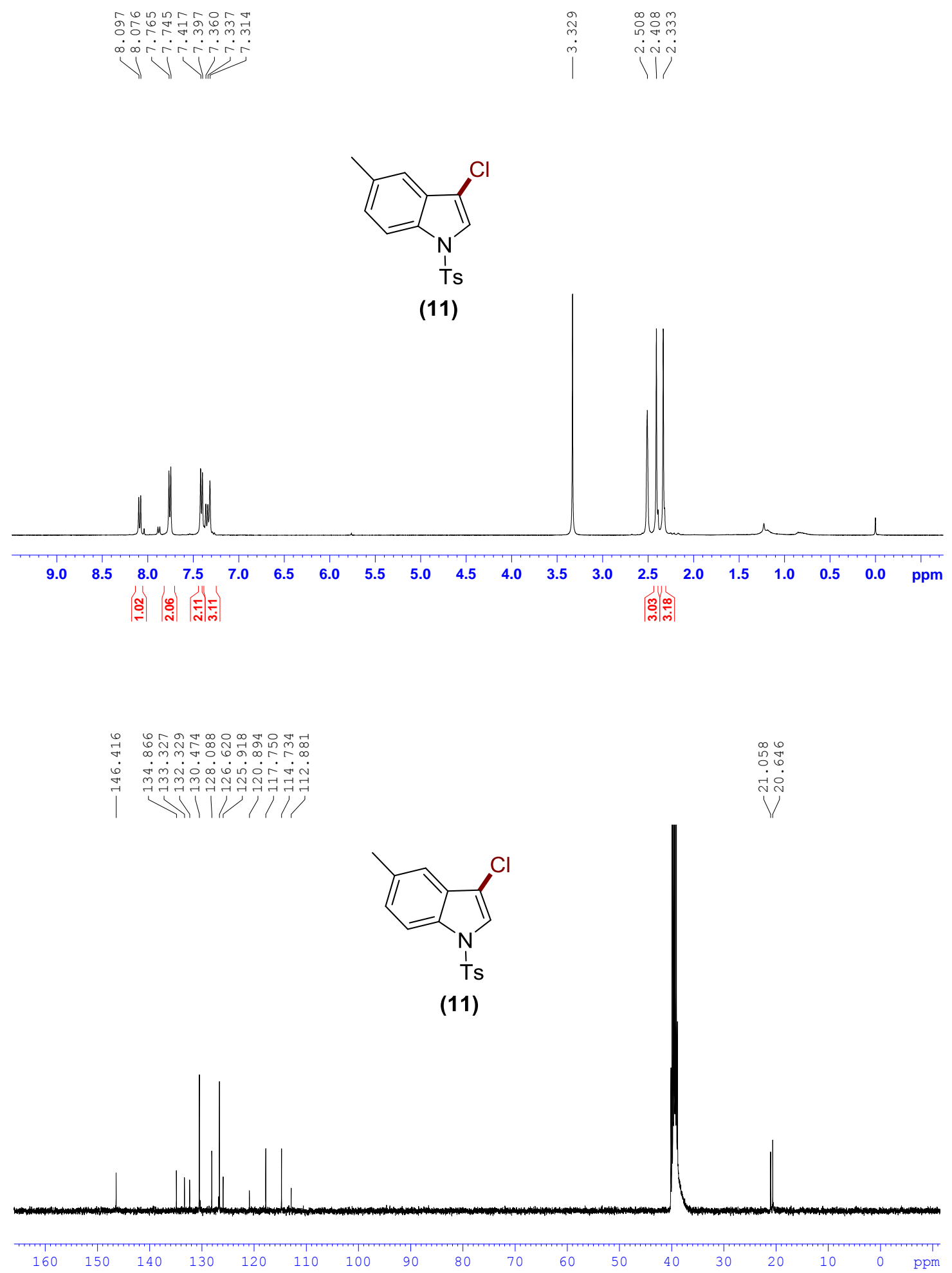




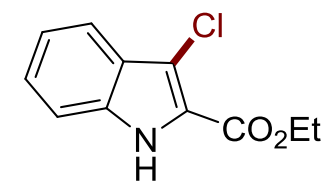

(12)
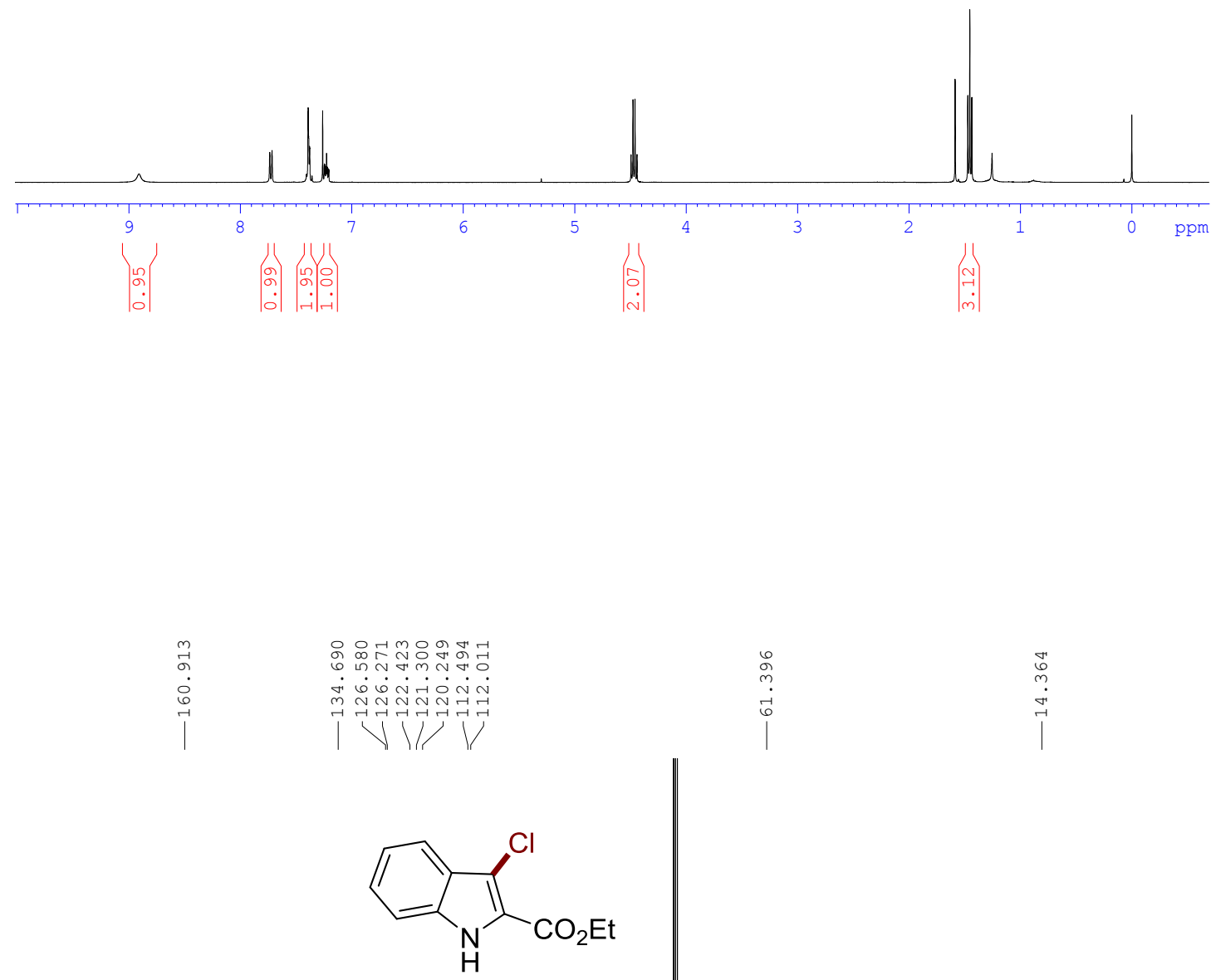

(12)

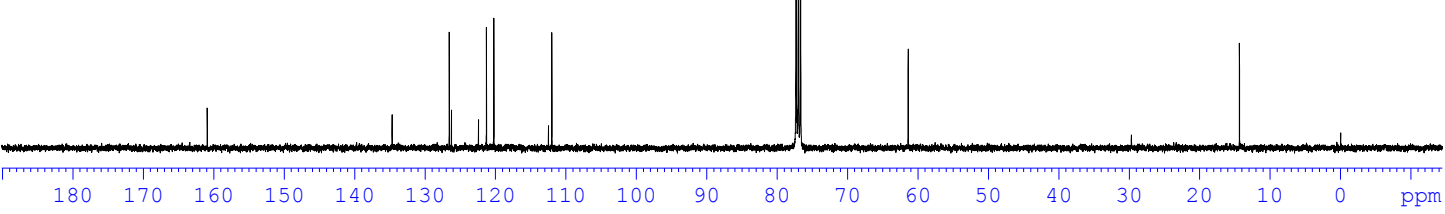




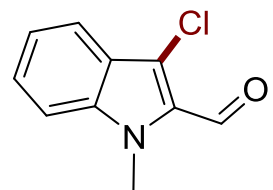

(13)
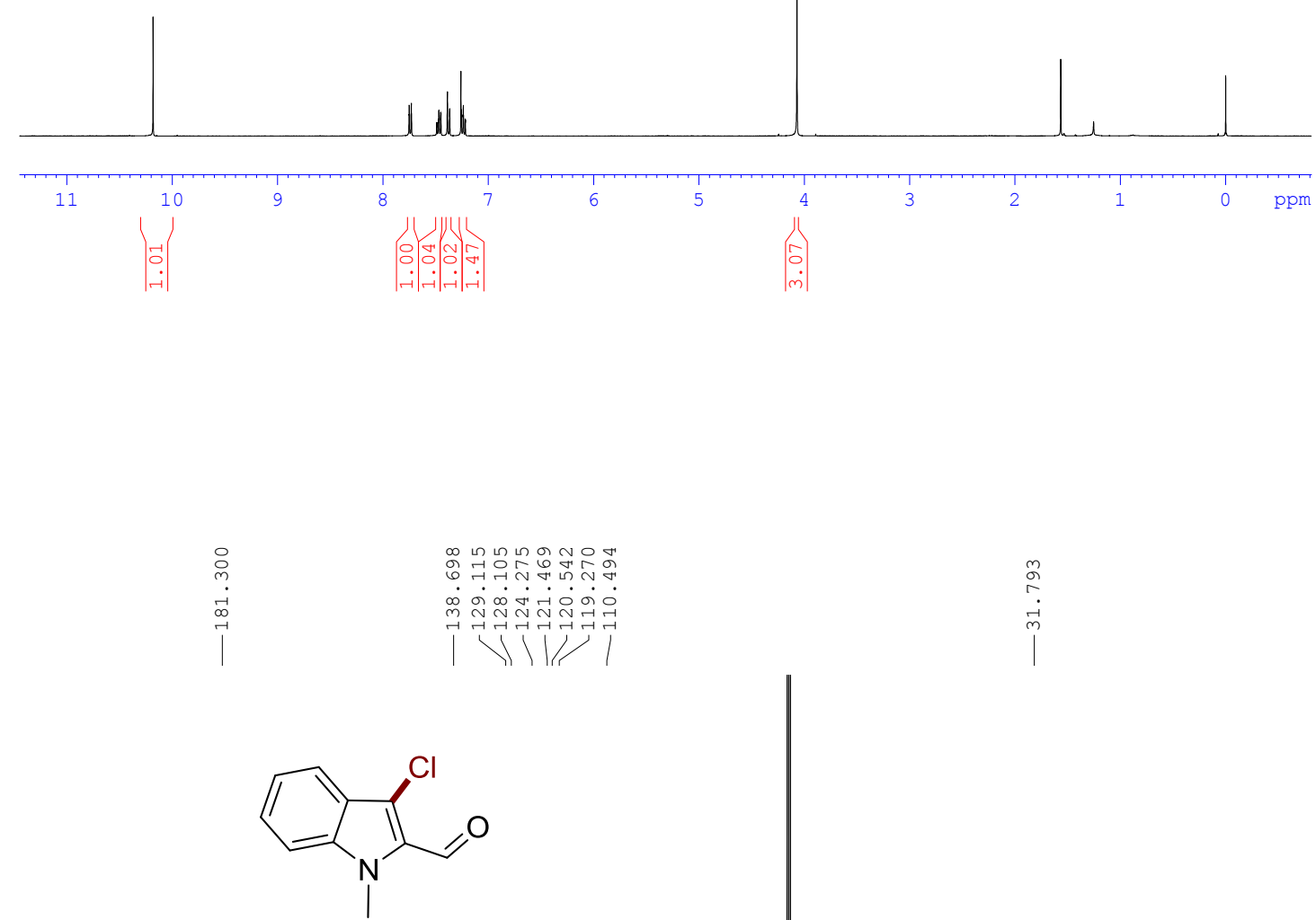

(13)

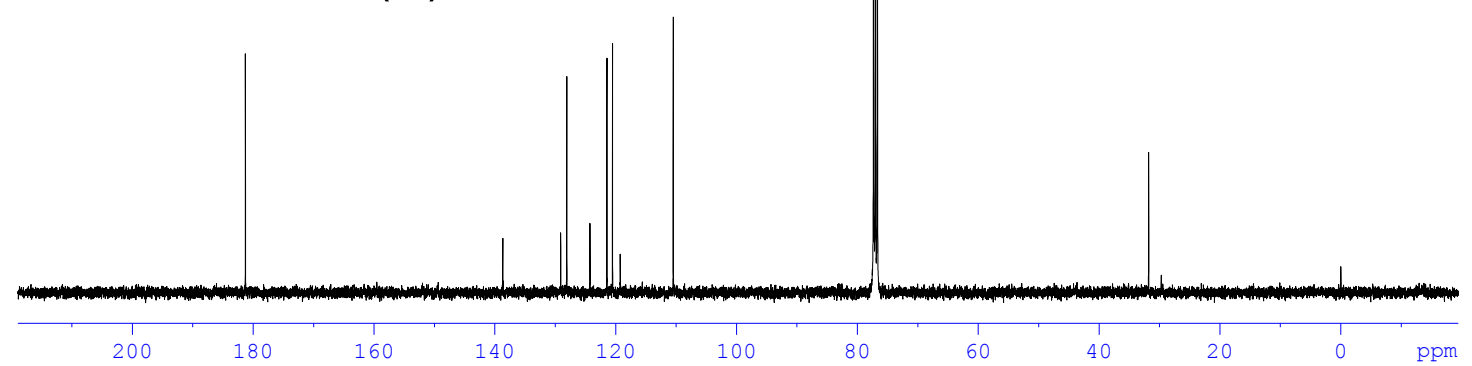




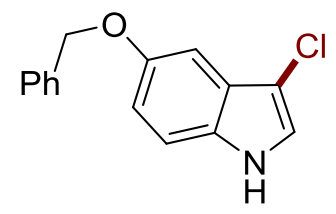

(14)
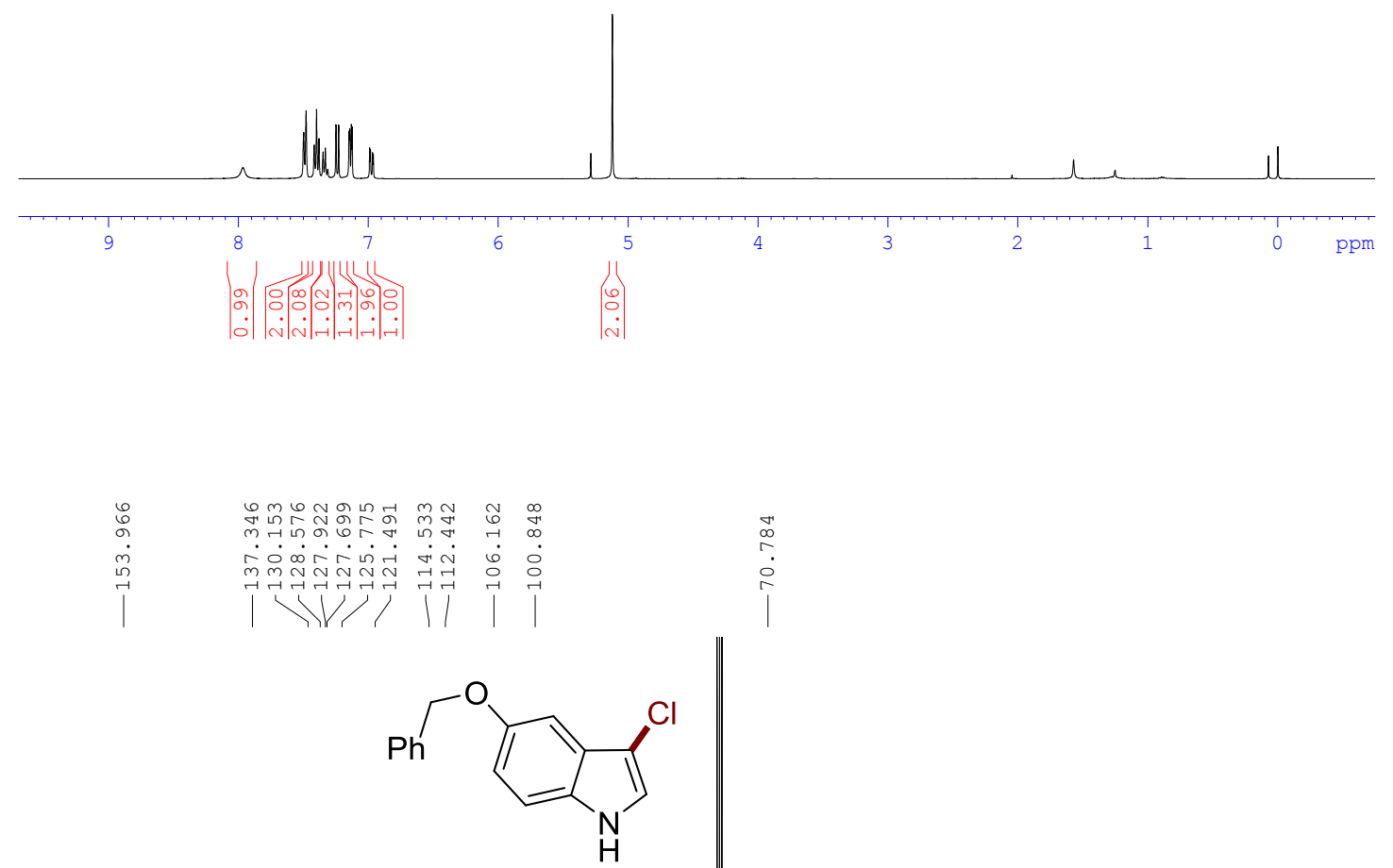

(14)

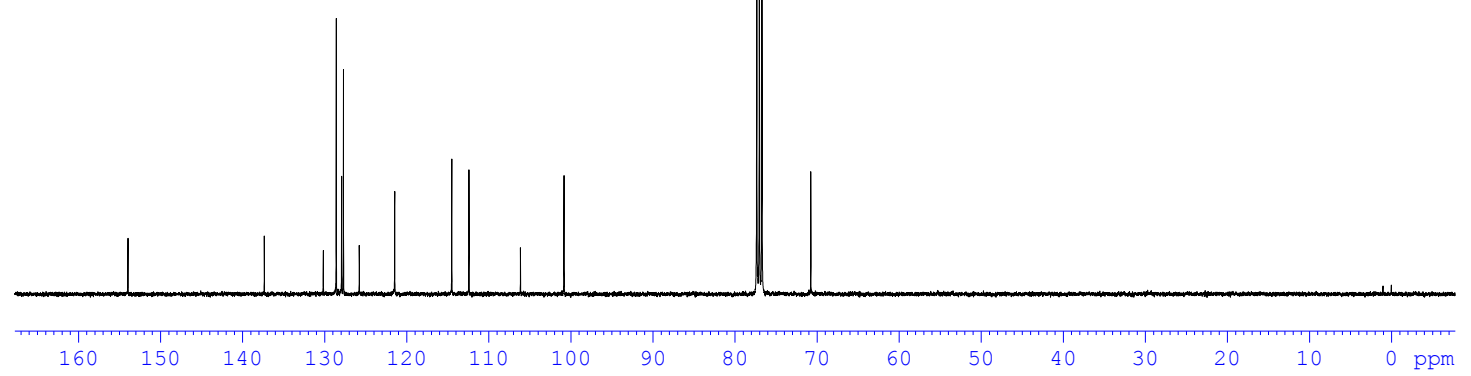




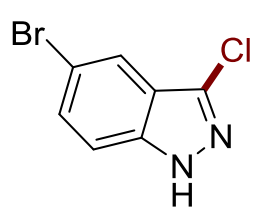

(15)
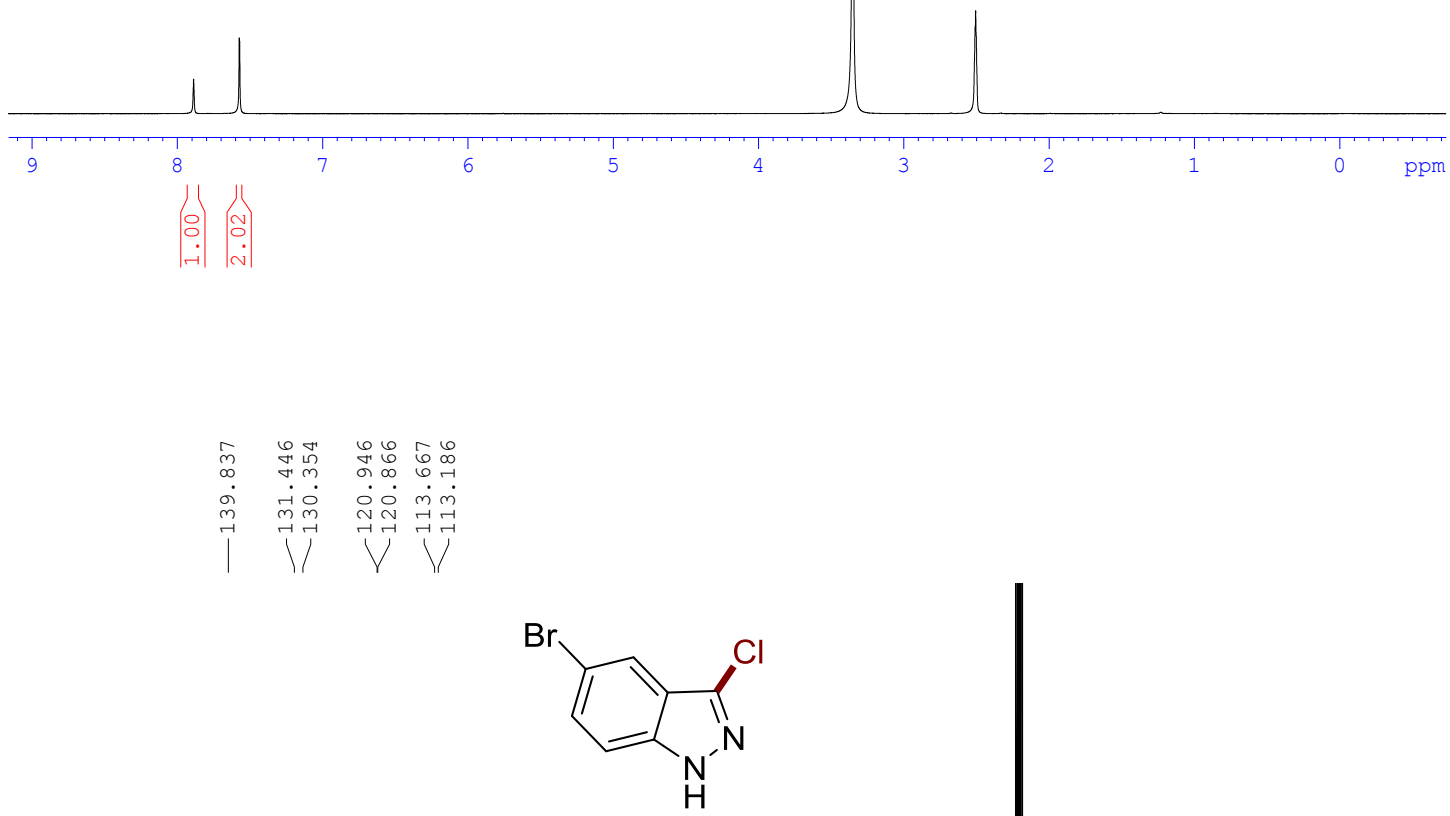

(15)

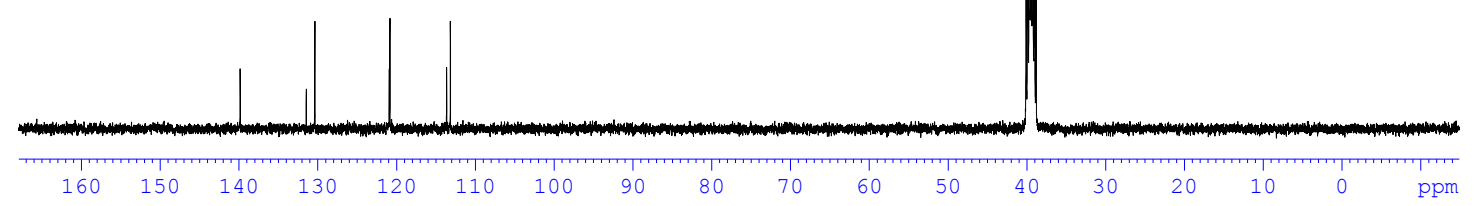




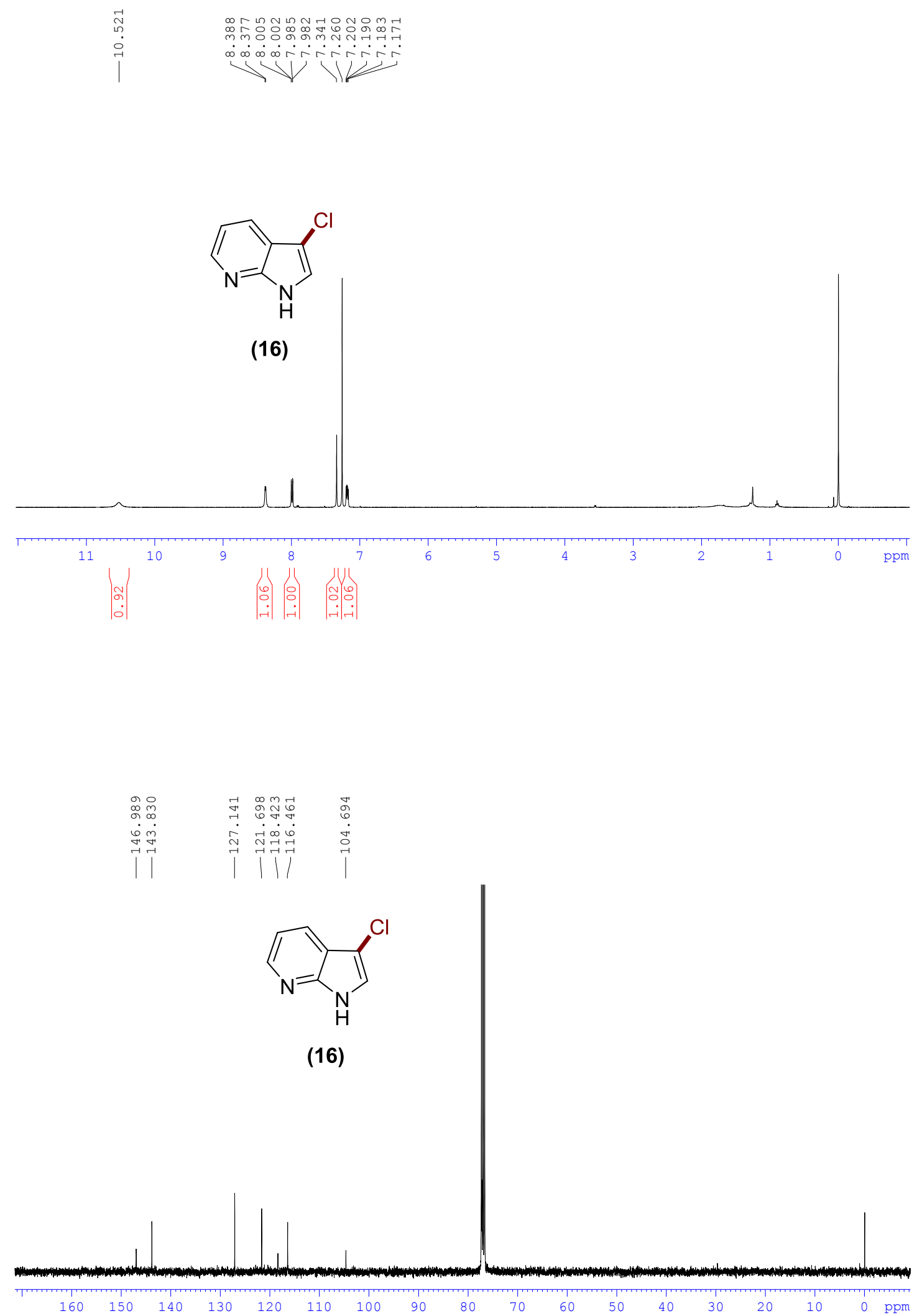




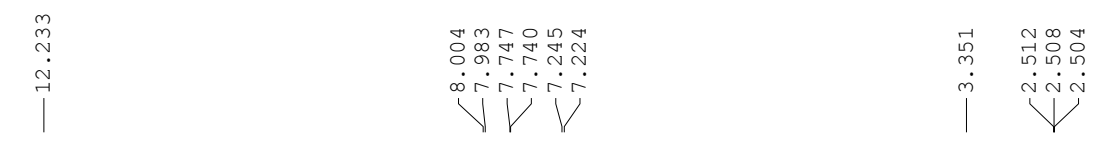<smiles>Clc1ccc2c(Cl)c[nH]c2n1</smiles>

(17)

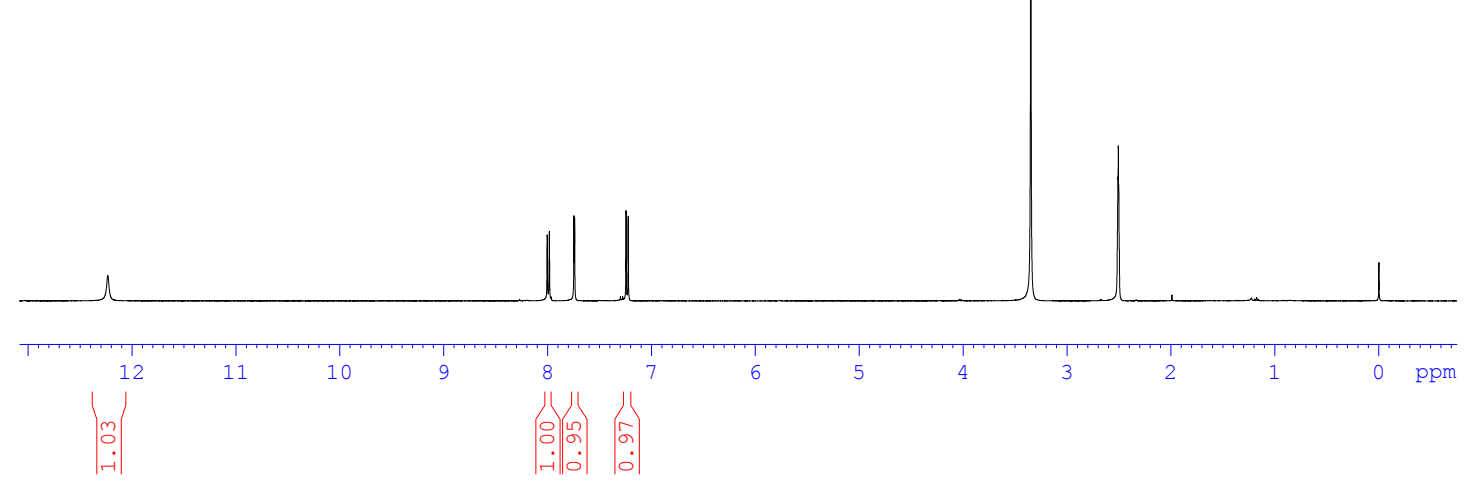

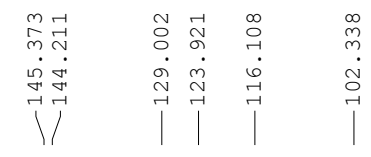<smiles>Clc1ccc2c(Cl)c[nH]c2n1</smiles>

(17)

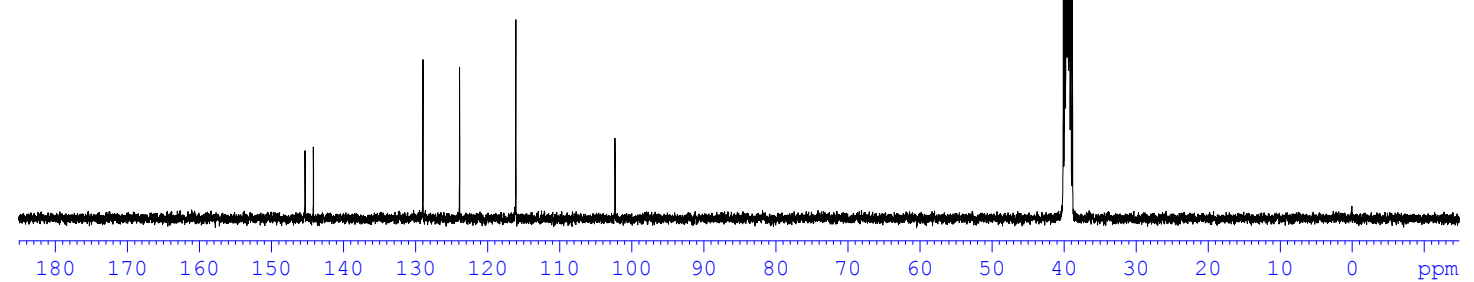




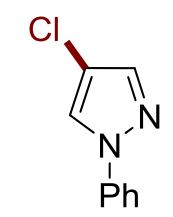

(18)
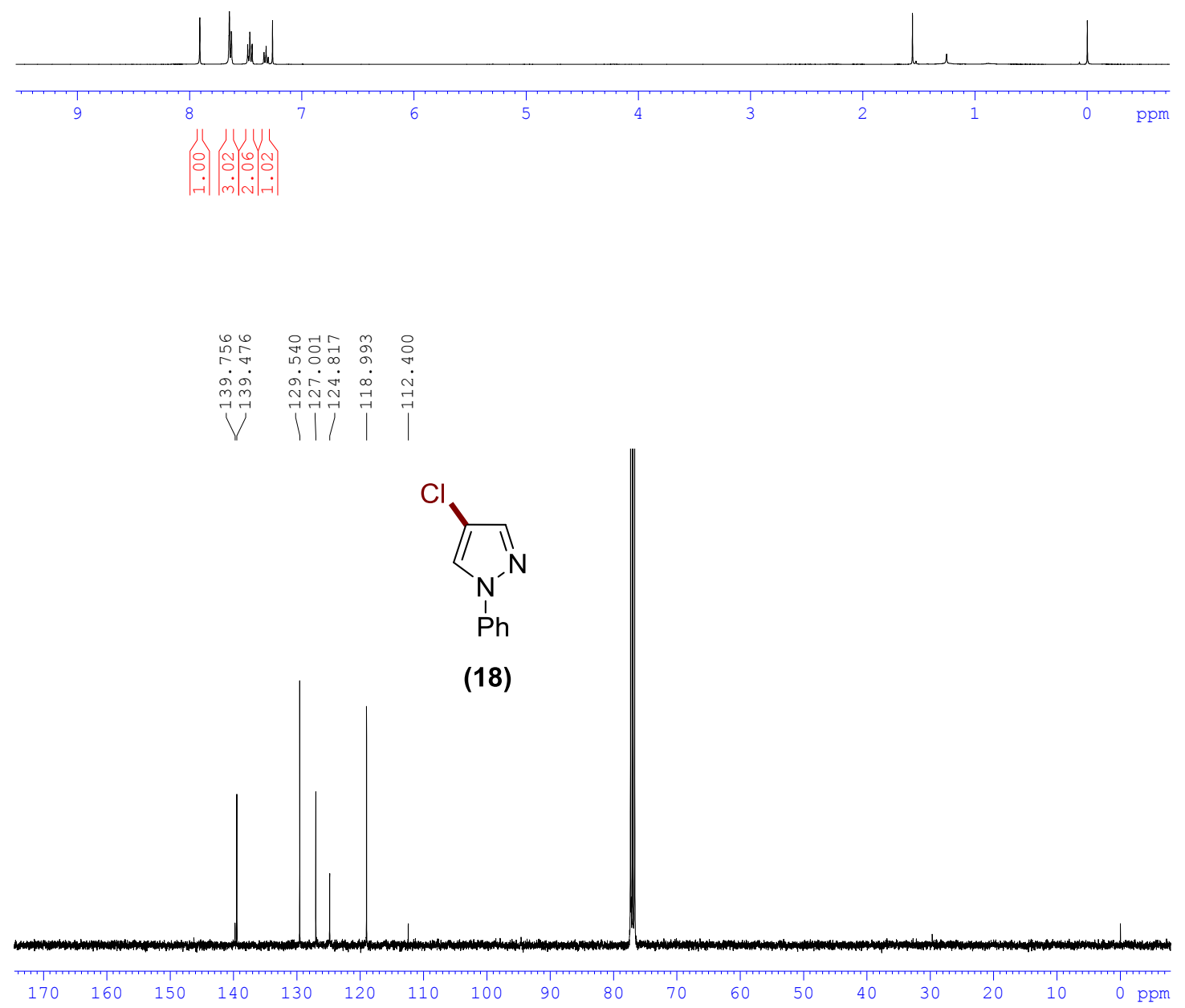

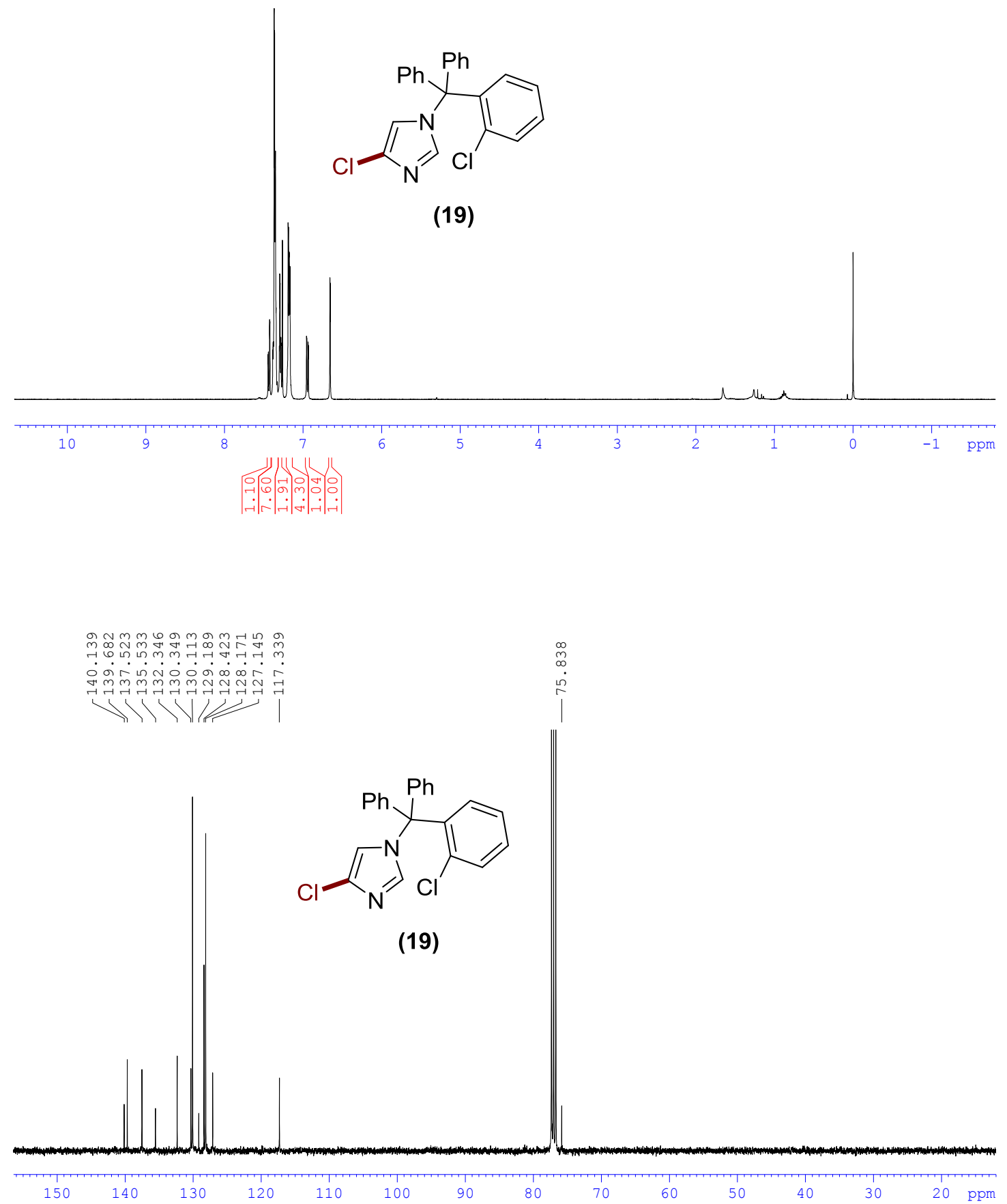
<smiles>Clc1nc(Cl)c2c(Cl)c[nH]c2n1</smiles>

(20)
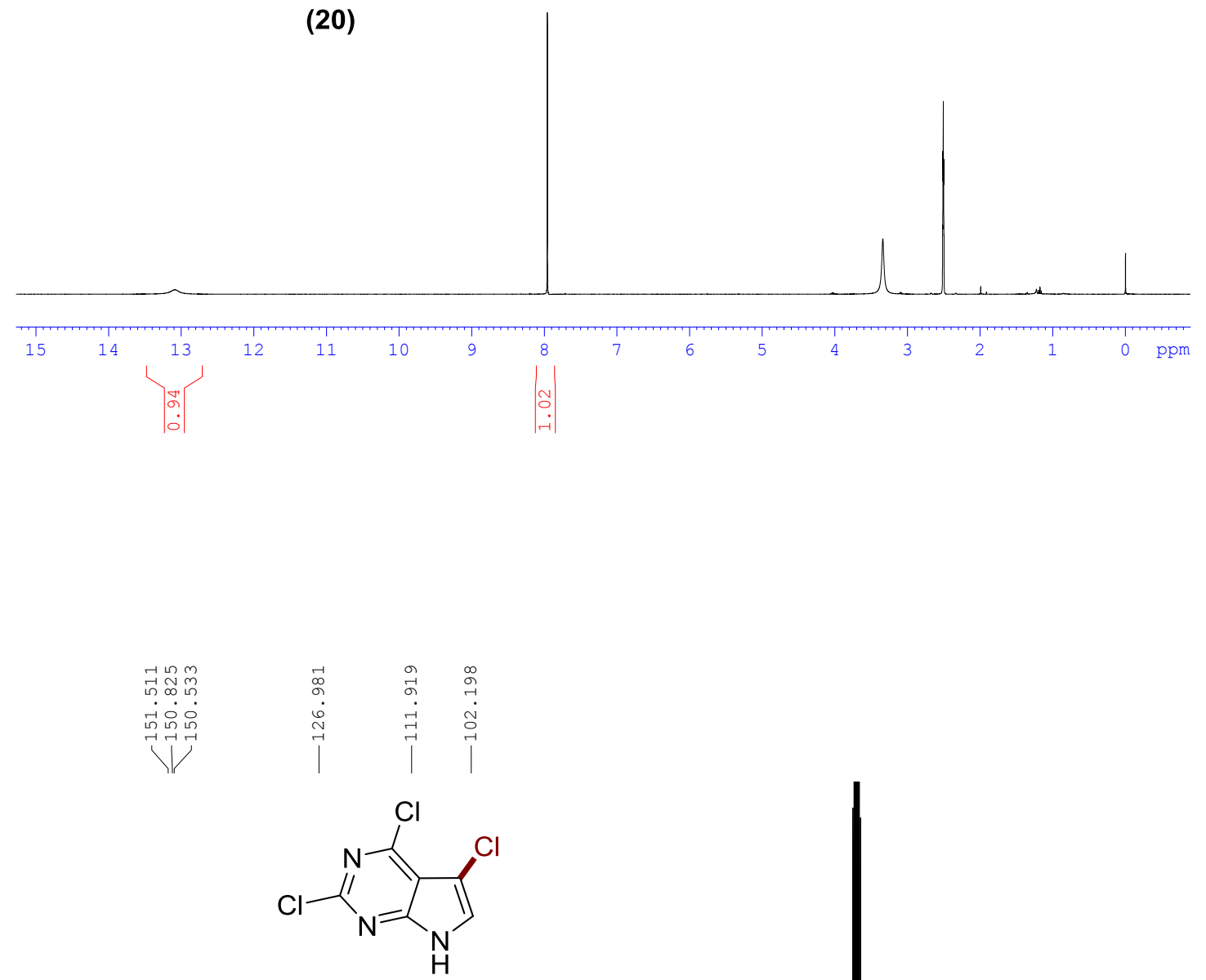

(20)

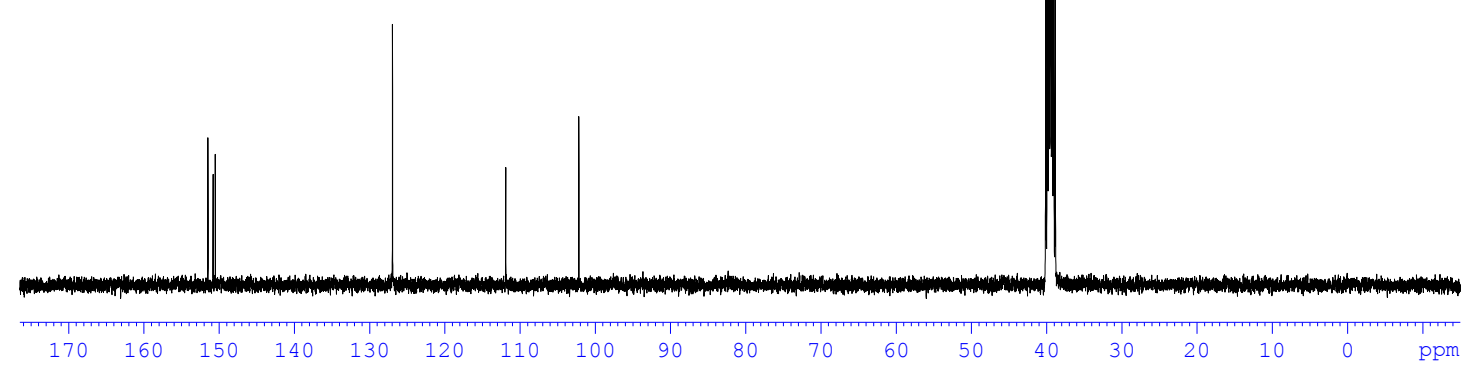



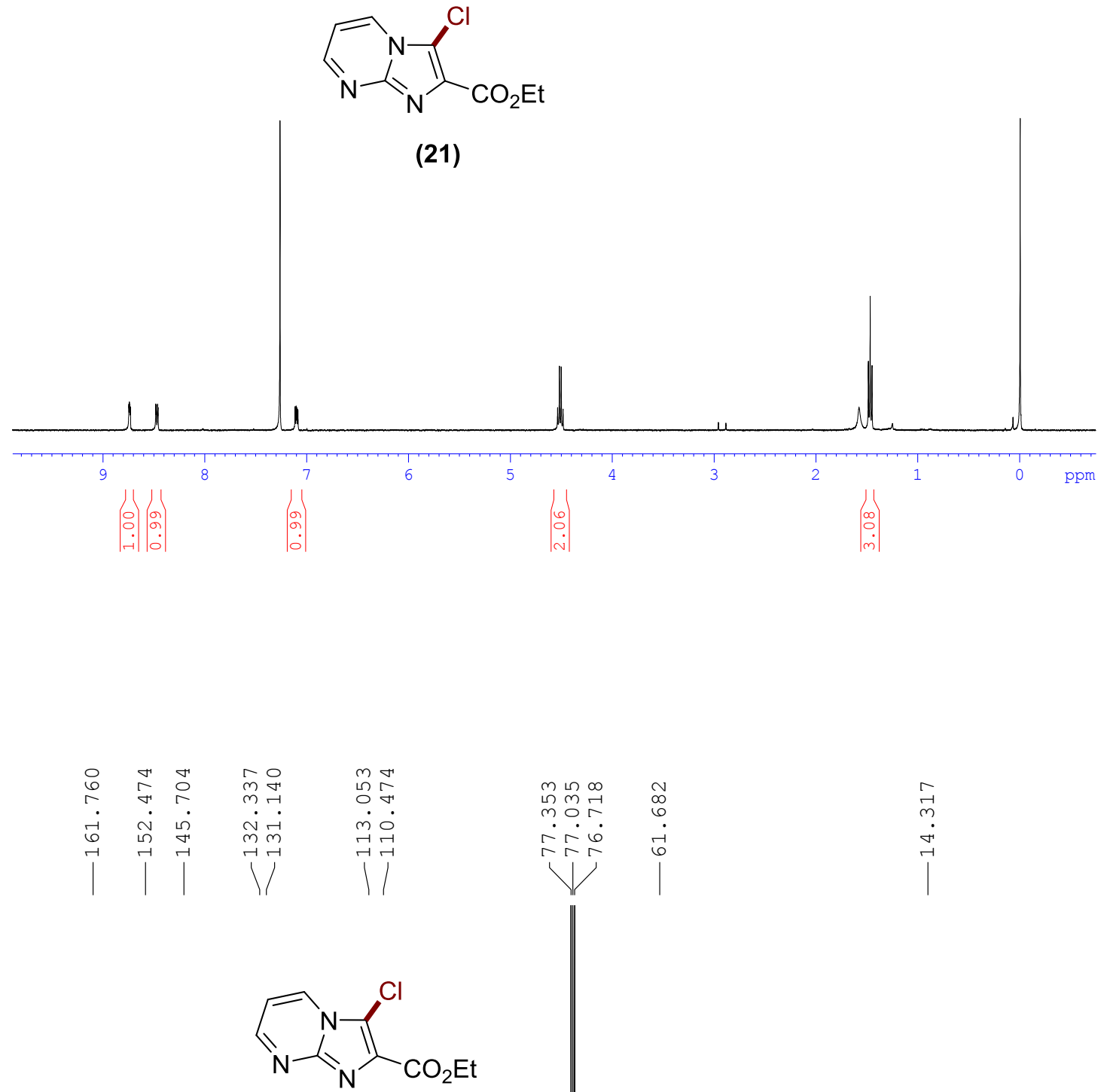

(21)

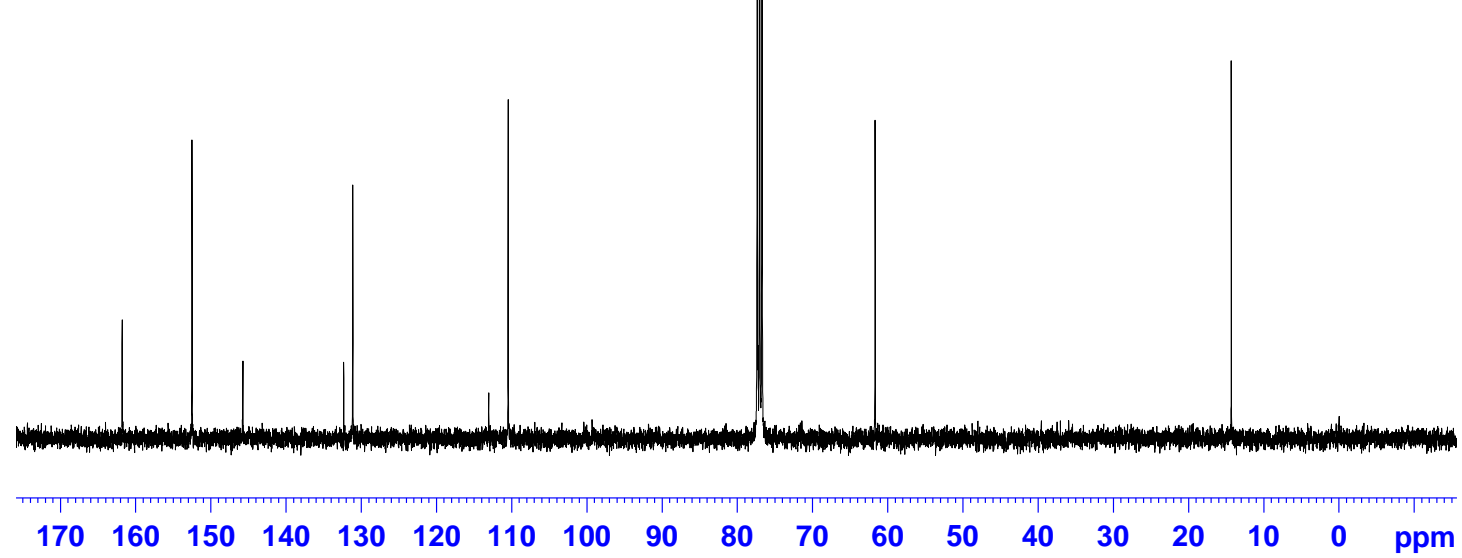




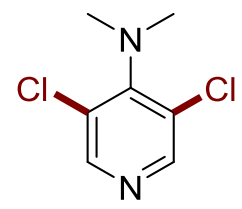

(22)
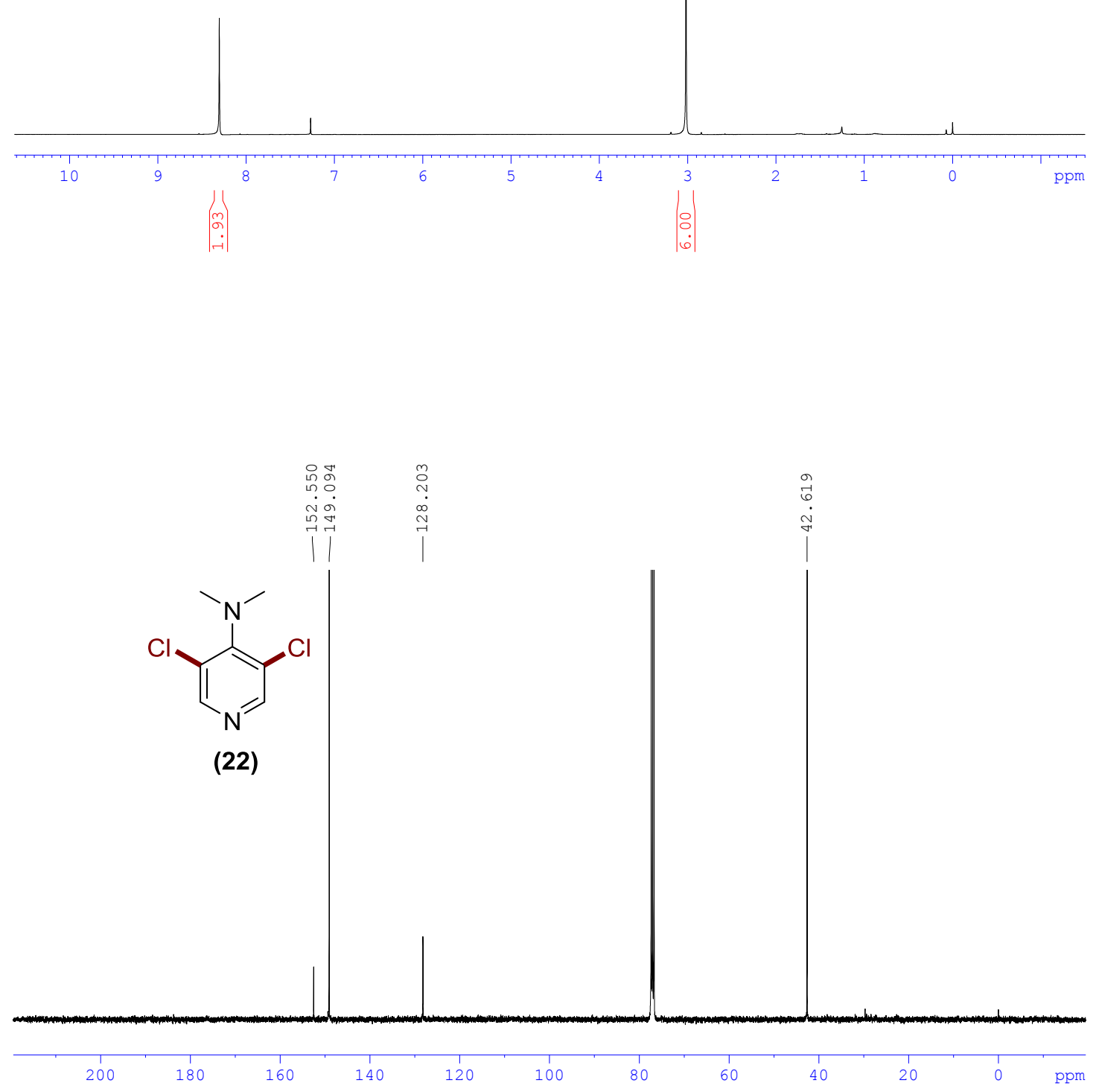


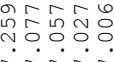<smiles>Nc1ccc(Cl)nc1Cl</smiles>

(23)
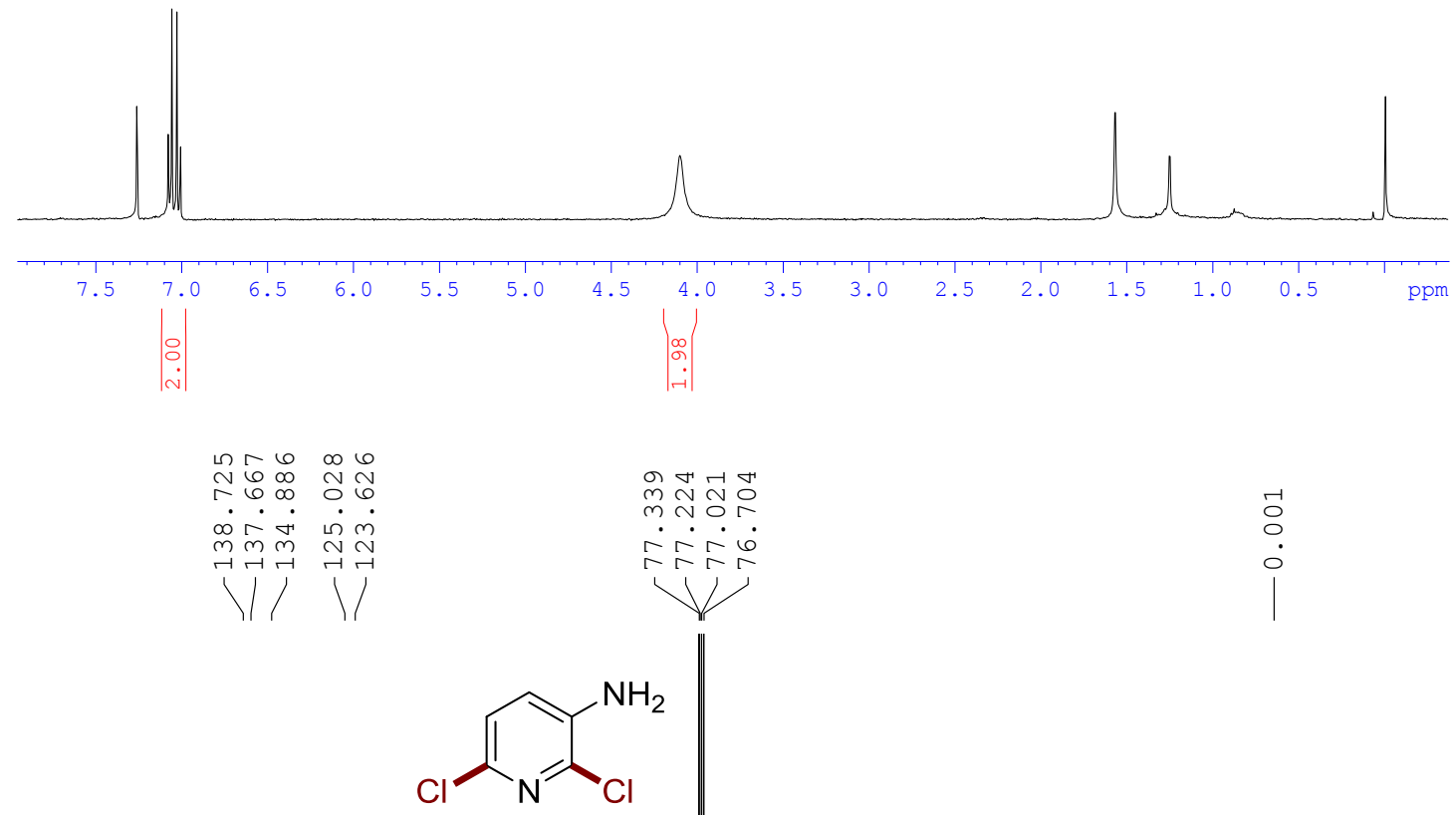

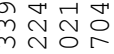

도은

W

ㅇ.

(23)

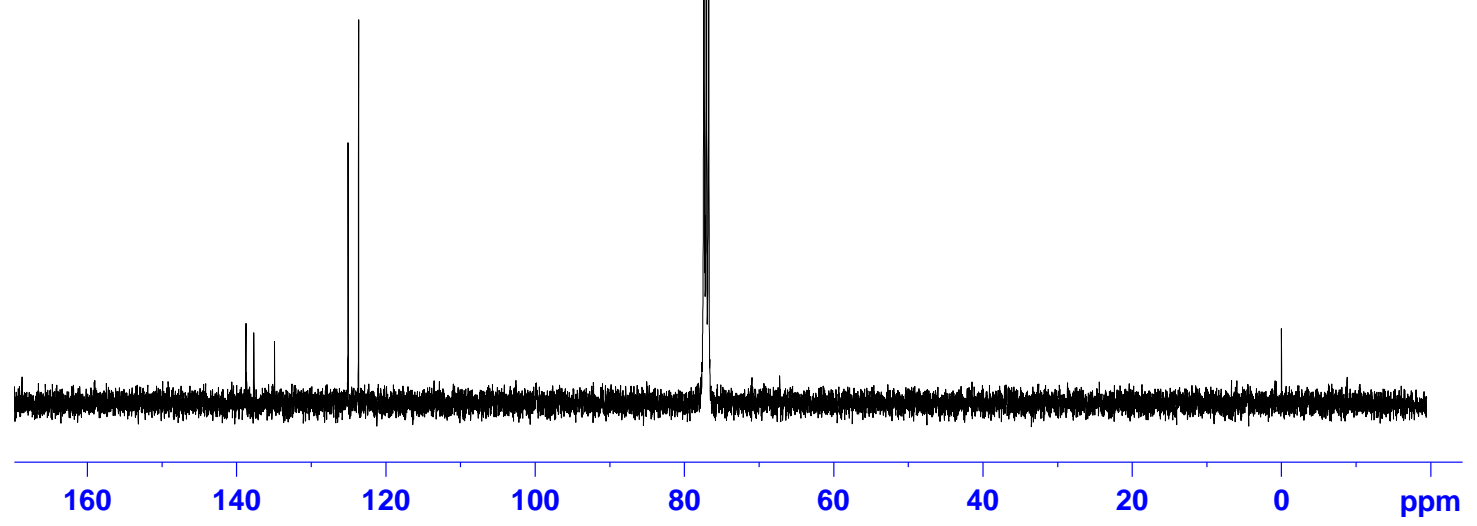




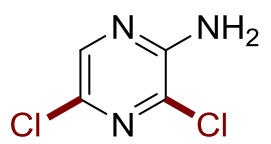

(24)

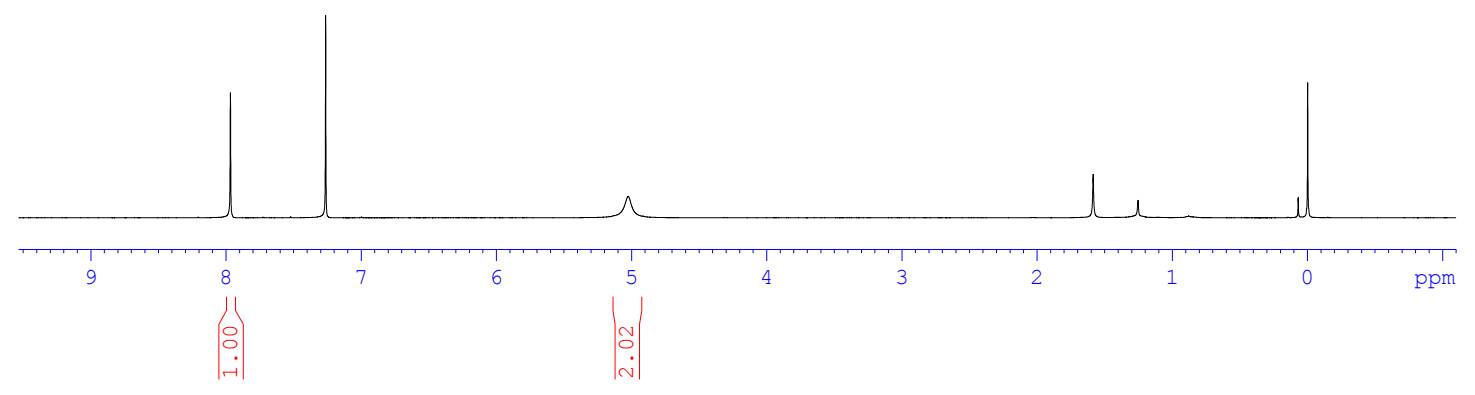

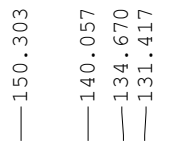

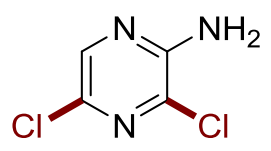

(24)

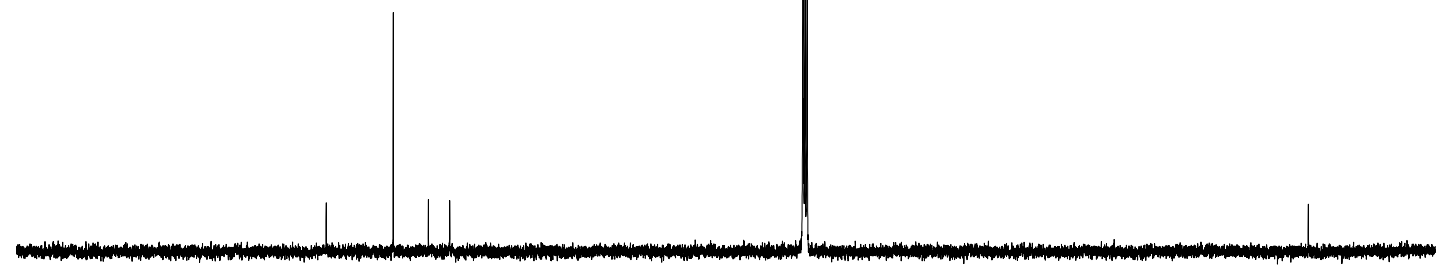

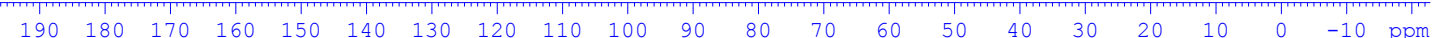


<smiles>ClNc1ccc2ccccc2n1</smiles>

(25)
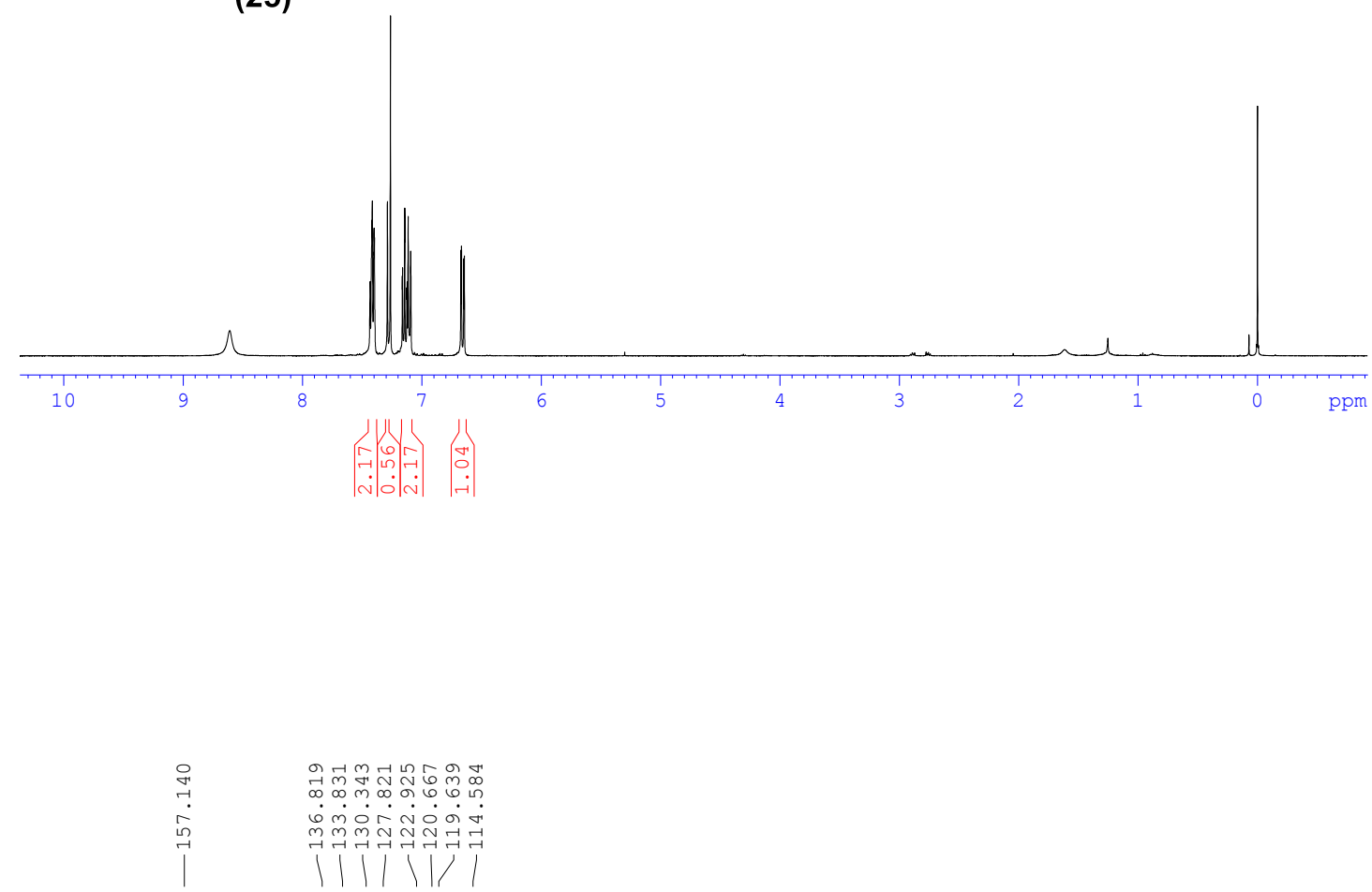<smiles>ClNc1ccc2ccccc2n1</smiles>

(25)

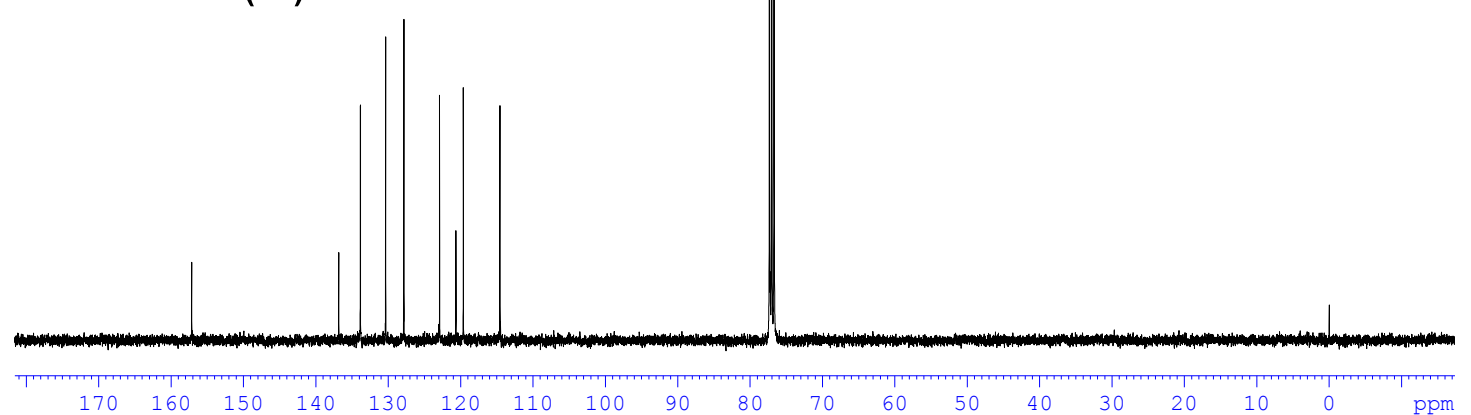


<smiles>Nc1cnc2ccccc2c1Cl</smiles>

(26)

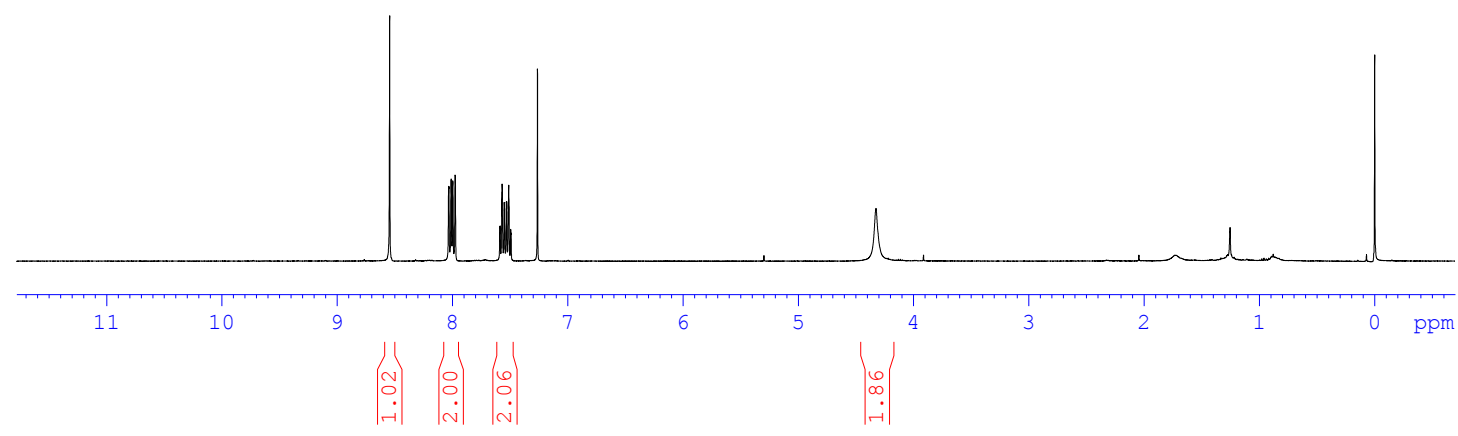

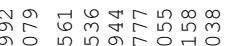

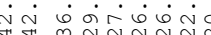

Th

|<smiles>Nc1cnc2ccccc2c1Cl</smiles>

(26)

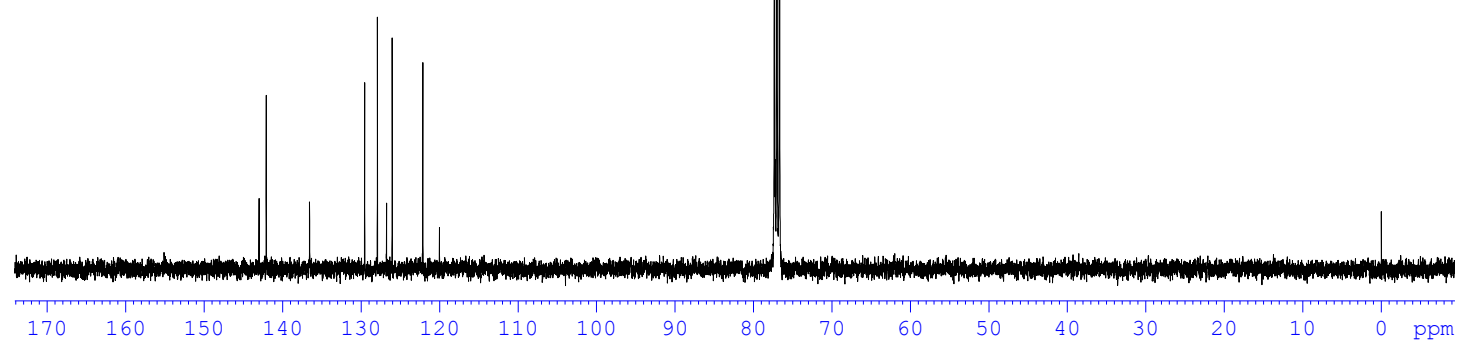


<smiles>Nc1ncc2ccccc2c1Cl</smiles>

(27)

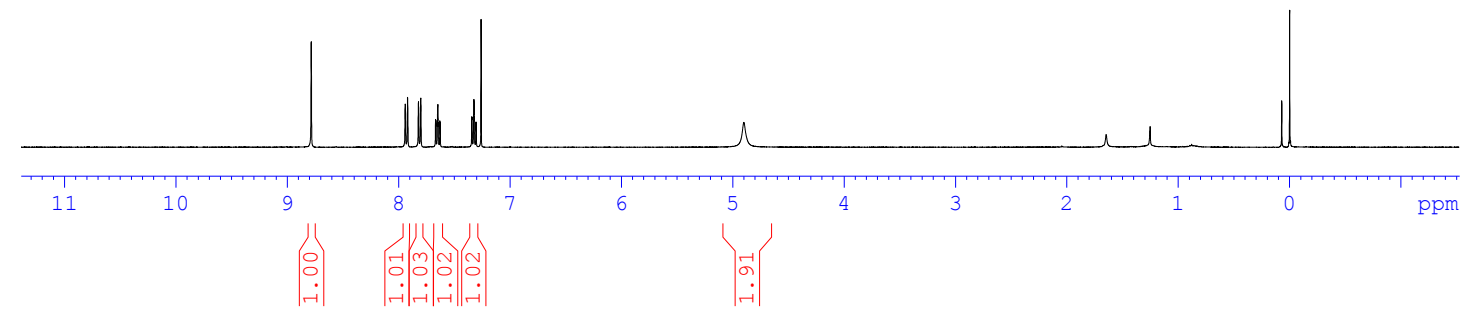

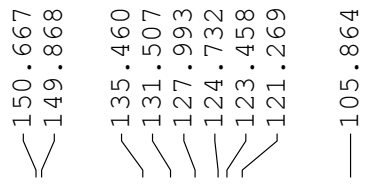<smiles>Nc1ncc2ccccc2c1Cl</smiles>

(27)

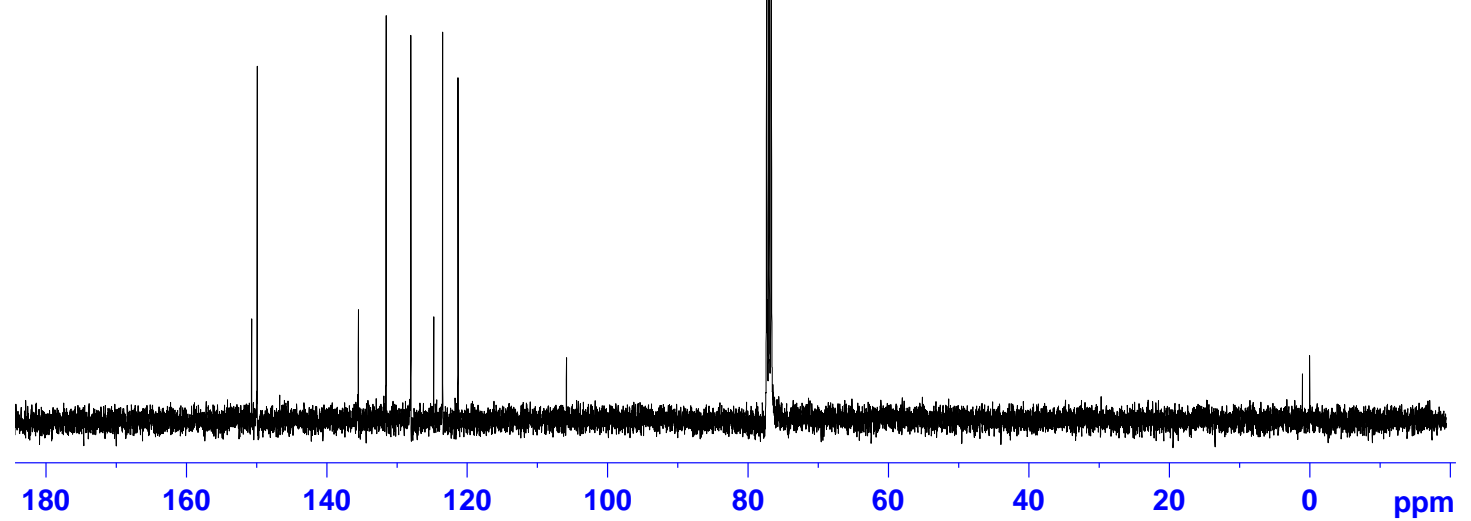


<smiles>CNc1ncc(Cl)cn1</smiles>

(28)
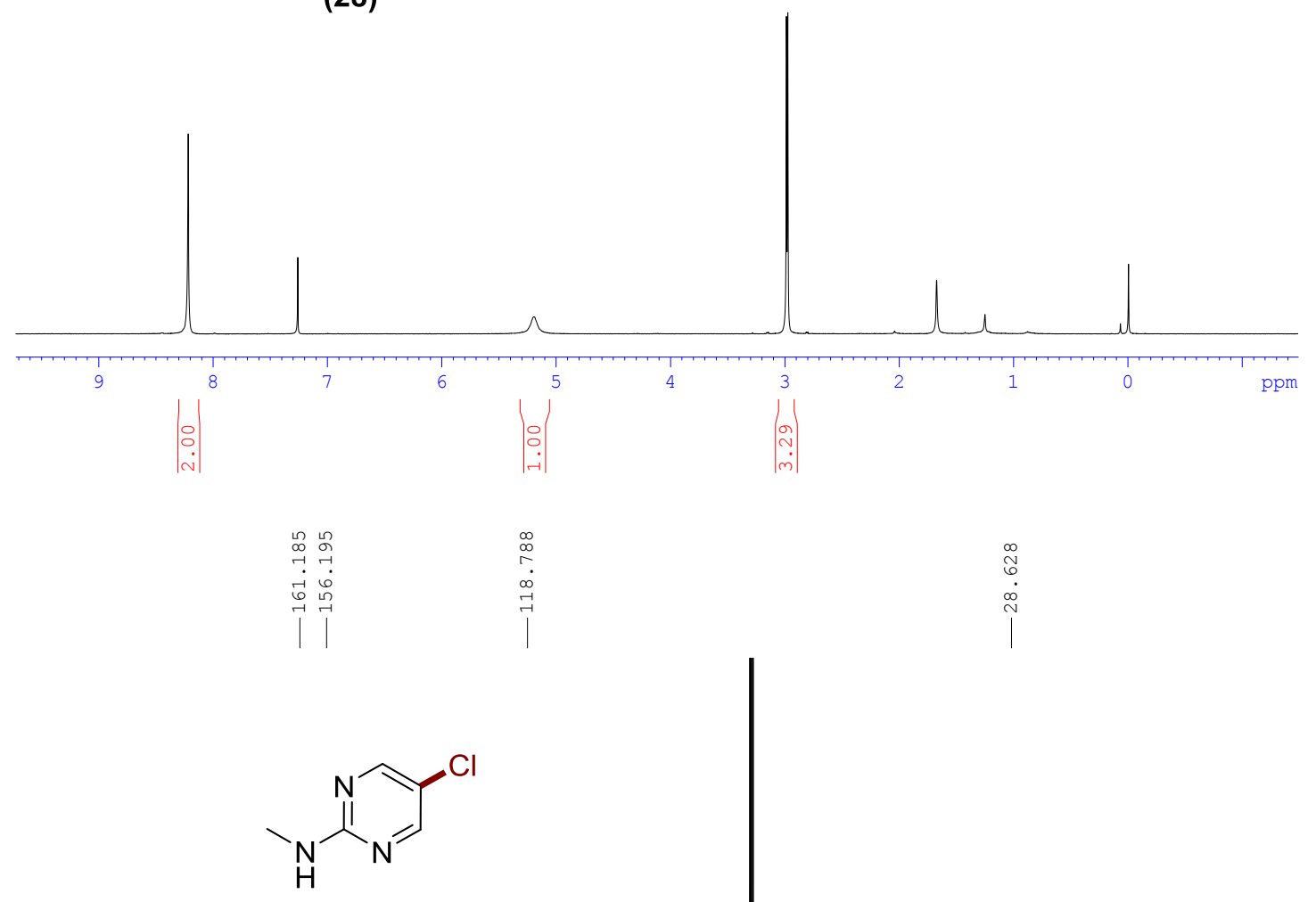

(28)

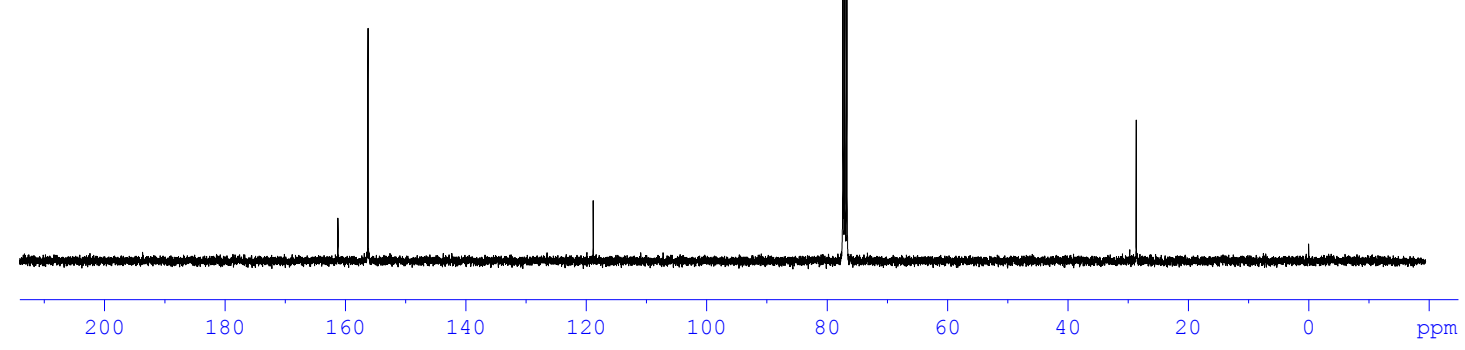




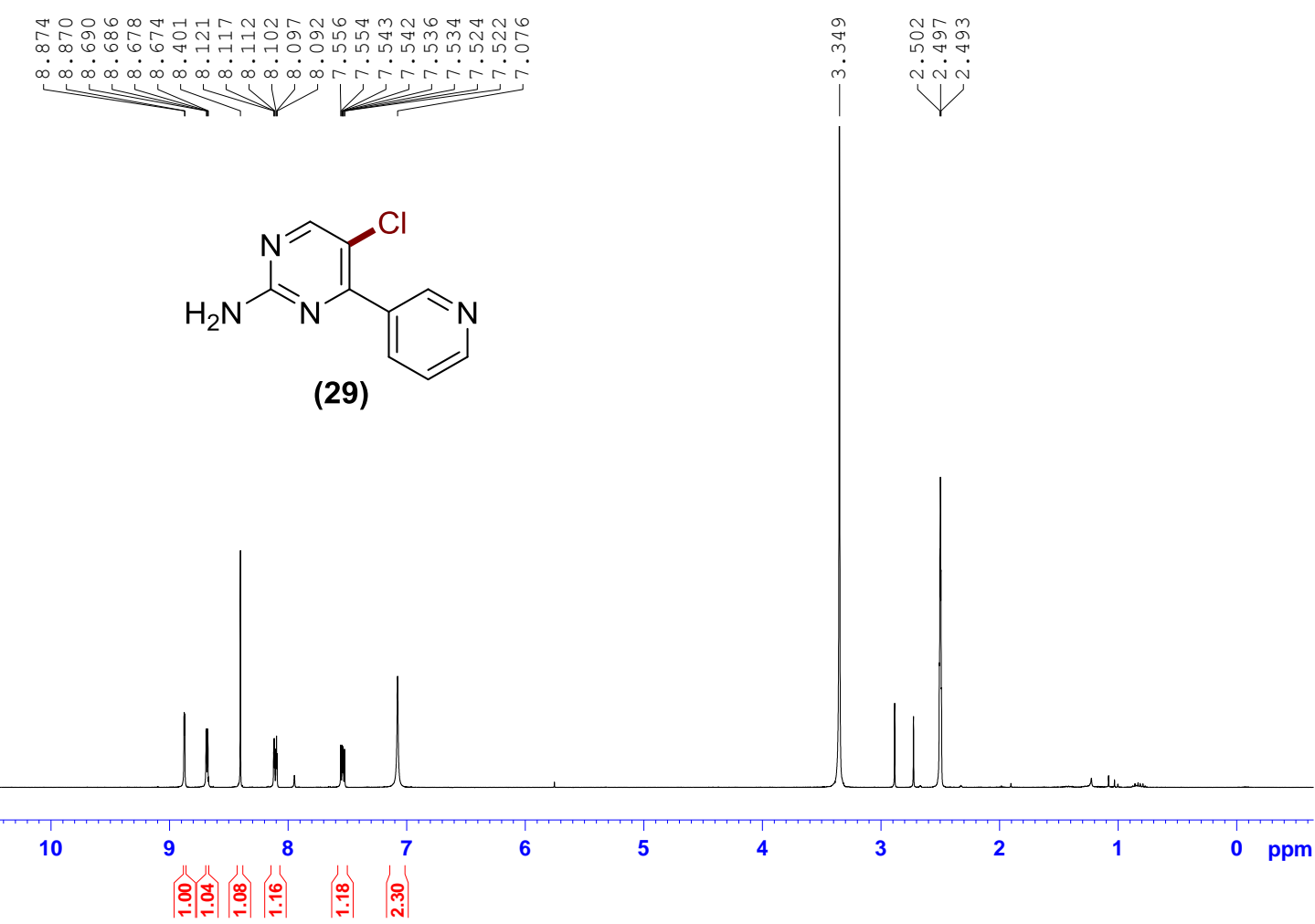

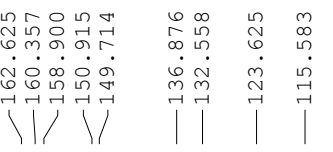

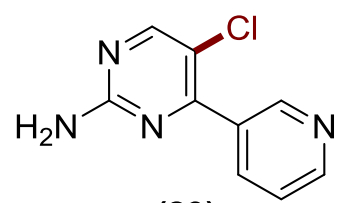

(29)

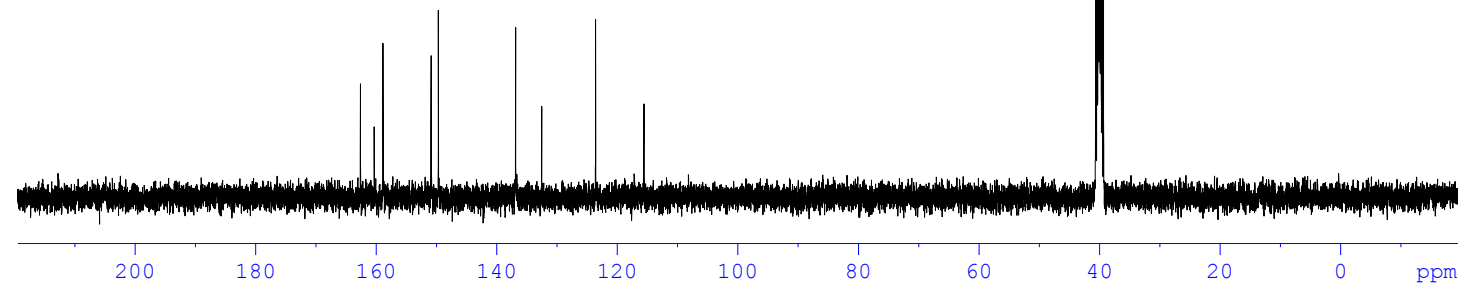




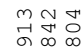

$$
\begin{aligned}
& \text { लंखिं }
\end{aligned}
$$
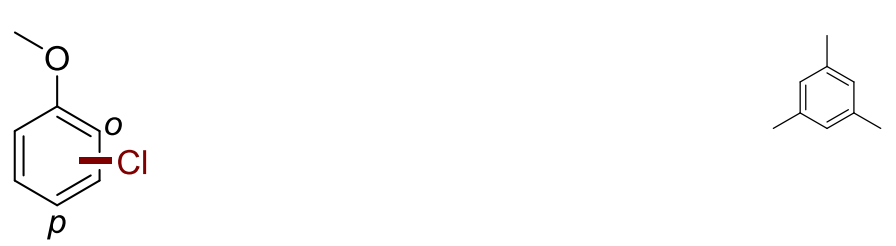

(30)

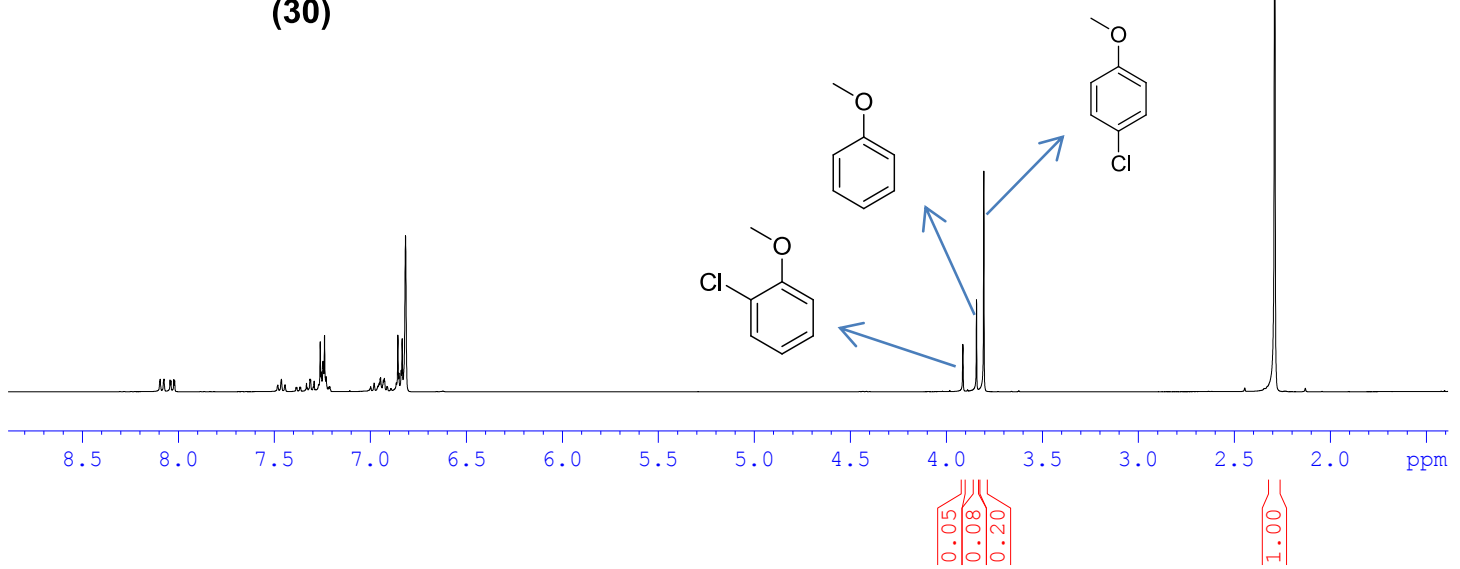




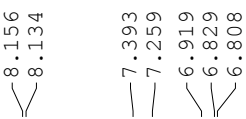

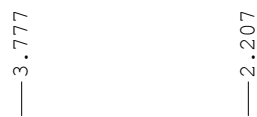<smiles>COc1ccc(NC(C)=O)c(Cl)c1</smiles>

(31)

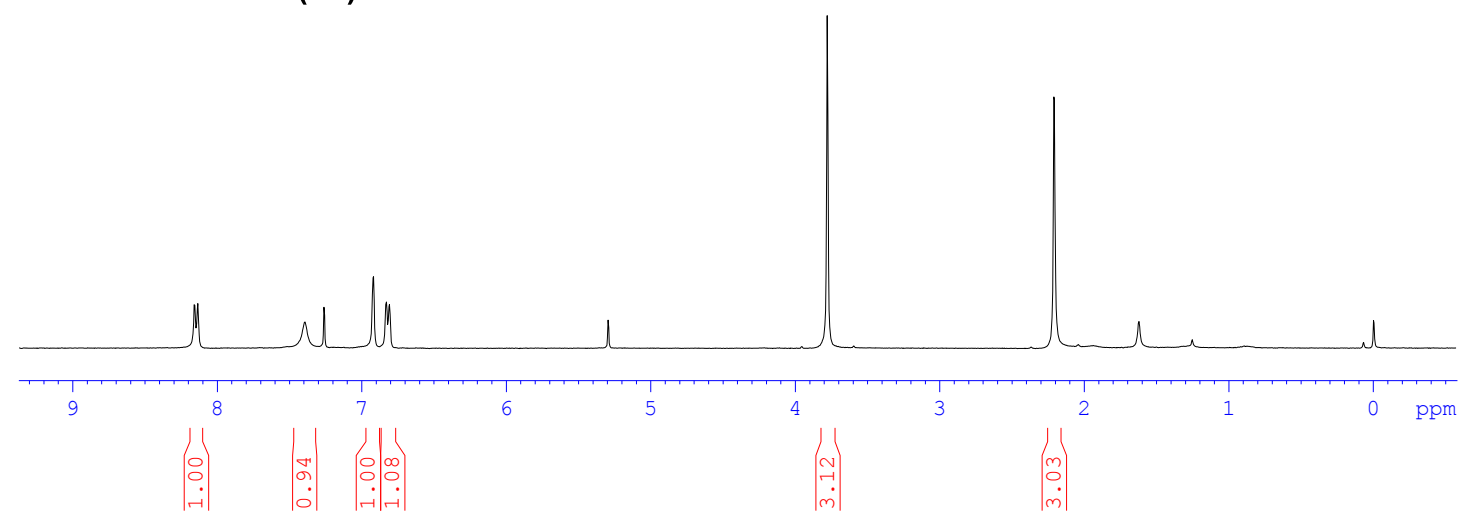

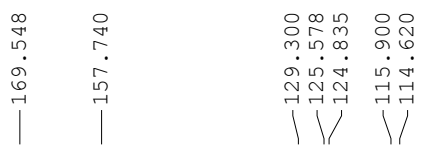<smiles>COc1ccc(NC(C)=O)c(Cl)c1</smiles>

(31)

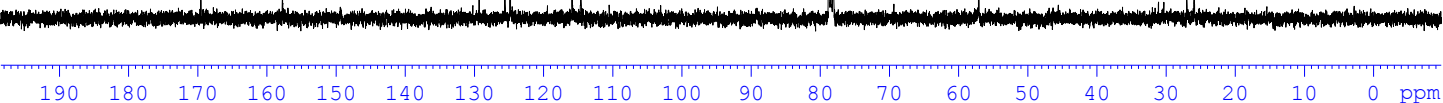




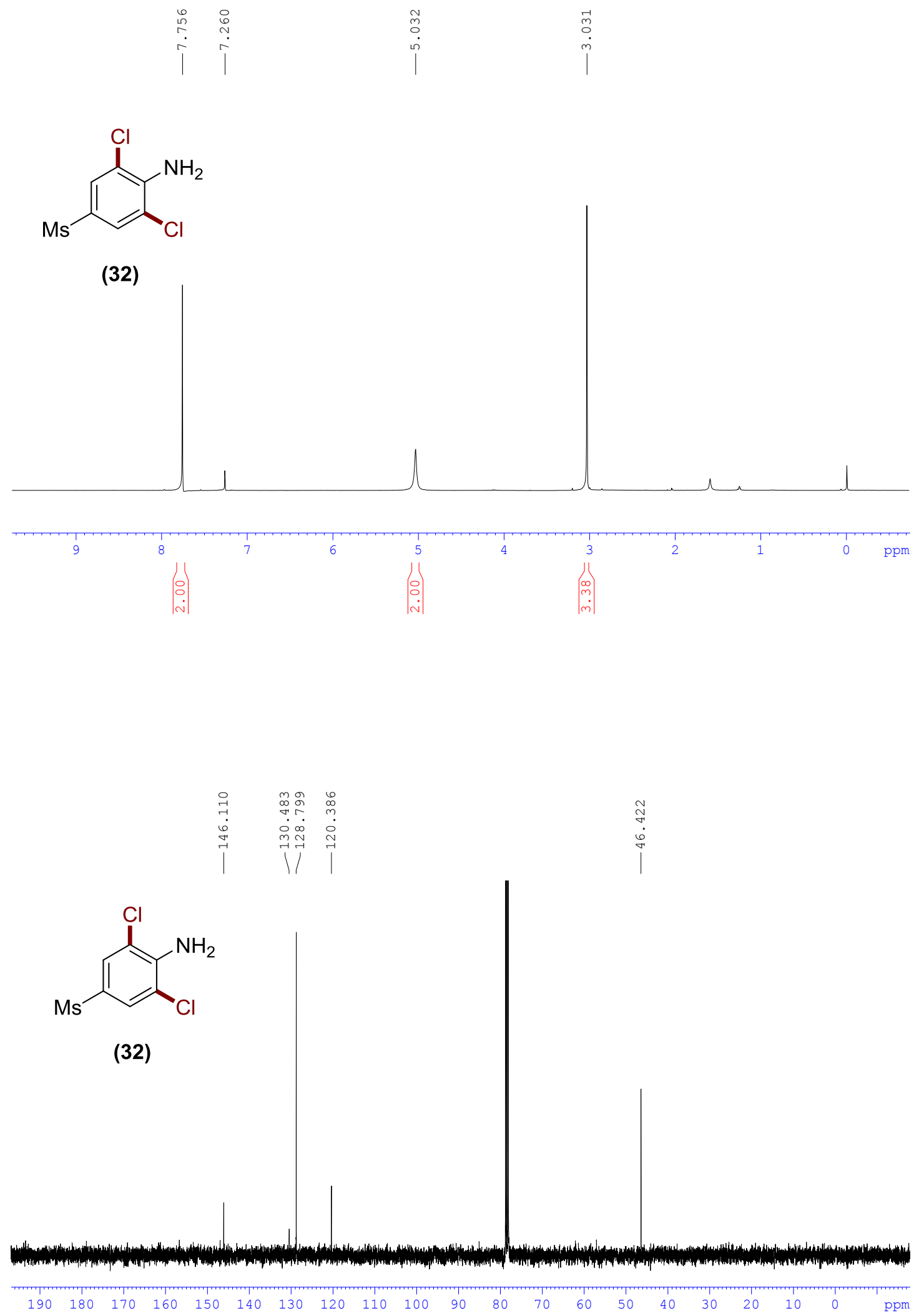


<smiles>Nc1c(Cl)cc(Cl)cc1Cl</smiles>

(33)
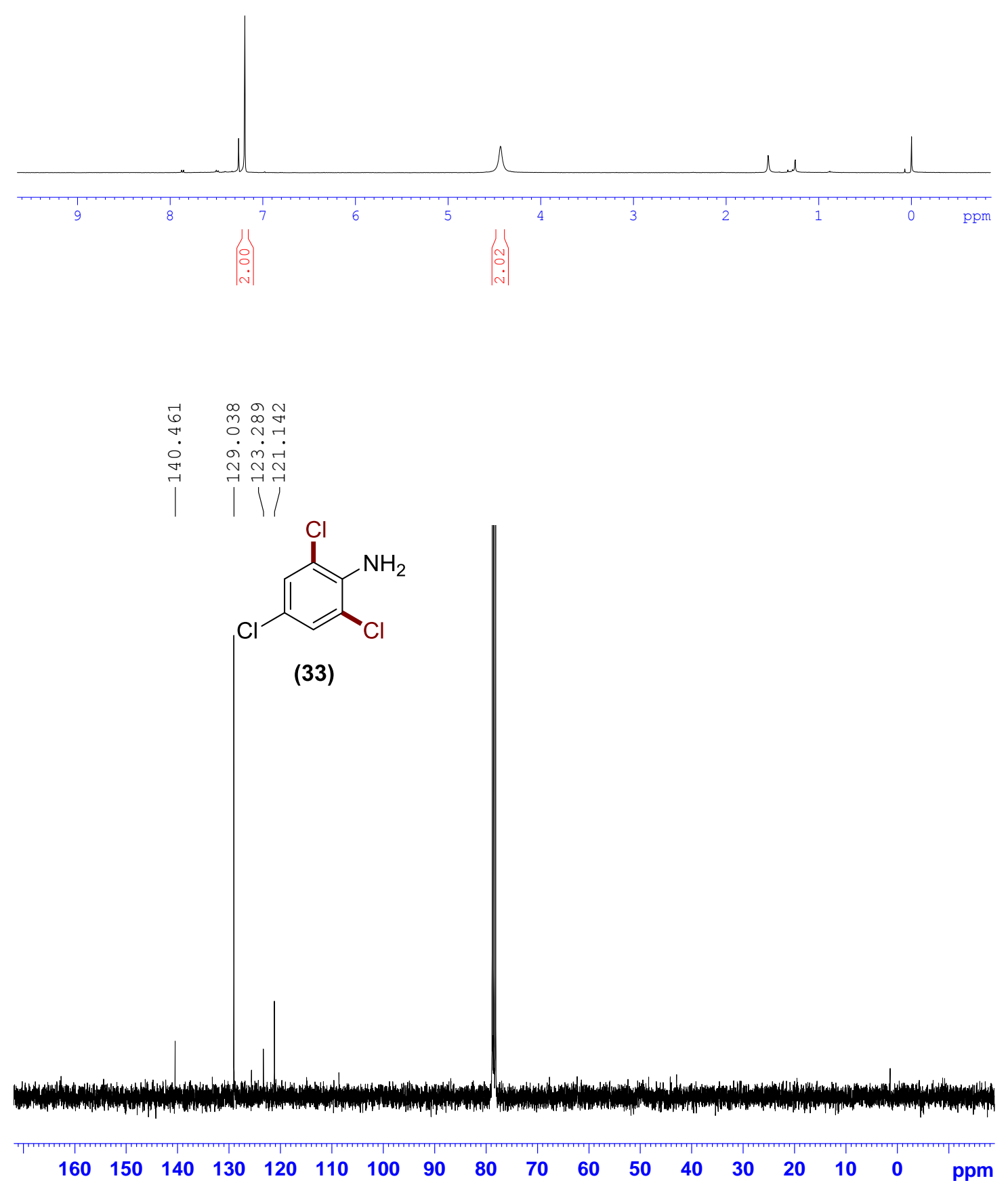


$$
\text { ํํำ }
$$<smiles>Nc1c(Cl)cc(Cl)c(Cl)c1Cl</smiles>

(34)
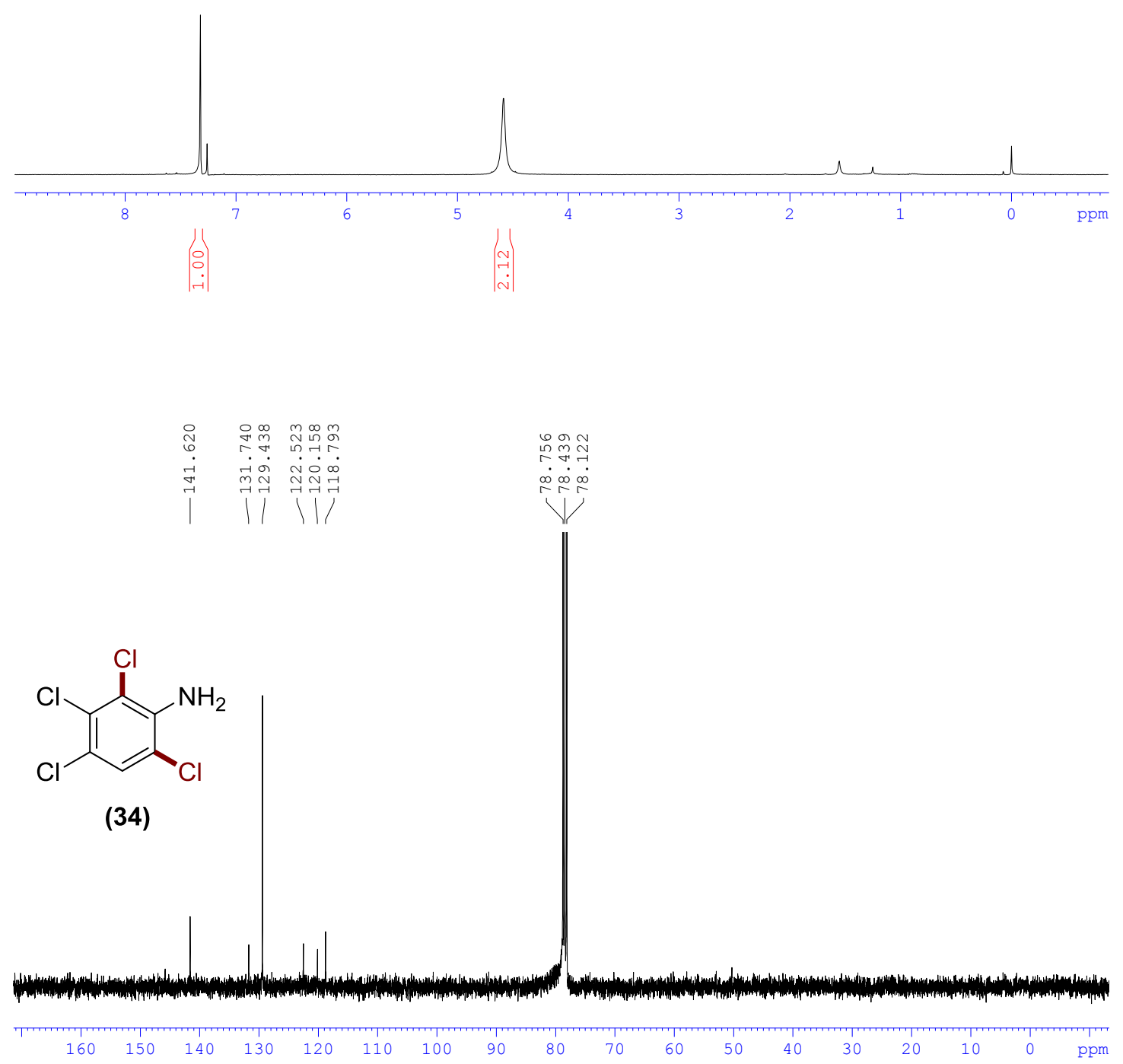


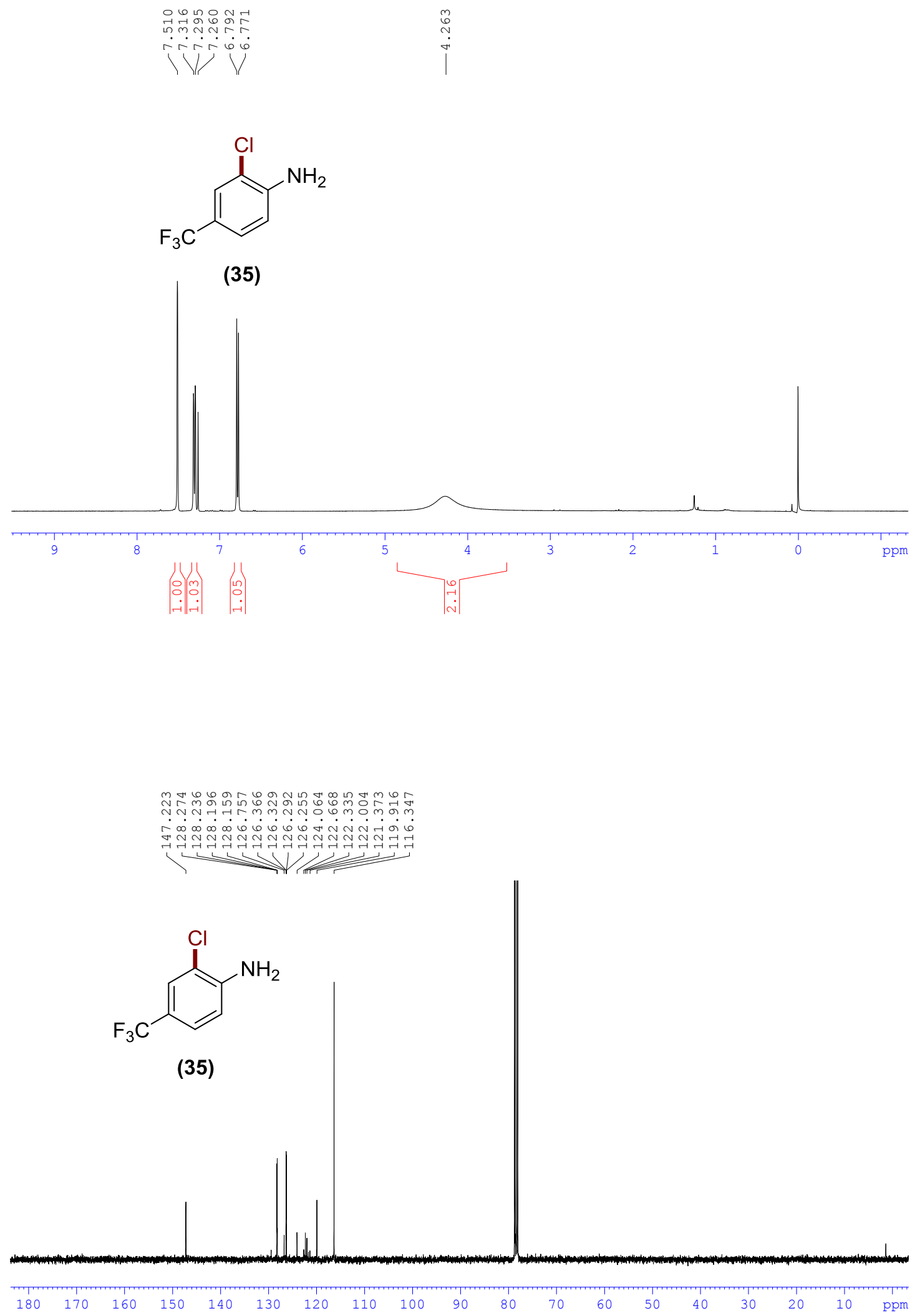




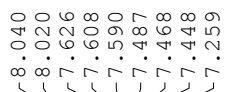

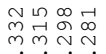

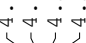<smiles>CCOC(=O)C(Cl)(Cl)C(=O)c1ccccc1</smiles>

(36)
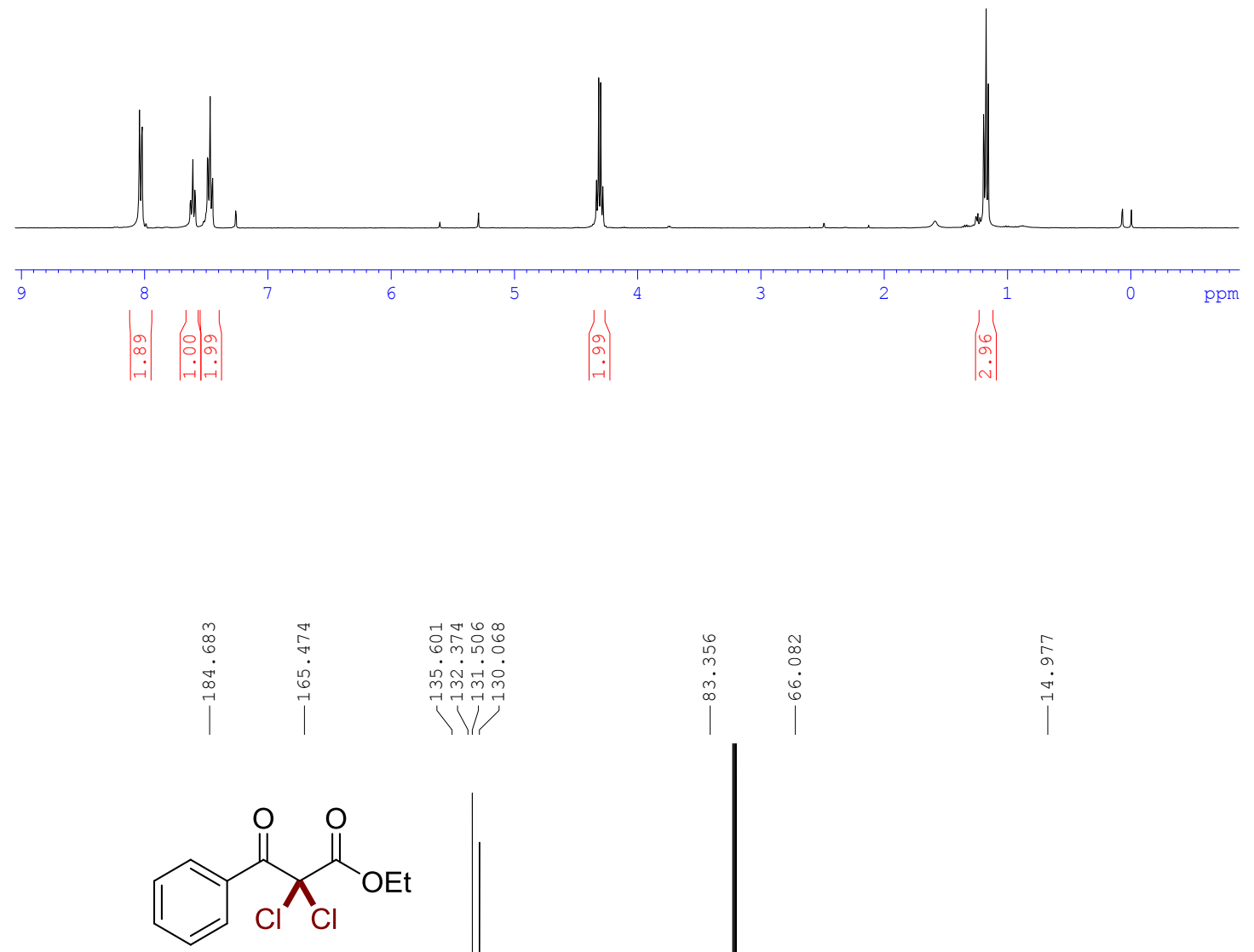

(36)

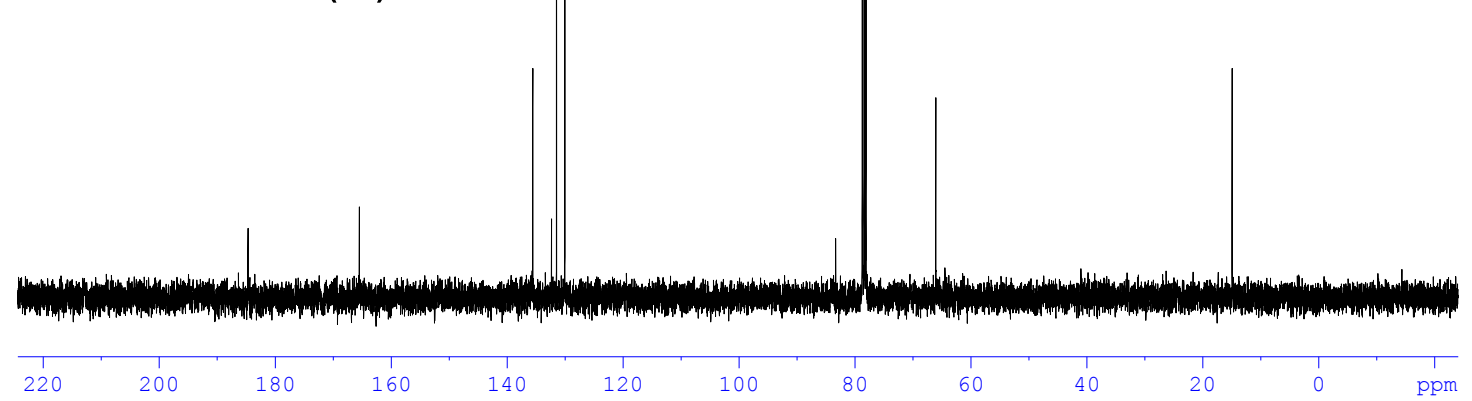




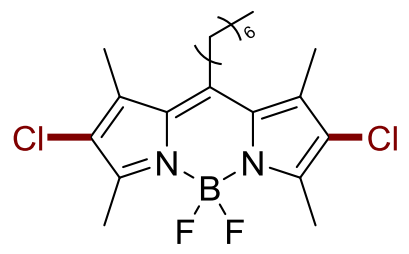

(37)
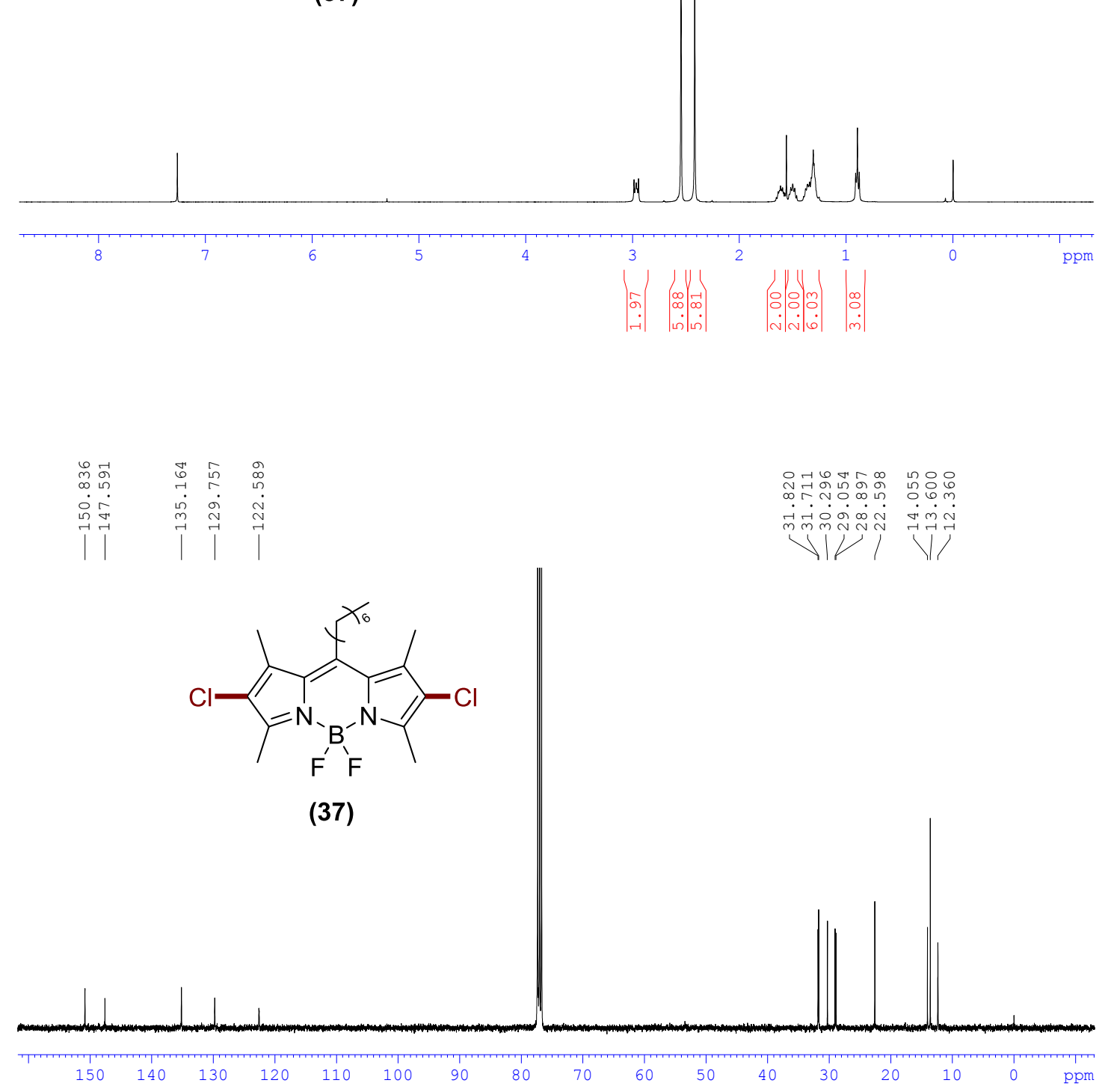


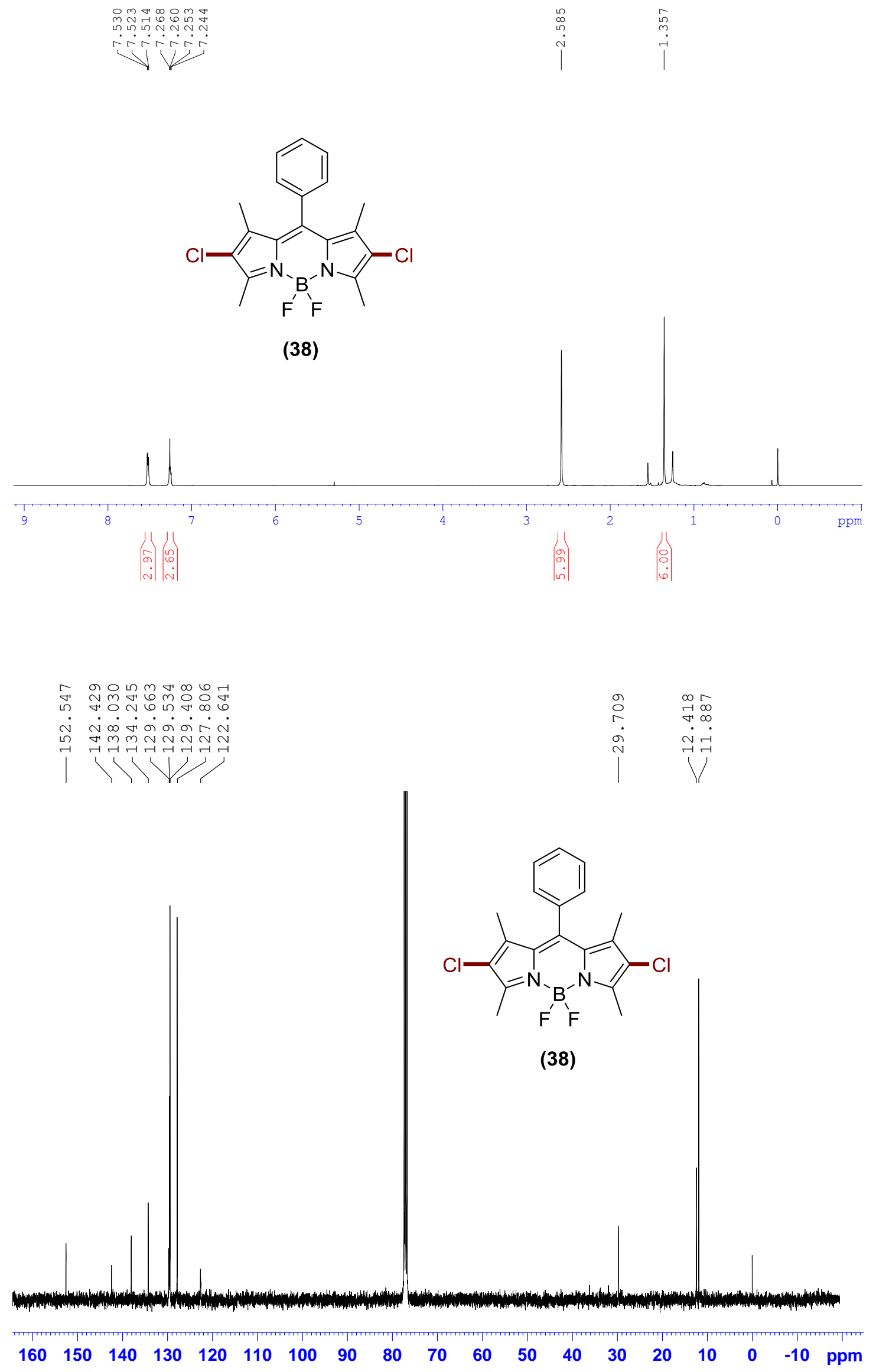



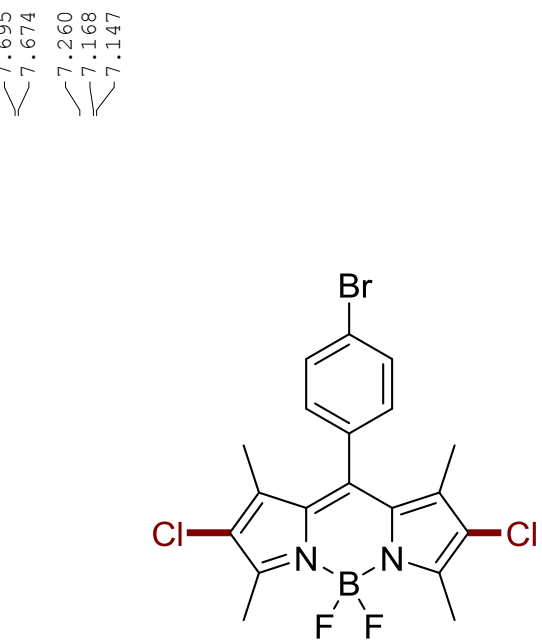

(39)
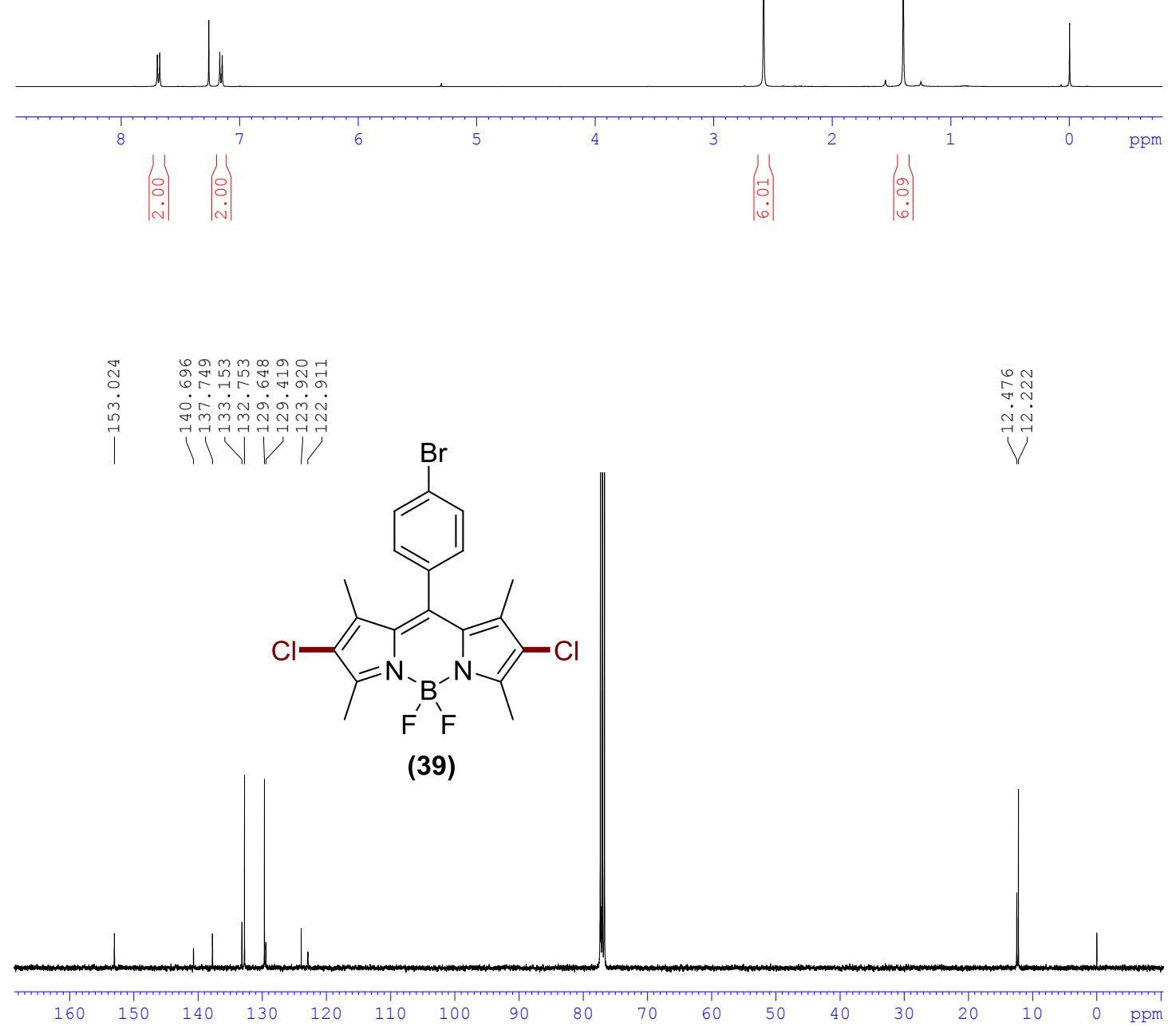


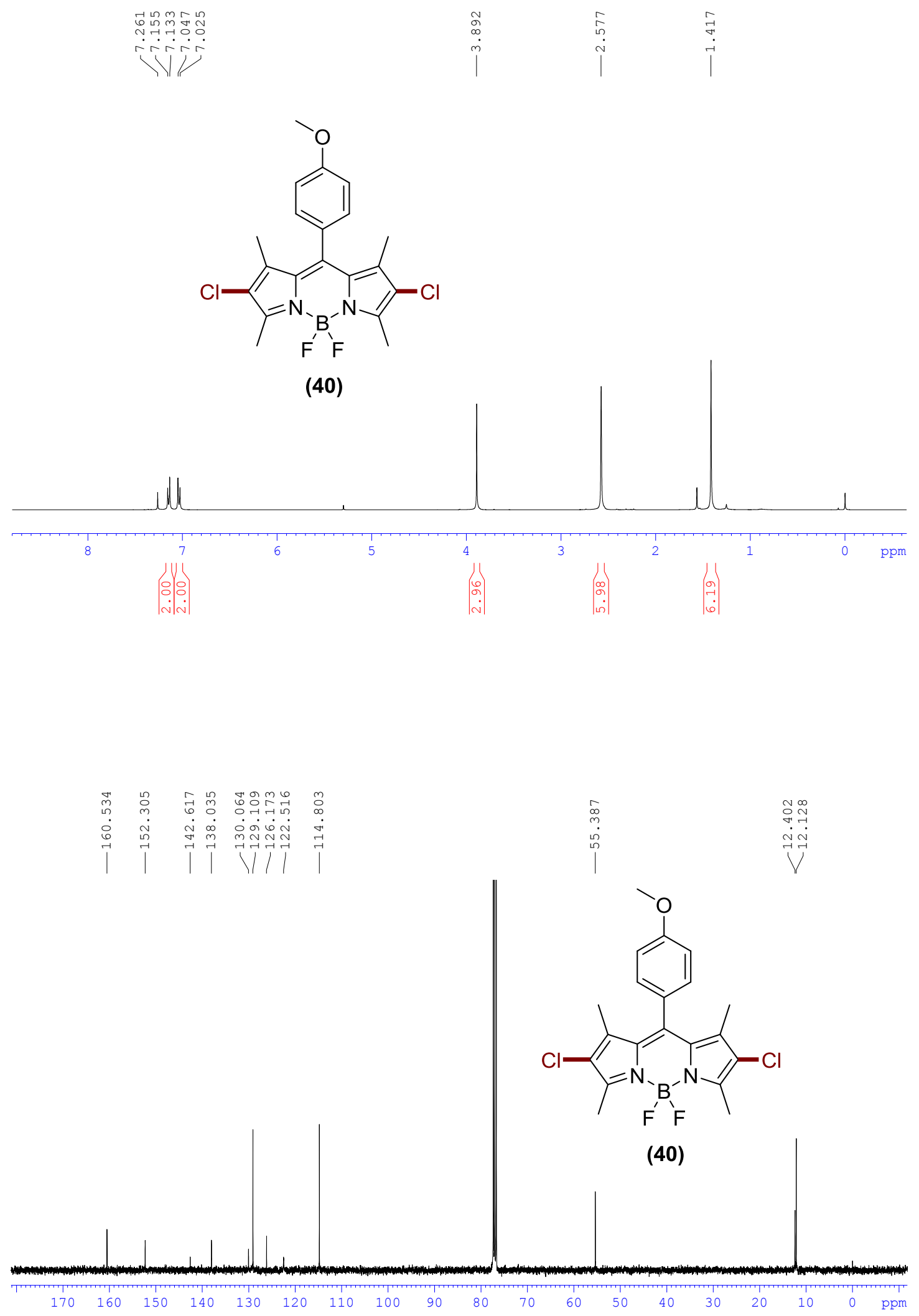


<smiles></smiles>

(41)
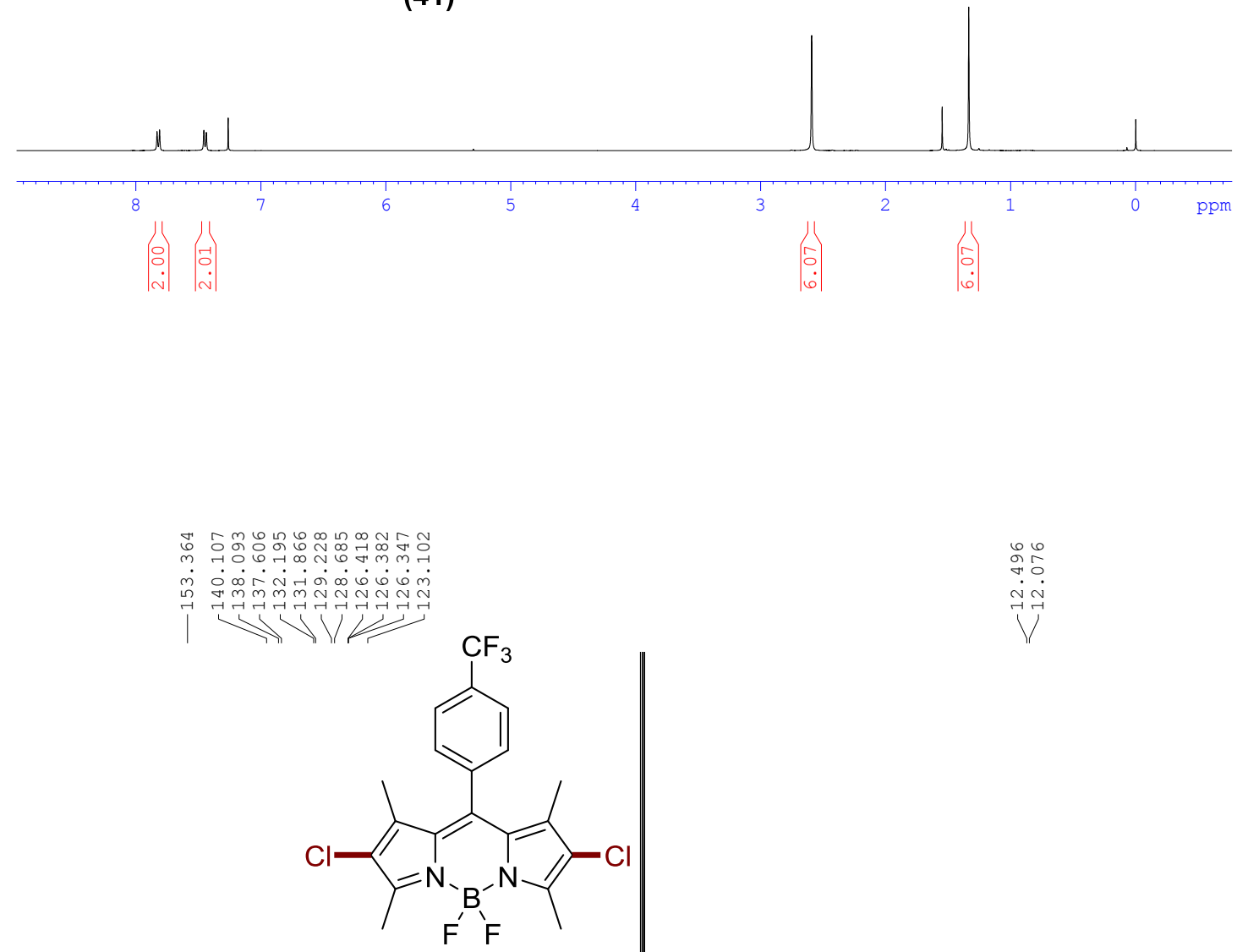

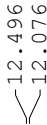

(41)

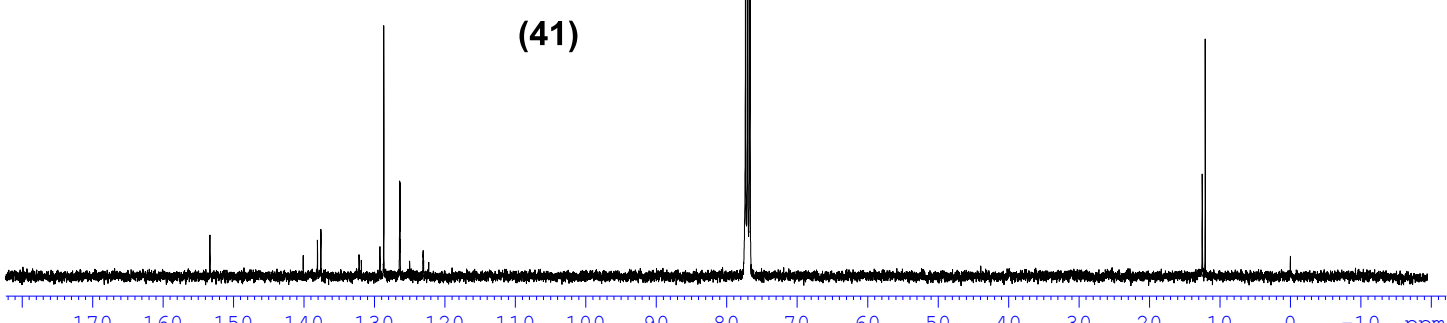




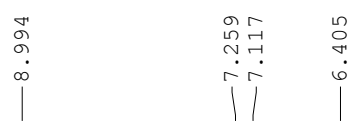

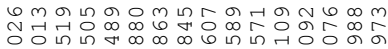

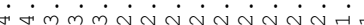

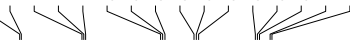<smiles>O=C1CCc2cc(Cl)c(OCCCCBr)cc2N1</smiles>

(43)
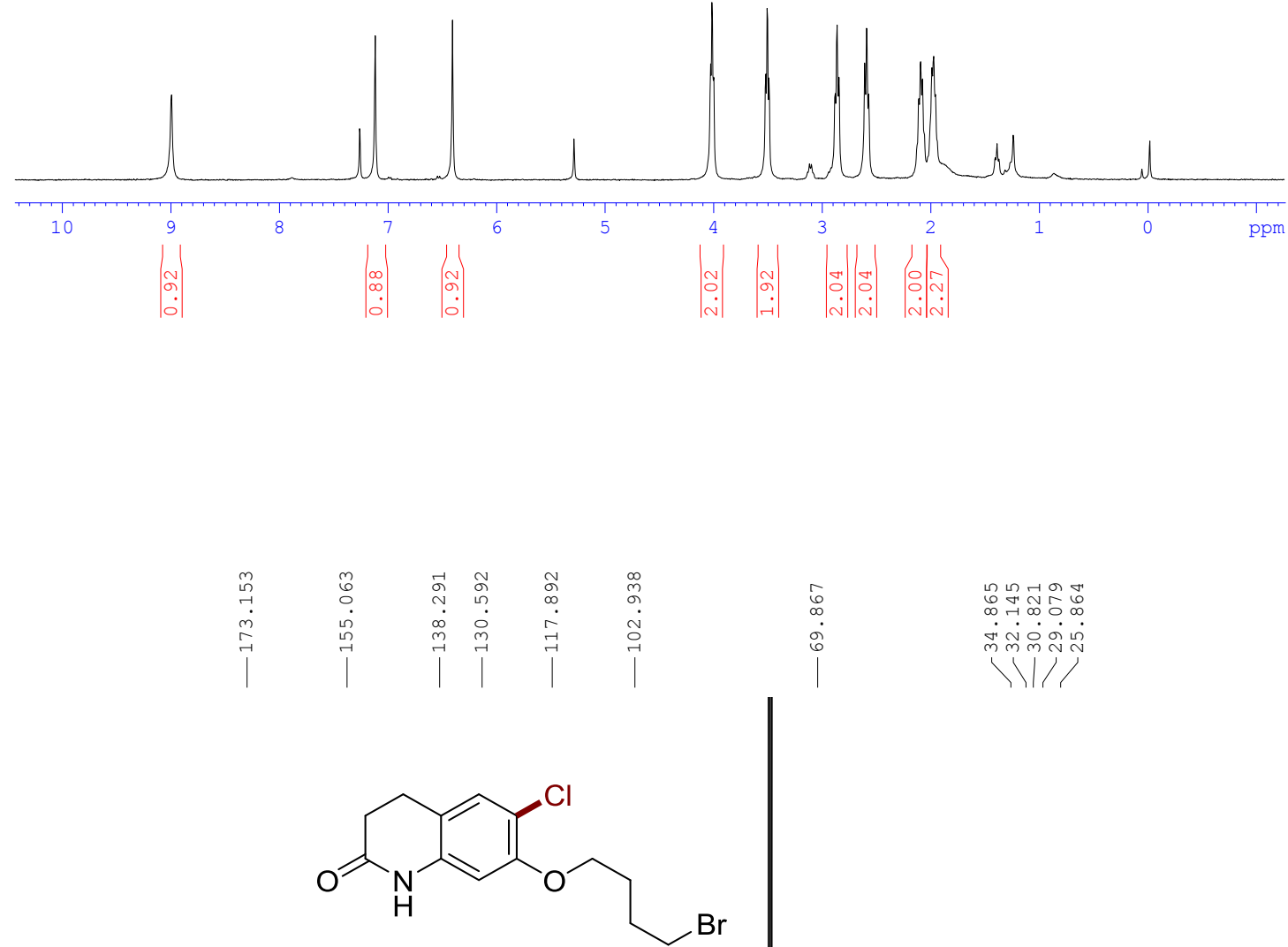

(43)

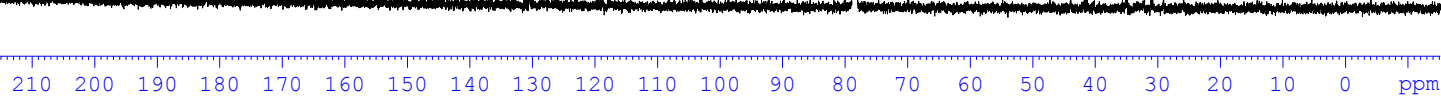




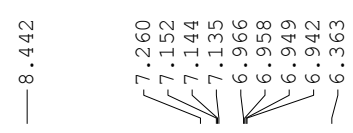

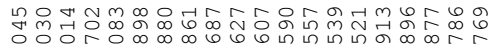

作

V 1 Wh<smiles>O=C1CCc2cc(Cl)c(OCCCCN3CCN(c4cccc(Cl)c4Cl)CC3)cc2N1</smiles>

(45)
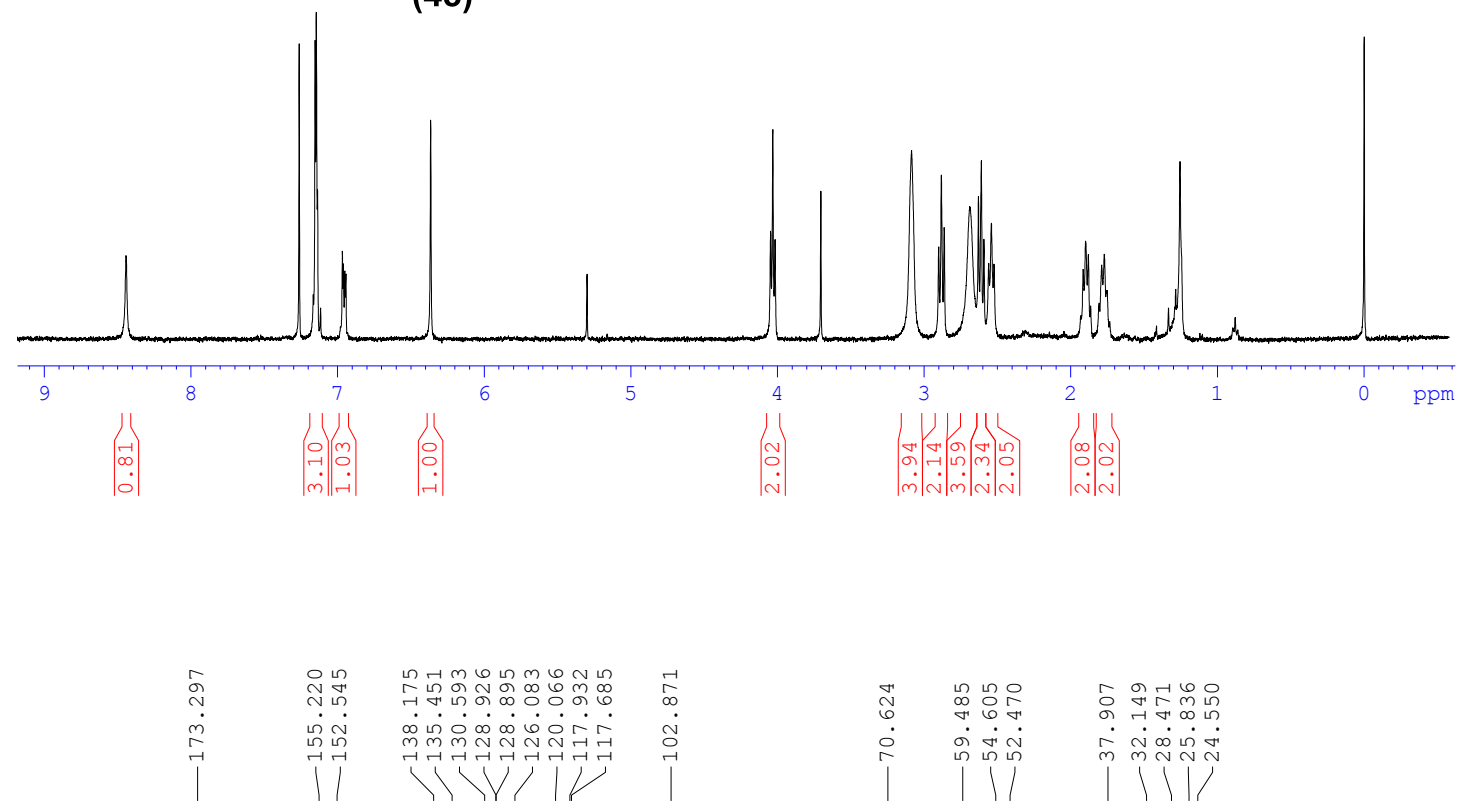<smiles>O=C1CCc2cc(Cl)c(OCCCCN3CCN(c4cccc(Cl)c4Cl)CC3)cc2N1</smiles>

(45)

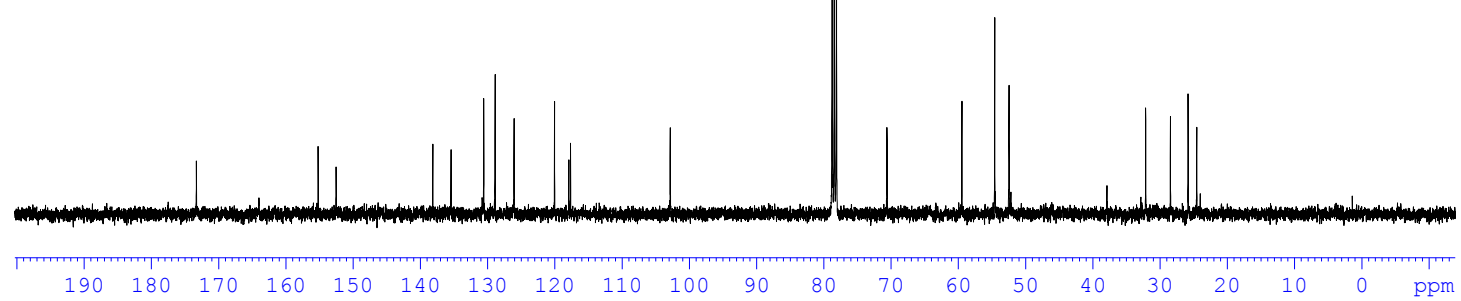

